Draft version August 6, 2018

Typeset using $\mathrm{LAT}_{\mathrm{E}} \mathrm{X}$ preprint style in AASTeX61

\title{
THE MASSIVE STAR-FORMING REGIONS OMNIBUS X-RAY CATALOG, SECOND INSTALLMENT
}

\author{
Leisa K. Townsley ${ }^{1}$ Patrick S. Broos,${ }^{1}$ Gordon P. Garmire, ${ }^{2}$ Gemma E. Anderson ${ }^{3}$ \\ Eric D. Feigelson, ${ }^{1}$ Tim Naylor, ${ }^{4}$ and Matthew S. Povich ${ }^{5}$
}

\footnotetext{
${ }^{1}$ Department of Astronomy $\&$ Astrophysics, 525 Davey Laboratory, Pennsylvania State University, University Park, PA 16802, USA

${ }^{2}$ Huntingdon Institute for X-ray Astronomy, LLC, 10677 Franks Road, Huntingdon, PA 16652, USA

${ }^{3}$ International Centre for Radio Astronomy Research, Curtin University, GPO Box U1987, Perth, WA 6845, Australia ${ }^{4}$ School of Physics, University of Exeter, Stocker Road, Exeter EX4 4QL, UK

${ }^{5}$ Department of Physics and Astronomy, California State Polytechnic University, 3801 West Temple Ave, Pomona, CA 91768, USA
}

(Accepted 2 Feb 2018, ApJS)

\begin{abstract}
We present the second installment of the Massive Star-forming Regions (MSFRs) Omnibus X-ray Catalog (MOXC2), a compilation of X-ray point sources detected in Chandra/ACIS observations of 16 Galactic MSFRs and surrounding fields. MOXC2 includes 13 ACIS mosaics, three containing a pair of unrelated MSFRs at different distances, with a total catalog of 18,396 point sources. The MSFRs sampled range over distances of $1.3 \mathrm{kpc}$ to $6 \mathrm{kpc}$ and populations varying from single massive protostars to the most massive Young Massive Cluster known in the Galaxy. By carefully detecting and removing X-ray point sources down to the faintest statistically-significant limit, we facilitate the study of the remaining unresolved X-ray emission. Through comparison with mid-infrared images that trace photon-dominated regions and ionization fronts, we see that the unresolved X-ray emission is due primarily to hot plasmas threading these MSFRs, the result of feedback from the winds and supernovae of massive stars. The 16 MSFRs studied in MOXC2 more than double the MOXC1 sample, broadening the parameter space of ACIS MSFR explorations and expanding Chandra's substantial contribution to contemporary star formation science.
\end{abstract}

Keywords: X-Rays: stars — stars: early-type — stars: formation — Hiı regions

townsley@astro.psu.edu,patrick.broos@icloud.com 
TOWNSLEY ET AL.

\section{INTRODUCTION}

To explore basic questions of Galactic star formation and structure, the first step is to identify the stars that make up Massive Star-Forming Regions (MSFRs), where most massive stars form (Motte et al. 2017). This simple goal is surprisingly hard to achieve; MSFRs are typically far away, behind large absorbing columns in the Galactic Plane. Infrared (IR) surveys are sensitivity-limited by bright extended emission and confusion-limited at longer wavelengths; only IR-excess stars found outside the brightest cluster cores can be reliably catalogued. This problem is not remedied as massive clusters age; by the time the natal dust and gas in a giant HiI region have been dispersed by massive star feedback, their clusters have expanded in size and their pre-main sequence (pre-MS) stars have dissipated their disks (and their IR excesses), so they are lost in the overwhelming contamination of unrelated field stars.

Ushering in the 21st century, the Chandra X-ray Observatory brought high spatial resolution to Xray astronomy, opening a new window into the workings of MSFRs. Chandra observations, combined with IR surveys, provide the best stellar census available for MSFRs. This is because Chandra has a large field of view that often captures an entire MSFR in a single pointing, it has sub-arcsecond spatial resolution on-axis, the instrumental background is low, and pre-MS stars are strong X-ray emitters independent of their disk status. Even the faintest X-ray sources that are matched to IR sources usually indicate MSFR membership. Additionally, Chandra observations do not suffer the bright, highly-variable backgrounds that plague IR studies.

Because of its fine spatial resolution and hard X-ray spectral response, Chandra is good at finding massive stars and intermediate-mass pre-MS stars (IMPS) as well as lower-mass pre-MS stars. Once all of these X-ray point sources are identified and removed from Chandra images, it is possible to use the remaining unresolved Chandra emission to map the hot, shocked interstellar medium (ISM) created by massive star winds and supernova feedback. This diffuse X-ray emission fills HiI region bubbles and permeates superbubbles; these structures trace the framework of star formation in the Galaxy and in turn define starburst clusters in other galaxies. Chandra has established definitively that star formation proceeds in the presence of 1-10 million degree plasmas (Townsley et al. 2003) and that even the youngest massive stars, still ionizing hyper- and ultra-compact HiI regions (UCHirRs), blast their cold, molecular birth environments with hard X-rays (Anderson et al. 2011). Thus no picture of a MSFR's evolution and environmental impact is complete without the Chandra perspective.

Chandra has amassed a large body of MSFR observations, giving new insights into the stellar populations and energetics of individual regions and enabling ambitious multi-target comparison projects. Examples focusing on individual MSFR complexes include The Astrophysical Journal Supplement Series special issues on the Chandra Orion Ultradeep Project (COUP) (Volume 160 Number 2, e.g., Getman et al. 2005) observations of the Orion Nebula Cluster and the Chandra Carina Complex Project (CCCP) (Volume 194 Number 1, e.g., Townsley et al. 2011a) observations of the Great Nebula in Carina. We look forward to the Chandra Cygnus OB2 Legacy Survey (Wright et al. 2014). Multi-target comparisons include the Massive Young Star-Forming Complex Study in Infrared and Xray (MYStIX, e.g., Feigelson et al. 2013) and the Star Formation in Nearby Clouds project (SFiNCs, e.g., Getman et al. 2017).

The immediate precursor to the current work was the first installment of the MSFRs Omnibus Xray Catalog (MOXC1, Townsley et al. 2014) which presented Chandra point source lists and images 
of diffuse X-ray emission for 12 MSFRs (seven for MYStIX and five more distant regions). This paper (hereafter MOXC2) closely follows the structure of MOXC1 and does not repeat the methodologies and analysis details explained there, thus we refer readers to MOXC1 for more background on the MOXC2 effort. All of the Chandra data used for our MSFR studies comes from the Advanced CCD Imaging Spectrometer (ACIS) instrument (Garmire et al. 2003).

With MOXC2, we expand our analysis of Chandra/ACIS data to include off-axis CCDs and archival data on fields adjacent to our target MSFRs, which were observed for other reasons. These ancillary pointings often go well beyond most cluster radii and provide the Galactic context for our MSFRs, informing our efforts to understand the ecology of large-scale star formation and feedback. In particular, these wide-field ACIS mosaics create new opportunities to understand distributed populations of young stars, multi-epoch star formation, and the extent of hot plasmas surrounding massive molecular filaments and threading giant molecular clouds (GMCs). The influence of evolving MSFRs extends over 10-100 pc scales and includes supernova remnants (SNRs), pulsars and pulsar wind nebulae (PWNe), gamma-ray sources, X-ray binaries, and diffuse plasmas from massive star feedback (both winds and supernovae). We are using these new wide-field X-ray analysis capabilities to improve our understanding of the energetics, populations, and environments of GMCs, their networks of massive filaments, and their multiple MSFRs, and how they drive Galactic evolution.

The MSFR targets featured in this paper are shown in Table 1. As in MOXC1, we include a rough limiting luminosity $L_{t c}$ and the corresponding limiting mass $M_{50 \%}$ where the brighter half of the $\mathrm{X}$-ray population is sampled, based on COUP results (Preibisch et al. 2005). This $L_{t c}$ comes from a PIMMS $S^{1}$ calculation for a 5-count detection on-axis, for a source with an apec thermal plasma with $k T=2.7 \mathrm{keV}$ and abundance $0.4^{*} \mathrm{Z}_{\odot}$. These values are typical for a pre-MS star (Preibisch et al. 2005).

We have assembled and analyzed 13 wide-field ACIS mosaics for MOXC2, amassing a catalog of 18,396 X-ray point sources and adaptively-smoothed images of the remaining unresolved X-ray emission in each mosaic. In three of these regions, MOXC2 exploits chance superpositions of unrelated MSFRs that just happen to be superposed on the sky; these are IRAS 19410+2336 and NGC 6823, W42 and RSGC1, and W33 and Cl 1813-178.

The number of X-ray point sources found for each target (Table 1 Column 10) varies substantially between targets. This is due to a combination of field of view and sensitivity; short observations and/or single pointings naturally result in fewer source detections than deeper observations and/or wide-field mosaics. Additionally, the MOXC2 sample of MSFRs includes a wide variety of contexts in which we find massive stars, from sparse clumps containing just a handful of young stars to Young Massive Clusters (YMCs) containing tens of thousands of members.

\section{CHANDRA OBSERVATIONS AND DATA ANALYSIS}

\subsection{Observations}

The Chandra/ACIS observations used for MOXC2 are summarized in Table 2 and are identified by a unique Observation Identification (ObsID) number. All of these datasets are available in the Chandra archive. Observations are ordered by date for each target; target names as used in this

${ }^{1}$ http://asc.harvard.edu/toolkit/pimms.jsp 
TOWNSLEY ET AL.

Table 1. MOXC2 Targets

\begin{tabular}{|c|c|c|c|c|c|c|c|c|c|c|}
\hline $\begin{array}{r}\text { MOXC2 } \\
\text { Target }\end{array}$ & $\begin{array}{c}\text { Galactic } \\
(l, b)\end{array}$ & $\begin{array}{c}\text { Celestial J2000 } \\
(R A, D e c)\end{array}$ & $\begin{array}{c}\text { Distance } \\
(\mathrm{kpc})\end{array}$ & $\begin{array}{c}\text { Scale } \\
(\operatorname{arcmin} / \mathrm{pc})\end{array}$ & $\begin{array}{l}\left\langle A_{V}\right\rangle \\
\text { (mag) }\end{array}$ & $\begin{array}{l}\text { Nominal } \\
\text { Exp (ks) }\end{array}$ & $\begin{array}{l}\log L_{t c} \\
(\mathrm{erg} / \mathrm{s})\end{array}$ & $\begin{array}{l}M_{50 \%} \\
\left(M_{\odot}\right)\end{array}$ & $\begin{array}{c}\text { X-ray Srcs } \\
(\#)\end{array}$ & Distance Reference \\
\hline (1) & (2) & (3) & (4) & (5) & (6) & (7) & (8) & (9) & (10) & (11) \\
\hline NGC 6334 & $350.99+0.62$ & $171947.1-360835$ & 1.3 & 2.64 & 4 & 40 & 29.64 & 0.3 & 4674 & Wu et al. (2014); Chibueze et al. (2014) \\
\hline W75N & $81.89+0.79$ & $203836.5+423846$ & 1.30 & 2.64 & 50 & 30 & 30.53 & 1.2 & 356 & Rygl et al. (2012) \\
\hline $\mathrm{RCW} 120$ & $348.22+0.46$ & $171220.8-382931$ & 1.34 & 2.57 & 40 & 79 & 30.08 & 0.6 & 1692 & Zavagno et al. (2007) \\
\hline IRAS $20126+4104$ & $78.13+3.62$ & $201429.1+411333$ & 1.64 & 2.10 & 70 & 39 & 30.79 & 1.8 & 493 & Moscadelli et al. (2011) \\
\hline W31N & $10.32-0.15$ & $\begin{array}{llllll}18 & 08 & 59.1 & -20 & 05 & 08\end{array}$ & 1.75 & 1.96 & 18 & 39 & 30.36 & 1.0 & 974 & Deharveng et al. (2015) \\
\hline IRAS $19410+2336$ & $59.78+0.06$ & $194311.4+234406$ & 2.16 & 1.59 & 20 & 9 & 31.14 & bright & 411 & Xu et al. (2009) \\
\hline $\mathrm{W} 42$ & $25.36-0.19$ & $1838 \quad 14.6-064919$ & 2.2 & 1.56 & 11 & 54 & 30.27 & 0.8 & 840 & Blum et al. (2000) \\
\hline NGC 6823 & $59.40-0.15$ & $194311.0+231745$ & 2.3 & 1.49 & 3 & 8 & 30.63 & 1.3 & see IRAS 19410 & Massey et al. (1995) \\
\hline W33 & $12.81-0.20$ & $\begin{array}{llllll}18 & 14 & 13.5 & -17 & 55 & 42\end{array}$ & 2.4 & 1.43 & 20 & 38 & 30.68 & 1.5 & 1960 & Immer et al. (2013) \\
\hline NGC 7538 & $111.54+0.81$ & $231345.5+612817$ & 2.65 & 1.32 & 11 & 29 & 30.66 & 1.4 & 487 & Moscadelli et al. (2009) \\
\hline G333 & $333.03-0.44$ & 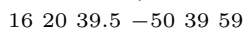 & 2.6 & 1.32 & 15 & 60 & 30.44 & 1.1 & 3935 & Figuerêdo et al. (2005) \\
\hline AFGL 2591 & $78.89+0.71$ & $202924.9+401121$ & 3.33 & 1.03 & 100 & 30 & 31.66 & bright & 288 & Rygl et al. (2012) \\
\hline $\mathrm{G} 34.4+0.23$ & $34.40+0.23$ & $1853 \quad 18.5+012448$ & 3.7 & 0.93 & 200 & 63 & 31.79 & bright & 565 & Rathborne et al. (2005) \\
\hline Cl $1813-178$ & $12.74-0.01$ & $181324.3-175331$ & 3.8 & 0.90 & 9 & 59 & 30.65 & 1.4 & see W33 & Messineo et al. (2011) \\
\hline $\mathrm{Wd} 1$ & $339.55-0.40$ & $164704.0-455105$ & 4.0 & 0.86 & 11 & 55 & 30.69 & 1.5 & 1721 & Gennaro et al. (2011) \\
\hline RSGC1 & $25.27-0.16$ & $183758.0-065300$ & 6 & 0.57 & 23 & 74 & 31.24 & bright & see W42 & Froebrich \& Scholz (2013) \\
\hline
\end{tabular}

Note- Col. (5): Image scale assuming the distance given in Col. (4).

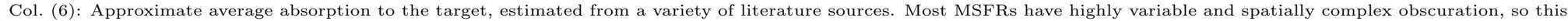
value should be used only as a rough indicator.

Col. (7): A typical exposure time for the main MSFRs. Most mosaics have a wide range of exposures; detailed exposure maps are shown in Section 4.

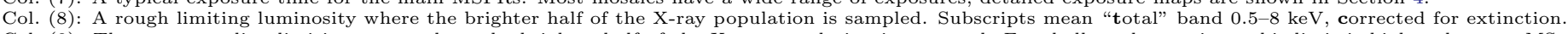

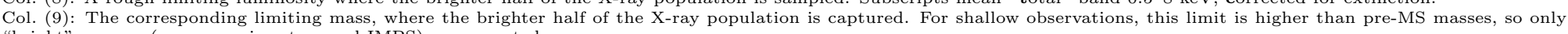
"bright" sources (some massive stars and IMPS) are expected.

Col. (10): Total number of X-ray sources found across the entire mosaic.

paper are given in Column (1) in bold, followed by the original name assigned by the study's principal investigator (PI), noted in Column (11). Those observations where Gordon Garmire is listed as PI came from the ACIS Instrument Team Guaranteed Time Observation (GTO) program; those listing Stephen Murray as PI came from the HRC Instrument Team GTO program. We note this fact because the Chandra GTO programs provided many of the original seed observations for the MSFRs studied here and for many other Galactic Plane observations that we use in our wide-field mosaics.

Virtually all of the ACIS data we have previously published were taken with the Observatory in the ACIS imaging array (ACIS-I) configuration (Garmire et al. 2003), with the optical axis near the center of a $2 \times 2$ array of $1024 \times 1024$-pixel CCDs covering roughly $17^{\prime} \times 17^{\prime}$ on the sky (with $0.492^{\prime \prime}$ pixels). Although such observations commonly include data from two CCDs lying far off-axis in the ACIS spectroscopy array (ACIS-S), we previously chose to discard data from those CCDs due to the poor angular resolution of the Chandra mirrors at large off-axis angles ${ }^{2}$.

In order to widen the field coverage around MSFRs and to include more archival data, MOXC2 includes the off-axis CCDs in ACIS-I observations and imaging observations taken in the ACIS-S configuration (Garmire et al. 2003), as shown in the MOXC2 exposure map mosaics in Section 4. Four of our MSFRs were observed with the ACIS-S imaging configuration. One side effect of this more liberal data selection policy is that most observations presented here include both front-side illuminated (FI) and back-side illuminated (BI) CCD detectors (Garmire et al. 2003). Because FI and BI detectors exhibit very different background spectra, their analysis must be done separately, thus we split each Chandra ObsID into separate FI and BI "virtual" observations within our data analysis workflow.

${ }^{2}$ See Figure 4.12 in the Chandra Proposers' Observatory Guide (http://asc.harvard.edu/proposer/POG/). 
Table 2. Log of Chandra Observations

\begin{tabular}{|c|c|c|c|c|c|c|c|c|c|c|c|}
\hline \multirow{2}{*}{$\begin{array}{c}\text { Target } \\
(1) \\
\end{array}$} & \multirow{2}{*}{$\begin{array}{c}\text { ObsID } \\
(2) \\
\end{array}$} & \multirow{2}{*}{$\begin{array}{c}\text { Start Time } \\
\text { (UT) } \\
(3) \\
\end{array}$} & \multirow{2}{*}{$\begin{array}{c}\text { Exposure }^{\mathrm{a}} \\
(\mathrm{s}) \\
(4) \\
\end{array}$} & \multicolumn{3}{|c|}{ Aimpoint $^{\mathrm{b}}$} & \multirow{2}{*}{$\begin{array}{l}\text { Roll } \\
\left({ }^{\circ}\right) \\
(8) \\
\end{array}$} & \multirow{2}{*}{$\begin{array}{c}\text { Mode }^{\mathrm{C}} \\
(9) \\
\end{array}$} & \multirow{2}{*}{$\begin{array}{c}\text { Detectors }^{\mathrm{d}} \\
(10) \\
\end{array}$} & \multirow{2}{*}{$\begin{array}{l}\text { PI } \\
(11) \\
\end{array}$} & \multirow{2}{*}{$\begin{array}{c}\text { TGAIN }^{\mathrm{e}}{ }^{\mathrm{f}} \\
(12) \\
\end{array}$} \\
\hline & & & & $\begin{array}{c}\mathrm{CCD} \\
(5) \\
\end{array}$ & $\begin{array}{c}\alpha_{\mathrm{J} 2000} \\
(6)\end{array}$ & $\begin{array}{c}\delta_{\mathrm{J} 2000} \\
(7)\end{array}$ & & & & & \\
\hline \multicolumn{12}{|l|}{ NGC 6334} \\
\hline NGC 6334 REGION 1 & 2574 & 2002-08-31T12:49 & 39648 & I3 & $17: 20: 54.00$ & $-35: 47: 03.9$ & 269 & TE-F & 012367 & Yuichiro Ezoe & acisD2002-08-01t_gainN0006 \\
\hline NGC 6334 REGION 2 & 2573 & 2002-09-02Т00:12 & 25473 & I3 & $17: 20: 01.00$ & $-35: 56: 07.0$ & 268 & TE-F & 012367 & Yuichiro Ezoe & acisD2002-08-01t_gainN0006 \\
\hline G351.2+0.1 & 3844 & 2003-10-04T19:21 & 14773 & S3 & $17: 22: 24.70$ & $-36: 10: 59.9$ & 261 & TE-VF & 235678 & David Helfand & acisD2003-08-01t_gainN0006 \\
\hline $\mathrm{G} 351.2+0.1$ & 4591 & 2004-07-31Т00:30 & 38012 & S3 & $17: 22: 28.00$ & $-36: 10: 59.9$ & 278 & TE-VF & 235678 & Stephen Murray & acisD2004-05-01t_gainN0006 \\
\hline NGC 6334 & 13436 & 2011-07-13Т09:37 & 62810 & I3 & $17: 19: 47.10$ & $-36: 08: 35.0$ & 288 & TE-VF & 012367 & Scott Wolk & acisD2011-05-01t_gainN0006 \\
\hline NGC 6334 & 12382 & 2011-07-17T19:19 & 32045 & I3 & $17: 19: 47.10$ & $-36: 08: 35.0$ & 288 & TE-VF & 012367 & Scott Wolk & acisD2011-05-01t_gainN0006 \\
\hline GM 24 & 18876 & 2016-06-27T12:52 & 16744 & I3 & $17: 17: 06.90$ & $-36: 21: 27.2$ & 302 & TE-VF & 012367 & Leisa Townsley & acisD2016-02-01t_gain_biN0002 \\
\hline GM 24 & 18082 & 2016-06-30T16:45 & 22658 & I3 & $17: 17: 06.90$ & $-36: 21: 27.2$ & 302 & TE-VF & 012367 & Leisa Townsley & acisD2016-02-01t_gain_biN0002 \\
\hline G350.776+0.831 & 18081 & 2016-07-23T18:50 & 37566 & I3 & $17: 18: 19.30$ & $-36: 12: 09.6$ & 281 & TE-VF & 01237 & Leisa Townsley & acisD2016-02-01t_gain_biNO002 \\
\hline \multicolumn{12}{|l|}{ W75N } \\
\hline W75N & 8893 & 2008-02-02Т01:15 & 29740 & I3 & $20: 38: 36.49$ & $+42: 38: 46.2$ & 5 & TE-VF & 012367 & Gordon Garmire & acisD2008-02-01t_gainN0006 \\
\hline \multicolumn{12}{|l|}{ RCW 120} \\
\hline СТВ $37 \mathrm{~A}$ & 6721 & 2006-10-07Т05:13 & 19905 & I3 & $17: 14: 35.80$ & $-38: 31: 24.5$ & 259 & TE-VF & 0123 & Hiroshi Nakajima & acisD2006-08-01t_gainN0006 \\
\hline HESS J1713-381 & 6692 & 2007-02-02T11:55 & 24836 & I3 & $17: 14: 04.09$ & $-38: 11: 05.3$ & 96 & TE-VF & 012367 & James Hinton & acisD2007-02-01t_gainN0006 \\
\hline RCW 120 & 13621 & 2012-06-30T12:28 & 49117 & I3 & $17: 12: 20.80$ & $-38: 29: 30.5$ & 303 & TE-VF & 0123 & Konstantin Getman & acisD2012-05-01t_gainN0006 \\
\hline RCW120 & 13276 & 2013-02-11Т07:39 & 29688 & I3 & $17: 12: 20.80$ & $-38: 29: 30.5$ & 93 & TE-VF & 0123 & Gordon Garmire & acisD2013-02-01t_gainN0006 \\
\hline IRAS $20126+4104$ & & & & & & & & & & & \\
\hline IRAS $20126+4104$ & 3758 & 2003-03-13Т02:43 & 38761 & I3 & $20: 14: 29.05$ & $+41: 13: 32.4$ & 58 & TE-VF & 012367 & Peter Hofner & acisD2003-02-01t_gainN0006 \\
\hline W31N & & & & & & & & & & & \\
\hline SGR $1806-20$ & 1827 & 2000-07-24T18:22 & 4393 & S3 & $18: 08: 40.32$ & $-20: 24: 41.1$ & 265 & TE-F & 235678 & Gautam Vasisht & acisD2000-05-01t_gainN0006 \\
\hline SGR $1806-20$ & 6224 & 2005-02-09Т07:01 & 18620 & I3 & $18: 08: 39.29$ & $-20: 24: 38.9$ & 88 & TE-F & 012367 & Derek Fox & acisD2005-02-01t_gainN0006 \\
\hline AX J180816-2021 & 8151 & 2007-10-26Т07:15 & 2107 & S3 & $18: 08: 16.79$ & $-20: 21: 43.1$ & 272 & TE-F & 235678 & Stephen Murray & acisD2007-08-01t_gainN0006 \\
\hline AX J180857-2004 & 10518 & 2009-02-10Т08:00 & 6467 & S3 & $18: 08: 57.40$ & $-20: 04: 33.6$ & 88 & TE-F & 235678 & Bryan Gaensler & acisD2009-02-01t_gainN0006 \\
\hline G9.95-0.81 & 10713 & 2009-02-10T10:21 & 9914 & I3 & 18:10:41.09 & $-20: 43: 33.4$ & 88 & TE-VF & 012367 & Gordon Garmire & acisD2009-02-01t_gainN0006 \\
\hline W31 North & 18452 & 2016-08-14T05:31 & 39243 & I3 & 18:08:59.09 & $-20: 05: 08.1$ & 268 & TE-VF & 012367 & Gordon Garmire & acisD2016-02-01t_gain_biN0002 \\
\hline $\begin{array}{c}\text { IRAS } 19410+2336 \\
\text { NGC } 6823\end{array}$ & & & & & & & & & & & \\
\hline $19410+2336$ & 1868 & 2001-10-15T22:25 & 8679 & S3 & $19: 43: 11.40$ & $+23: 44: 05.9$ & 276 & TE-VF & 235678 & Henrik Beuther & acisD2001-08-01t_gainN0006 \\
\hline AX J194310+2318 & 10502 & 2009-02-26Т09:52 & 2054 & S3 & $19: 43: 10.30$ & $+23: 18: 50.4$ & 58 & TE-F & 235678 & Bryan Gaensler & acisD2009-02-01t_gainN0006 \\
\hline W42; RSGC1 & & & & & & & & & & & \\
\hline HESS J1837-069 & 6719 & 2006-08-19T16:30 & 19897 & I3 & $18: 37: 43.00$ & $-06: 54: 20.9$ & 259 & TE-VF & 012367 & Gerd Puehlhofer & acisD2006-08-01t_gainN0006 \\
\hline $\mathrm{W} 42$ & 16673 & 2016-02-13Т16:26 & 53865 & I3 & 18:38:14.59 & $-06: 49: 18.9$ & 78 & TE-VF & 0123 & Leisa Townsley & acisD2016-02-01t_gain_biN0002 \\
\hline W33; Cl 1813-178 & & & & & & & & & & & \\
\hline G12.7-0.0 & 6685 & 2006-09-15Т00:56 & 29568 & I3 & $18: 13: 28.80$ & $-17: 52: 00.8$ & 270 & TE-VF & 012367 & David Helfand & acisD2006-08-01t_gainN0006 \\
\hline W33 & 16674 & 2015-07-27T21:36 & 38188 & I3 & $18: 14: 13.49$ & $-17: 55: 41.9$ & 262 & TE-VF & 01237 & Leisa Townsley & acisD2015-05-01t_gainN0006 \\
\hline Cl $1813-178$ & 17695 & 2016-05-29T22:12 & 12886 & I3 & $18: 13: 24.29$ & $-17: 53: 30.8$ & 103 & TE-VF & 01237 & Gordon Garmire & acisD2016-02-01t_gain_biN0002 \\
\hline Cl $1813-178$ & 17440 & 2016-06-05T21:05 & 16725 & I3 & $18: 13: 24.29$ & $-17: 53: 30.8$ & 103 & TE-VF & 012367 & Gordon Garmire & acisD2016-02-01t_gain_biN0002 \\
\hline NGC 7538 & & & & & & & & & & & \\
\hline NGC7538 & 5373 & 2005-03-25T22:55 & 28785 & I3 & $23: 13: 45.49$ & $+61: 28: 16.6$ & 19 & TE-VF & 012367 & Gordon Garmire & acisD2005-02-01t_gainN0006 \\
\hline G333 & & & & & & & & & & & \\
\hline IGR J16195-4945 & 5471 & 2005-04-29T17:25 & 4750 & I3 & $16: 19: 30.00$ & $-49: 45: 00.0$ & 42 & TE-F & 012367 & John Tomsick & acisD2005-02-01t_gainN0006 \\
\hline AX J162246-4946 & 8161 & 2007-06-13T15:01 & 2900 & S3 & $16: 22: 46.99$ & $-49: 46: 55.1$ & 329 & $\mathrm{TE}-\mathrm{F}$ & 235678 & Stephen Murray & acisD2007-05-01t_gainN0006 \\
\hline AX J162208-5005 & 8141 & 2007-06-21T02:36 & 1529 & S3 & $16: 22: 08.39$ & $-50: 05: 41.9$ & 316 & TE-F & 235678 & Stephen Murray & acisD2007-05-01t_gainN0006 \\
\hline AX J162011-5002 & 9602 & 2008-05-28T19:52 & 1531 & S3 & $16: 20: 11.50$ & $-50: 02: 09.6$ & 358 & TE-F & 235678 & Stephen Murray & acisD2008-05-01t_gainN0006 \\
\hline AX J162046-4942 & 10507 & 2009-01-26T20:06 & 3333 & S3 & $16: 20: 46.60$ & $-49: 42: 46.8$ & 97 & TE-F & 235678 & Bryan Gaensler & acisD2008-11-01t_gainN0006 \\
\hline G333.6-0.2 & 9911 & 2009-06-14T12:19 & 60096 & I3 & $16: 22: 09.19$ & $-50: 06: 03.4$ & 327 & TE-VF & 012367 & Leisa Townsley & acisD2009-05-01t_gainN0006 \\
\hline PSR J1622-49 & 10929 & 2009-07-10Т07:21 & 19879 & I3 & $16: 22: 52.90$ & $-49: 49: 35.1$ & 292 & TE-F & 012367 & Nanda Rea & acisD2009-05-01t_gainN0006 \\
\hline G333.3-0.4 & 15617 & 2013-02-26Т01:12 & 26131 & I3 & $16: 21: 32.59$ & $-50: 24: 20.3$ & 79 & TE-VF & 012367 & Leisa Townsley & acisD2013-02-01t_gainN0006 \\
\hline G333.3-0.4 & 14532 & 2013-02-28T16:50 & 28204 & I3 & $16: 21: 32.59$ & $-50: 24: 20.3$ & 79 & TE-VF & 012367 & Leisa Townsley & acisD2013-02-01t_gainN0006 \\
\hline G333.1-0.4 & 14531 & 2013-06-25Т01:41 & 60271 & I3 & $16: 20: 39.49$ & $-50: 39: 58.6$ & 310 & TE-VF & 01237 & Leisa Townsley & acisD2013-05-01t_gainN0006 \\
\hline RCW 106 & 15393 & 2013-07-03Т00:22 & 59149 & I3 & $16: 20: 02.80$ & $-50: 58: 18.6$ & 294 & TE-VF & 01237 & Gordon Garmire & acisD2013-05-01t_gainN0006 \\
\hline AFGL 2591 & & & & & & & & & & & \\
\hline AFGL 2591 & 6442 & 2006-02-08Т01:26 & 29791 & S3 & $20: 29: 24.89$ & $+40: 11: 21.0$ & 16 & TE-VF & 235678 & Arnold Benz & acisD2006-02-01t_gainN0006 \\
\hline $\mathrm{G} 34.4+0.23$ & & & & & & & & & & & \\
\hline IRDC G34.4+0.23 & 14541 & 2013-06-17T12:03 & 28125 & I3 & $18: 53: 18.49$ & $+01: 24: 47.9$ & 146 & TE-VF & 01236 & Jonathan Tan & acisD2013-05-01t_gainN0006 \\
\hline IRDC G34.4+0.23 & 15664 & 2013-08-12T17:44 & 34526 & I3 & $18: 53: 18.49$ & $+01: 24: 47.9$ & 239 & TE-VF & 012367 & Jonathan Tan & acisD2013-08-01t_gainN0006 \\
\hline
\end{tabular}




\subsubsection{Point Source Detection Strategy}

As in previous studies, our point source detection workflow first identifies a liberal set of candidate sources, derived mostly from maximum likelihood image reconstruction, then iteratively prunes candidates found to be insignificant after extraction and careful local background estimation. Extraction apertures are normally sized to contain $90 \%$ of the PSF (at $1.5 \mathrm{keV}$ ), but are reduced when necessary to minimize overlap among crowded sources. (Aperture correction is discussed in Section 3.1.) Iteration is necessary because backgrounds and extraction apertures depend upon neighboring sources, so as insignificant source candidates are pruned from the catalog, remaining source candidates must be re-extracted and re-evaluated for validity.

The goals of completeness and validity of a source list are always in conflict. Our point source detection procedure is designed to be aggressive, emphasizing sensitivity and accepting a reasonable occurrence of possibly-spurious detections to achieve that sensitivity. In the CCCP study, Broos et al. (2011, Figure 9) showed that when deep near-IR catalogs are available, the fraction of X-ray detections without apparent near-IR counterparts rises only slowly as detection significance falls; this is indirect evidence that our procedures do not lead to a large number of false sources. Broos et al. (2011, Section 6.2) discuss the impracticality of quantifying the false detection rate in our X-ray catalog, and point out that such an estimate would be nearly useless because most science analyses select subsets of the X-ray catalog (e.g., sources with IR photometry available, or sources classified as young stars).

Detection of source candidates has changed in various ways since MOXC1. Since different ACIS pointings now commonly overlap considerably in our wide-field mosaics, pointing-based tiling has been eliminated. Now the whole field is tiled, tile sections are extracted from every ObsID, and only tile extractions with similar off-axis angles are merged, to avoid combining data with very different angular resolutions. These merged tile images, along with tile images from each constituent ObsID, are independently searched for sources.

We now use a more complex method to estimate the background in a reconstructed image, separately modeling particle and X-ray backgrounds. This accounts for the energy-dependent and time-dependent distribution of the X-ray background across the detector, caused by the spatially non-uniform and time-dependent contamination on the ACIS Optical Blocking Filter ${ }^{9}$.

Our procedure for searching reconstructed tile images for source candidates now involves several steps, in order to improve our ability to detect faint sources. After source candidates are identified in each reconstructed tile image, those candidates are removed, the resulting image is smoothed, and the smoothed image is searched again for fainter sources. At the end of the source-finding process, the resultant candidate source lists are combined (and duplicates removed); the final candidate source list for the entire target is then assessed for validity.

\subsubsection{Astrometric Alignment}

Since tremendous resources have been invested to achieve Chandra's superb angular resolution, we make considerable efforts to preserve that angular resolution by carefully aligning overlapping ACIS observations and assigning absolute astrometry by using the Two Micron All Sky Survey (2MASS, Skrutskie et al. 2006) catalog as the astrometric reference (this near-IR catalog works best for our

\footnotetext{
${ }^{9}$ http://cxc.harvard.edu/ciao/why/acisqecontamN0010.html
} 
obscured targets). This alignment work is iterative. The first round of alignment is performed by finding bright X-ray sources in each ObsID, then matching those independent single-ObsID catalogs of bright X-ray sources to each other and to the 2MASS catalog. (This matching scheme, considering relative astrometry shifts between ACIS ObsIDs as well as absolute astrometry shifts to align to 2MASS, is very helpful for aligning ACIS observations with a wide range of integration times; short ObsIDs are best aligned to longer ObsIDs, then the longer ObsIDs provide the absolute alignment to 2MASS.) A weighted analysis of the resulting offsets between pairs of matched sources produces recommended shifts for each ObsID; no rotational correction is attempted. Those shifts are applied, then the process is repeated until no further shifts are recommended. The final cumulative shifts are applied to the aspect calibration files for each ObsID and the event lists are reprojected onto the sky. Those single-ObsID catalogs of bright sources, which now have obsolete coordinates, are not considered further.

A fresh list of candidate sources (Section 2.2.1) is obtained from these aligned data, and the iterative process of extracting and validating those candidates begins. During this process we assess the alignment of ObsIDs at least twice more. As above, shift recommendations for each ObsID are obtained by combining error-weighted estimates of all pairs of inter-ObsID offsets and error-weighted estimates of single-ObsID offsets with respect to 2MASS; no rotational correction is attempted. We believe this second round of alignment work is worthwhile for two reasons. First, estimates of inter-ObsID shifts made at this point should be more accurate, because a single target-wide catalog is extracted from every ObsID - no matching is required. Second, the (single target-wide) catalog of source candidates available at this point, built after our first alignment of the data (described above), is expected to be a better representation of the sky (e.g., crowded sources are more likely to be resolved).

\subsubsection{Validation of Source Candidates}

For sources with multiple observations, Broos et al. (2010, Section 6.2) point out that a subset of the observations may produce higher quality measurements of source properties than the full set of observations would. This is often true when the observations have very different off-axis angles, and thus very different angular resolutions and degrees of crowding. As in previous studies, MOXC2 builds spectra and photometry from a set of observations that favors the photometric signal-to-noise ratio ${ }^{10}$ and estimates the source position from a set of observations that favors small position uncertainty.

In previous studies, source validation followed the same strategy; the validity of a source (in each energy band) was calculated from the set of observations in which the source was most valid. Broos et al. (2010, Section 6.2) point out that this selection process increases sensitivity to variable sources at the cost of an increased false detection rate arising from the additional number of data sets that are searched.

Recently, while reducing a Chandra target with a large number of overlapping ObsIDs, we found this source validation strategy to be inadequate, since the number of possible ways to combine $N$ ObsIDs grows rapidly with $N$. Thus, in MOXC2 we have adopted a different strategy to balance false detection rate and sensitivity to variable sources. Source validity is now evaluated on a predefined, small set of ObsID combinations. Those combinations do not depend on characteristics of

\footnotetext{
10 The "MERGE_FOR_PHOTOMETRY" option for combining ObsIDs in $A E$ tries to balance the goals of maximum
} signal-to-noise ratio and zero photometry bias. 
the data; they consist simply of (1) each ObsID by itself and (2) ObsIDs with similar off-axis angles. Empirically-determined off-axis angle ranges are $0^{\prime}-3^{\prime}, 2^{\prime}-6^{\prime}, 5^{\prime}-9^{\prime}, 8^{\prime}-14^{\prime}$, and $>13^{\prime}$. These ranges overlap to avoid missing sources at the boundaries.

As described in MOXC1, our procedures account for the Chandra PSF hook ${ }^{11}$, a structure that extends $\sim 0.8^{\prime \prime}$ from the main PSF peak and contains $\sim 5 \%$ of the flux. This artifact is significant for us, since our detection efforts emphasize faint sources and hooks around bright sources can be reconstructed as fainter neighboring candidate sources. We mark the hook location around bright sources, then visually examine those locations for source candidates that are consistent with the expected hook brightness. Such spurious "hook sources" are removed from the catalog.

\subsubsection{Diffuse Emission}

As always, our strategy for studying diffuse X-ray emission is to mask (remove) events that are likely to be associated with point sources, to subtract particle background, and to normalize by the exposure map (Townsley et al. 2003; Broos et al. 2010, Section 9). The resulting diffuse image represents observed surface brightness, which has units of photon $\mathrm{cm}^{-2} \mathrm{~s}^{-1} \operatorname{arcsec}^{-2}$. Masked data products (observed event list, particle background event list, and exposure maps) are built independently for each ObsID, and then combined into target-level images. A surface brightness image is computed from those observed, background, and exposure map images using an adaptive smoothing algorithm (Townsley et al. 2003; Broos et al. 2010, Section 9.1), in which the smoothing kernel is sized to achieve a target signal-to-noise ratio in the surface brightness.

The adaptively-smoothed X-ray flux images presented below (Section 4) cover the energy band 0.5-7.0 $\mathrm{keV}^{12}$ and are smoothed to a signal-to-noise ratio of 15 . The exposure map used in these images represents the instrument response at $1 \mathrm{keV}$.

\footnotetext{
11 http: //cxc.harvard.edu/ciao/caveats/psf_artifact.html

12 For diffuse images, the "full band" omits the energy range $7-8 \mathrm{keV}$ to avoid a bright emission line in the instrumental background.
} 


\section{MOXC2 DATA PRODUCTS}

\subsection{The MOXC2 Chandra Point Source Catalog}

The primary data product of MOXC2 is a catalog of properties for 18,396 point sources found in our 13 ACIS mosaics of MSFRs and their surrounding Galactic Plane environments. This catalog provides the same source information as that given in MOXC1 and is similar to catalogs from CCCP (Broos et al. 2011), MYStIX (Kuhn et al. 2013), and SFiNCs (Getman et al. 2017).

Table 3 defines the columns of the MOXC2 point source catalog. This catalog is available in FITS format from the electronic edition of this article and may be available in many other formats from VizieR (Ochsenbein et al. 2000). All photometric quantities in this table are apparent (not corrected for absorption). The suffixes "_t", "s", and "h" on names of photometric quantities designate the total $(0.5-8 \mathrm{keV})$, soft $(0.5-2 \mathrm{keV})$, and hard (2-8 keV) energy bands. Energy-dependent correction for finite extraction apertures is applied to the ancillary reference file (ARF) calibration products (see Broos et al. 2010, Section 5.3); the SrcCounts and NetCounts quantities characterize the extraction and are not aperture-corrected. The only calibrated quantities in the catalog are apparent photon flux in units of photon $\mathrm{cm}^{-2} \mathrm{~s}^{-1}$ (see Broos et al. 2010, Section 7.4), and an estimate for apparent energy flux in units of erg $\mathrm{cm}^{-2} \mathrm{~s}^{-1}$ (Getman et al. 2010). Table notes provide additional information regarding the definition of source properties.

We caution potential users of these results that we have not attempted to clean the point source catalog for sources that might be spurious reconstruction peaks, for example over-reconstructions of diffuse emission. This can be a problem for bright PWNe, SNRs, and dust-scattering halos around X-ray binaries. As we describe each target mosaic in Section 4 below, we will point out areas of particular concern for such spurious point sources.

Table 3. MOXC X-ray Sources and Properties

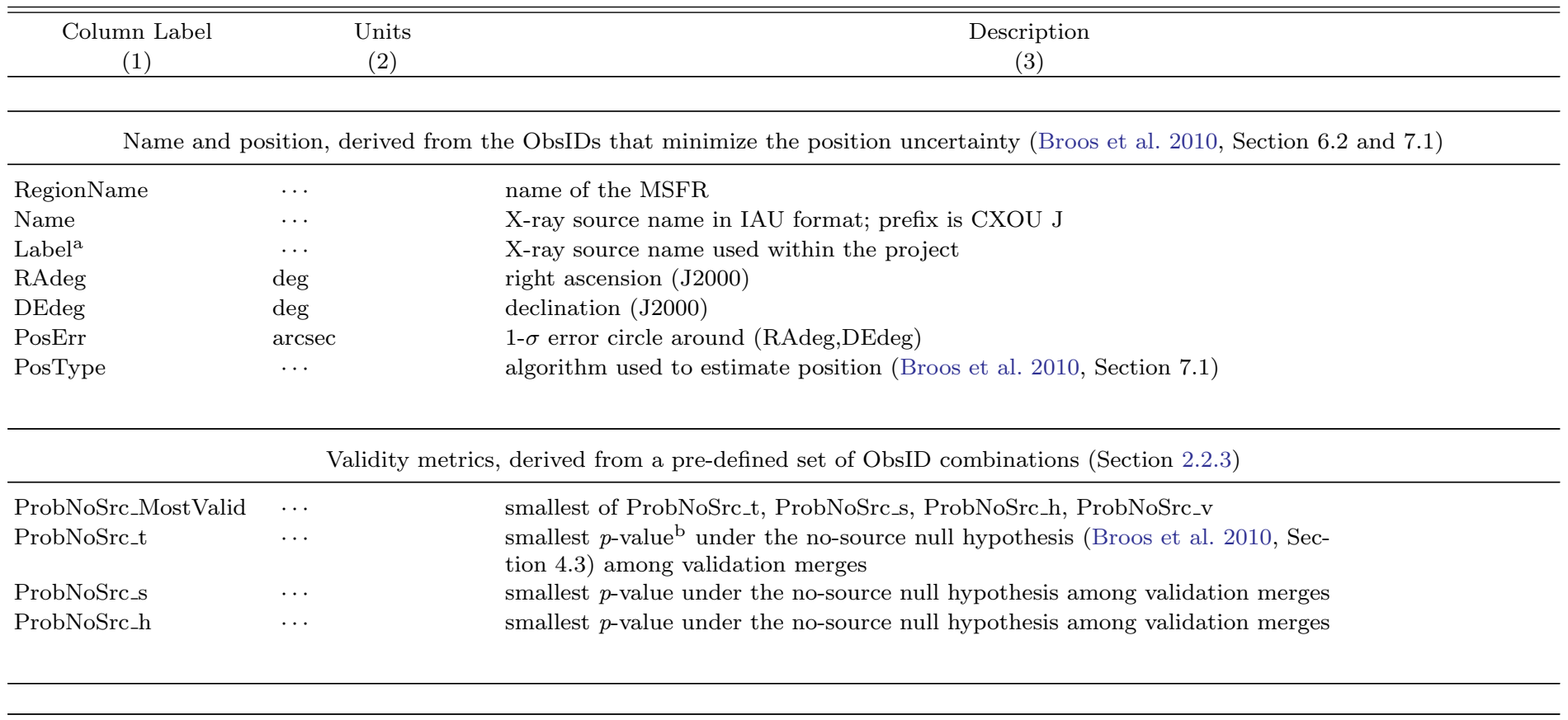


Table 3 (continued)

\begin{tabular}{|c|c|c|}
\hline $\begin{array}{c}\text { Column Label } \\
(1)\end{array}$ & $\begin{array}{c}\text { Units } \\
(2)\end{array}$ & $\begin{array}{c}\text { Description } \\
(3) \\
\end{array}$ \\
\hline & & Variability indices, derived from all ObsIDs \\
\hline ProbKS_single ${ }^{\mathrm{c}}$ & $\cdots$ & $\begin{array}{l}\text { smallest } p \text {-value under the null hypothesis (no variability within each single } \\
\text { ObsID) for the Kolmogorov-Smirnov test on the timestamps of each ObsID's } \\
\text { event list }\end{array}$ \\
\hline ProbKS_merge ${ }^{\mathrm{c}}$ & $\cdots$ & $\begin{array}{l}p \text {-value under the null hypothesis (no variability) for the Kolmogorov-Smirnov } \\
\text { test on the timestamps of the multi-ObsID event list }\end{array}$ \\
\hline ProbChisq_PhotonFlux & $\cdots$ & $\begin{array}{l}p \text {-value under the null hypothesis (no variability) for the } \chi^{2} \text { test on the single- } \\
\text { ObsID measurements of PhotonFlux_t }\end{array}$ \\
\hline \multicolumn{3}{|c|}{ Observation details and photometric quantities, derived from the set of ObsIDs that optimizes photometry (Broos et al. 2010, Section 6.2 and 7) } \\
\hline ExposureTimeNominal & $\mathrm{s}$ & total exposure time in merged ObsIDs \\
\hline ExposureFraction $^{\mathrm{d}}$ & $\cdots$ & fraction of ExposureTimeNominal that source was observed \\
\hline RateIn3x3Cell & count/frame & $0.5: 8 \mathrm{keV}$, in $3 \times 3 \mathrm{CCD}$ pixel cell \\
\hline NumObsIDs & $\ldots$ & total number of ObsIDs extracted \\
\hline NumMerged & $\cdots$ & number of ObsIDs merged to estimate photometry properties \\
\hline MergeBias & $\cdots$ & fraction of exposure discarded in merge \\
\hline Theta_Lo & $\operatorname{arcmin}$ & smallest off-axis angle for merged ObsIDs \\
\hline Theta & $\operatorname{arcmin}$ & average off-axis angle for merged ObsIDs \\
\hline Theta_Hi & $\operatorname{arcmin}$ & largest off-axis angle for merged ObsIDs \\
\hline PsfFraction & $\cdots$ & average PSF fraction (at $1.5 \mathrm{keV}$ ) for merged ObsIDs \\
\hline SrcArea & $(0.492 \operatorname{arcsec})^{2}$ & average aperture area for merged ObsIDs \\
\hline AfterglowFraction ${ }^{\mathrm{f}}$ & $\cdots$ & suspected afterglow fraction \\
\hline SrcCounts_t & count & observed counts in merged apertures \\
\hline SrcCounts_s & count & observed counts in merged apertures \\
\hline SrcCounts_h & count & observed counts in merged apertures \\
\hline BkgScaling & $\cdots$ & scaling of the background extraction (Broos et al. 2010, Section 5.4) \\
\hline BkgCounts_t & count & observed counts in merged background regions \\
\hline BkgCounts_s & count & observed counts in merged background regions \\
\hline BkgCounts_h & count & observed counts in merged background regions \\
\hline NetCounts_t & count & net counts in merged apertures \\
\hline NetCounts_s & count & net counts in merged apertures \\
\hline NetCounts_h & count & net counts in merged apertures \\
\hline NetCounts_Lo_t ${ }^{\mathrm{g}}$ & count & 1- $\sigma$ lower bound on NetCounts_t \\
\hline NetCounts_Hi_t & count & $1-\sigma$ upper bound on NetCounts_t \\
\hline NetCounts_Lo_s & count & 1- $\sigma$ lower bound on NetCounts_s \\
\hline NetCounts_Hi_s & count & 1- $\sigma$ upper bound on NetCounts_s \\
\hline NetCounts_Lo_h & count & $1-\sigma$ lower bound on NetCounts_h \\
\hline NetCounts_Hi_h & count & 1- $\sigma$ upper bound on NetCounts_h \\
\hline MeanEffectiveArea_t ${ }^{\mathrm{h}}$ & $\mathrm{cm}^{2}$ count photon ${ }^{-1}$ & mean $\mathrm{ARF}$ value \\
\hline MeanEffectiveArea_s & $\mathrm{cm}^{2}$ count photon ${ }^{-1}$ & mean $\mathrm{ARF}$ value \\
\hline MeanEffectiveArea_h & $\mathrm{cm}^{2}$ count photon ${ }^{-1}$ & mean $\mathrm{ARF}$ value \\
\hline MedianEnergy_t $\mathrm{t}^{\mathrm{i}}$ & $\mathrm{keV}$ & median energy, observed spectrum \\
\hline MedianEnergy_s & $\mathrm{keV}$ & median energy, observed spectrum \\
\hline
\end{tabular}


Table 3 (continued)

\begin{tabular}{|c|c|c|}
\hline $\begin{array}{c}\text { Column Label } \\
(1) \\
\end{array}$ & $\begin{array}{c}\text { Units } \\
(2) \\
\end{array}$ & $\begin{array}{c}\text { Description } \\
(3) \\
\end{array}$ \\
\hline MedianEnergy_h & $\mathrm{keV}$ & median energy, observed spectrum \\
\hline PhotonFlux_t ${ }^{\mathrm{j}}$ & photon $\mathrm{cm}^{-2} \mathrm{~s}^{-1}$ & apparent photon flux \\
\hline PhotonFlux_s & photon $\mathrm{cm}^{-2} \mathrm{~s}^{-1}$ & apparent photon flux \\
\hline PhotonFlux_h & photon $\mathrm{cm}^{-2} \mathrm{~s}^{-1}$ & apparent photon flux \\
\hline EnergyFlux_t & erg $\mathrm{cm}^{-2} \mathrm{~s}^{-1}$ & $\max ($ EnergyFlux_s,0) $+\max ($ EnergyFlux_h,0 $)$ \\
\hline EnergyFlux_sk & erg $\mathrm{cm}^{-2} \mathrm{~s}^{-1}$ & apparent energy flux \\
\hline EnergyFlux_hk & erg $\mathrm{cm}^{-2} \mathrm{~s}^{-1}$ & apparent energy flux \\
\hline
\end{tabular}

Note - These X-ray columns are produced by the ACIS Extract (AE) software package (Broos et al. 2010, 2012). Similar column labels were previously published by the CCCP (Broos et al. 2011) and by MOXC1 (Townsley et al. 2014). The AE software and User's Guide are available at http://personal.psu.edu/psb6/TARA/ae_users_guide.html.

Note- The suffixes "_t", "_s", and "_h" on names of photometric quantities designate the total (0.5-8 keV), soft (0.5-2 keV), and hard $(2-8 \mathrm{keV})$ energy bands.

Note- Source name and position quantities (Name,RAdeg, DEdeg, PosErr,PosType) are computed using a subset of each source's extractions chosen to minimize the position uncertainty (Broos et al. 2010, Section 6.2 and 7.1). Source significance quantities (ProbNoSrc_MostValid, ProbNoSrc_t, ProbNoSrc_s, ProbNoSrc_h) are computed using a pre-defined set of ObsID combinations, which do not depend on the data observed (Section 2.2.3). Variability indices (ProbKS_single, ProbKS_merge, ProbChisq_PhotonFlux ) are computed using all ObsIDs. All other quantities are computed using a subset of ObsIDs chosen, independently for each source, to balance the conflicting goals of minimizing photometric uncertainty and of avoiding photometric bias (Broos et al. 2010, Section 6.2 and 7).

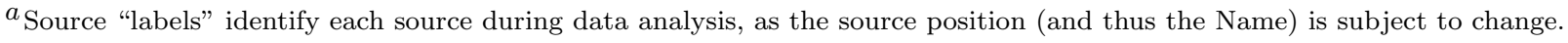

${ }^{b}$ In statistical hypothesis testing, the $p$-value is the probability of obtaining a test statistic at least as extreme as the one that was actually observed, when the null hypothesis is true. The $p$-value of the observed extraction under the no-source hypothesis is calculated by the method described by Weisskopf et al. (2007, Appendix A2), which is derived under the assumption that X-ray extractions follow Poisson distributions.

${ }^{c}$ See Broos et al. (2010, Section 7.6) for a description of the ProbKS_single and ProbKS_merge variability indexes, and caveats regarding possible spurious indications of variability using the ProbKS_merge index. The ProbChisq_PhotonFlux variability index is the $p$-value under the null hypothesis (no variability) for the standard $\chi^{2}$ test on the single-ObsID measurements of PhotonFlux_t.

$d$ Due to dithering over inactive portions of the focal plane, a Chandra source often is not observed during some fraction of the nominal exposure time. (See http://cxc.harvard.edu/ciao/why/dither.html.) The reported quantity is FRACEXPO, produced by the CIAO tool mkarf.

${ }^{e}$ ACIS suffers from a non-linearity at high count rates known as photon pile-up, described in Section 3.2 below. RateIn3x3Cell is an estimate of the observed count rate falling on an event detection cell of size $3 \times 3$ ACIS pixels, centered on the source position. When RateIn3x3Cell > 0.05 (count/frame), the reported source properties may be biased by pile-up effects. See Table 4 for a list of MOXC2 sources with significant pile-up. All source properties in this table are not corrected for pile-up effects.

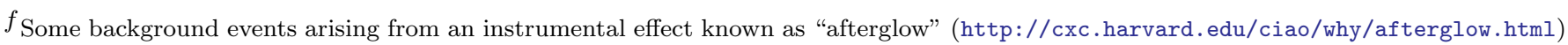
may contaminate source extractions, despite careful procedures to identify and remove them during data preparation (Broos et al. 2010, Section 3). After extraction, we attempt to identify afterglow events using the AE tool ae_afterglow_report, and report the fraction of extracted events attributed to afterglow; see the ACIS Extract manual (http://personal.psu.edu/psb6/TARA/ae_users_guide.html).

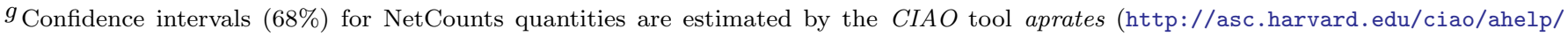
aprates.html).

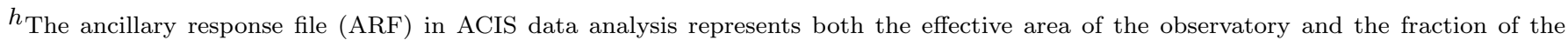
observation for which data were actually collected for the source (ExposureFraction).

${ }^{i}$ MedianEnergy is the median energy of extracted events, corrected for background (Broos et al. 2010, Section 7.3).

$j$ PhotonFlux $=($ NetCounts / MeanEffectiveArea / ExposureTimeNominal) (Broos et al. 2010, Section 7.4).

${ }^{k}$ EnergyFlux $=1.602 \times 10^{-9}(\mathrm{erg} / \mathrm{keV}) \times$ PhotonFlux $\times$ MedianEnergy (Getman et al. 2010, Section 2.2). 


\subsection{Piled Sources}

As described in detail in MOXC1, ACIS detections of bright X-ray sources can suffer from a nonlinearity known as photon pile-up ${ }^{13}$, where multiple X-ray photons are mistakenly detected as a single event because they fell close to each other on the CCD and arrived during the same readout frame. Pile-up causes photometry to be underestimated and the spectrum to be hardened. We check for pileup in every observation of a source by estimating the observed count rate in a $3 \times 3$ pixel detection cell centered on the source position. (The highest rate found in all observations of the source is reported in the column RateIn3x3Cell in Table 3.)

For each source extraction in which RateIn3x3Cell exceeded a threshold of 0.05 count/frame, we modeled pile-up using a Monte Carlo approach that reconstructs a pile-up-free spectrum from a piled ACIS observation (Broos et al. 2011). Table 4 lists those extractions; column (8) characterizes the inferred level of pile-up ${ }^{14}$. For those sources, several entries in Table 3 are expected to be biased by pile-up effects (in an energy-dependent way). Users are warned that photometric quantities for piledup sources should be used with caution; higher pile-up corrections and narrower bandpasses should evoke the most caution. Alternatively, we provide pileup-corrected spectra for all source/ObsID entries in Table 4, as described below. Fitting those spectra will result in more meaningful source properties than possible from pileup-distorted photometry.

\subsection{Archive of Reduced Data Products}

The Zenodo data repository ${ }^{15}$ archives many MOXC2 reduced data products ${ }^{16}$ (Townsley \& Broos 2017), including astrometrically aligned event lists and exposure maps, DS9 region files representing point source extraction apertures and PSF hooks, source photometry in 16 energy bands, reconstructed spectra for sources that suffer from photon pile-up (Section 3.2), lightcurve plots for the brighter sources, event lists and exposure maps with point sources masked, and smoothed images of diffuse emission (Section 4). For the piled sources in Table 4, we provide reconstructed spectra for each ObsID in which the source suffered from pile-up. The "README" file in the Zenodo archive describes the files there.

\subsection{MOXC2 Sources in Published Chandra Catalogs}

Many of the brighter X-ray point sources in MOXC2 also appear in the Chandra Source Catalog ${ }^{17}$ (Evans et al. 2010). Some may also have data available from the XMM-Newton mission's EPIC camera; catalogs of $X M M$ sources are available from the XMM-Newton Survey Science Centre ${ }^{18}$. In crowded regions such as MSFRs, care should be taken in matching MOXC2 sources to XMM sources due to the mismatch in spatial resolution between the Chandra and XMM telescopes.

Several short archival Chandra observations in and around MSFRs were obtained by the snapshot survey Chasing the Identification of ASCA Galactic Objects (ChIcAGO, Anderson et al. 2014). We use these datasets in the MOXC2 mosaics and the brighter X-ray sources in these fields are contained in the ChIcAGO catalog. Several examples are mentioned in the descriptions of individual MSFRs below.

\footnotetext{
13 http://cxc.harvard.edu/ciao/why/pileup_intro.html

14 We choose not to use the terms "pile-up fraction" or "pile-up percentage" because the ACIS community has several conflicting definitions for those terms; see Section 1.2 in The Chandra ABC Guide to Pileup (http://cxc. harvard.edu/ciao/download/doc/pileup_abc.pdf).

15 https://zenodo.org

16 https://doi.org/10.5281/zenodo.1067749

17 http://cxc.cfa.harvard.edu/csc/

18 http://xmmssc.irap.omp.eu
} 
Table 4. Sources Exhibiting Photon Pile-up

\begin{tabular}{|c|c|c|c|c|c|c|c|}
\hline MSFR & $\begin{array}{c}\text { Name } \\
(\mathrm{CXOU} J)\end{array}$ & Label & Identifier & ObsID & $\begin{array}{c}\theta \\
\left({ }^{\prime}\right)\end{array}$ & PsfFraction & Correction \\
\hline (1) & $(2)$ & $(3)$ & $(4)$ & $(5)$ & $(6)$ & $(7)$ & $(8)$ \\
\hline \multirow[t]{5}{*}{ NGC 6334} & 171701.53-362100.6 & GM24_c717 & VVV J171701.53-362100.67 & 18876 & 1.9 & 0.64 & 1.027 \\
\hline & 171946.16-360552.2 & c1934 & HD 319703A & 12382 & 3.1 & 0.89 & 1.015 \\
\hline & 171946.16-360552.2 & c1934 & HD 319703A & 13436 & 3.1 & 0.89 & 1.033 \\
\hline & 172001.73-355816.2 & c3105 & 2MASS J17200173-3558162 & 2573 & 1.9 & 0.90 & 1.024 \\
\hline & 172031.75-355111.4 & c5437 & 2MASS J17203178-3551111 & 8975 & 2.5 & 0.90 & 1.121 \\
\hline RCW 120 & 171405.73-381031.4 & c2911 & pulsar CXOU J171405.7-381031 & 6692 & 0.4 & 0.89 & 1.226 \\
\hline \multirow[t]{2}{*}{ W31N } & 180839.35-202439.9 & c633 & SGR 1806-20 & 1827 & 0.6 & 0.89 & 2.543 \\
\hline & 180839.35-202439.9 & c633 & SGR 1806-20 & 6224 & 9.8 & 0.50 & 1.056 \\
\hline IRAS $19410+2336$ & $194154.60+225112.3$ & $\mathrm{c} 19$ & ChI J194152+2251_2 $2^{\text {a }}$ & 8164 & 0.7 & 0.46 & $\ldots$ \\
\hline \multirow[t]{2}{*}{ W42; RSGC1 } & $183803.17-065533.7$ & c989 & PSR J1838-0655 & 16673 & 7.2 & 0.90 & 1.031 \\
\hline & 183803.17-065533.7 & c989 & PSR J1838-0655 & 6719 & 5.3 & 0.90 & 1.126 \\
\hline W33; Cl 1813-178 & 181335.16-174957.4 & $\mathrm{c} 2292$ & PSR J1813-1749 & 6685 & 2.8 & 0.90 & 1.066 \\
\hline \multirow[t]{4}{*}{ G333 } & $161932.20-494430.6$ & c439 & HMXB IGR J16195-4945 & 5471 & 0.4 & 0.91 & $\cdots$ \\
\hline & $162006.69-500158.8$ & c1594 & ChI J162011-5002_1 ${ }^{\mathrm{a}}$ & 9602 & 1.0 & 0.90 & 1.174 \\
\hline & $162048.81-494215.0$ & c3690 & ChI J162046-4942_1 ${ }^{\mathrm{a}}$ & 10507 & 0.6 & 0.89 & 1.137 \\
\hline & 162244.91-495052.8 & c8972 & magnetar PSR J1622-4950 & 8161 & 4.0 & 0.89 & 1.146 \\
\hline \multirow[t]{2}{*}{ Wd1 } & $164710.18-455216.8$ & c1081 & magnetar CXOU J164710.2-455216 & 5411 & 1.3 & 0.89 & 1.062 \\
\hline & $164710.18-455216.8$ & c1081 & magnetar CXOU J164710.2-455216 & 6283 & 1.8 & 0.89 & 1.055 \\
\hline
\end{tabular}

$a$ "ChI" sources are from the ChIcAGO project (Chasing the Identification of ASCA Galactic Objects, Anderson et al. 2014).

Note- Col. (1): Name of the MSFR.

Col. (2): X-ray source name in IAU format (Name in Table 3).

Col. (3): X-ray source name used within the project (Label in Table 3).

Col. (4): Source counterpart from VizieR or SIMBAD.

Col. (5): Chandra Observation Identification.

Col. (6): Off-axis angle (Theta in Table 3).

Col. (7): Fraction of the PSF (at $1.497 \mathrm{keV}$ ) enclosed within the extraction region (PsfFraction in Table 3). A reduced PSF fraction (significantly below $90 \%$ ) indicates that the source is in a crowded region.

Col. (8): Estimated ratio of pile-up-free to observed (piled) count rates in the $0.5-8 \mathrm{keV}$ energy band. This correction can be applied to the quantities SrcCounts_t, NetCounts_t, and PhotonFlux_t in Table 3. No value is reported when pile-up correction could not be performed. Note that the core of any source is more piled than the wings, so this correction factor depends on the aperture size (Column 7).

We have one target (RCW 120) in common with SFiNCs (Getman et al. 2017) and two targets (NGC 6334 and G333) for which a subset of MOXC2 ObsIDs were included in MOXC1; comparisons of these catalogs are mentioned briefly in the specific target descriptions of Section 4. A few other individual MOXC2 MSFRs have published Chandra point source catalogs. MOXC2 typically recovers all or most of these sources and goes on to find additional faint sources; again details are given in Section 4. 


\section{X-RAY CHARACTERIZATIONS OF MOXC2 TARGETS}

Following the format we established in MOXC1, we now provide short vignettes of each MSFR included in $\mathrm{MOXC2}$, to illustrate the distribution of X-ray point sources in the Chandra mosaics and to give a qualitative sense of the diffuse X-ray emission present in and around these fields. We do not attempt comprehensive reviews of the literature, which is often vast and multi-generational for these famous targets; rather we provide only the most cursory set of references, focusing on recent papers and X-ray studies. These references (and the papers they cite) provide a much better introduction to the MOXC2 MSFRs than space allows here. We also do not attempt extensive quantitative analysis either of the MSFR X-ray source populations or diffuse X-ray emission; such work is beyond the scope of MOXC2 and must await future efforts by ourselves and the wider star formation community.

For each target we show two basic figures (in celestial J2000 coordinates): the distribution of brighter ( $\geq 5$ net counts) X-ray sources on the exposure map of our ACIS mosaic (with ObsID numbers given in blue) and an adaptively-smoothed image of unresolved X-ray emission presented in the context of mid-IR data. In the first of these, X-ray sources are represented as colored dots, with the color indicating the median energy of the extracted X-rays. The legend shows the number of sources plotted in each median energy range. The hardest sources were plotted last, so some softer sources may be covered by the symbols for harder sources. Fainter sources are not included because their spatial distribution is strongly dependent on Chandra sensitivity (their numbers necessarily fall off sharply with off-axis angle). The number of faint sources can be calculated as the total number of detected sources (noted on the figure below the target name) minus the numbers given in the legend. Since we have made great efforts to detect the faintest X-ray sources possible, most targets have well over half of their ACIS detections absent from these plots. They do, however, serve to indicate important clumps and clusters of X-ray sources that trace the structure of the MSFRs they sample. Often those groupings of X-ray sources are shown in more detail in further images that show soft and hard X-ray events in the context of unresolved X-ray emission or Spitzer/IRAC $8 \mu \mathrm{m}$ structures.

The second basic figure shown for each target is a three-color image intended to place unresolved Xray emission in the context of the cold ISM traced by mid-IR images from Spitzer or WISE. In many instances, we include zoomed panels of this three-color image to highlight particularly interesting regions, with more informative image scaling tailored specifically for the diffuse X-ray emission in those regions. For diffuse emission, we consider "full-band" to be $0.5-7 \mathrm{keV}$, not the $0.5-8 \mathrm{keV}$ used for point source photometry, because the ACIS particle background ${ }^{19}$ rises sharply above $7 \mathrm{keV}$.

Since MSFRs are full of stars and all of our Chandra observations are too shallow to trace the complete initial mass function of a MSFR, we expect some of the unresolved X-ray emission (especially in cluster centers) to come from pre-MS stars. It is clear from these images, however, that we are teasing out faint, truly diffuse X-ray emission in these complexes, as demonstrated by the dramatic anticoincidence of the unresolved X-ray emission with extended IR structures. Thus, for convenience, we will refer to unresolved X-ray emission as "diffuse," keeping in mind that this is simply shorthand for "a mix of unresolved point source emission and truly diffuse X-ray structures."

As mentioned above, we additionally show the ACIS binned event data in two false-color images for selected regions of each target. These are often cluster centers or other interesting clumps of X-ray sources. For all such images, ACIS soft $(0.5-2 \mathrm{keV})$ events are shown in red, hard $(2-7 \mathrm{keV})$ events

19 http://cxc.cfa.harvard.edu/contrib/maxim/stowed/ 
in green; the binsize is one "sky pixel" $\left(0.5^{\prime \prime}\right.$; since ACIS X-ray events have real-valued positions, an image can be made with whatever binsize is helpful to the eye). In the superposition of these two event images, pixels that contain both soft and hard events appear yellow. Point source extraction regions are outlined with blue polygons. Those polygons may come from different ObsIDs, so they do not all have the same default size and orientation. Superposed on the event images is a blue image; this is either the ACIS full-band diffuse emission or Spitzer/IRAC $8 \mu \mathrm{m}$ emission.

Throughout this section, we occasionally note X-ray spectral fit parameters for massive stars or other bright X-ray sources of particular significance in MSFRs. Massive stars generate X-rays via a variety of emission mechanisms (e.g., Güdel \& Nazé 2009). Individual massive stars emit soft X-rays $(<1 \mathrm{keV})$ from shocks caused by velocity differences in their line-driven winds (see CCCP X-ray spectral fit examples in Gagné et al. 2011; Nazé et al. 2011). Magnetic massive stars can generate harder X-rays (up to a few keV) when their winds are channeled along magnetic field lines and collide (Babel \& Montmerle 1997). In massive binaries, hard X-rays (several keV) can be generated when the powerful winds from the two massive stars collide; these "colliding-wind binaries" (CWBs) can be bright X-ray sources and have been studied extensively with XMM-Newton and Chandra (e.g., Rauw \& Nazé 2016).

These X-ray spectral fits were performed with XSPEC (Arnaud 1996) usually employing the absorption model TBabs with solar abundances and the thermal plasma emission model apec; the model form used is TBabs*apec unless otherwise noted. Other bright X-ray sources are often collapsed objects with synchrotron X-ray emission; their X-ray spectra are fitted with absorbed power law models. The X-ray luminosities $\left(L_{X}\right)$ are intrinsic (absorption-corrected) and calculated for full-band (now $0.5-8 \mathrm{keV}$, the typical band used for ACIS point source fits in the literature). We intentionally do not give errors on fit parameters to emphasize that these are rough, preliminary characterizations of source spectra.

Since soft X-rays are readily absorbed by intervening material and such absorption may be strong (and spatially complex) in MSFRs, our estimates of X-ray luminosities are often lower limits. Especially in massive stars, soft X-ray plasma components are likely to be present but may be completely absorbed by heavy obscuration, thus they remain uncharacterized by our ACIS spectra. The same holds true for diffuse X-ray emission; its spatial complexity is probably due to a combination of absorption by intervening material and displacement of hot plasmas by colder ISM structures.

As described above, our analysis machinery uses image reconstruction to find candidate X-ray point sources. Regions of bright diffuse emission (e.g., PWNe or SNRs) can be erroneously overreconstructed in this process, leading to spurious point sources in our catalogs. We have chosen to leave such suspicious point sources in place, as long as they satisfy all point source validity criteria, because PWNe or SNRs can certainly have real X-ray point sources superposed on them. We caution users of our catalog to be aware of this decision and to employ more sophisticated means (such as searching for multiwavelength counterparts) to assess the validity of our sources that lie in close proximity to pulsars or in other regions of highly-structured, bright diffuse X-ray emission. 


\subsection{NGC 6334}

Using the example of the massive filamentary infrared dark cloud (IRDC) known as "Nessie," Jackson et al. (2010) suggested that multiple star formation sites can form at regular intervals along a cylindrical IRDC via the "sausage instability." This instability was originally described by Chandrasekhar \& Fermi (1953) and is essentially the analog of Jeans collapse in a self-gravitating sphere, applied now to a self-gravitating fluid cylinder. The dominant path for the intense star formation that results in MSFRs is now recognized to be such massive molecular filaments (Goodman et al. 2014), structures up to $100 \mathrm{pc}$ long formed by supersonic flows that compress the gas of the ISM into a web of dense, cylindrical structures criss-crossing GMCs (André et al. 2014). These pressurized "ridges" are crucibles for creating MSFRs; material flows down the filaments, feeding hot cores and clumps that eventually collapse to become massive stars and star clusters (Tackenberg et al. 2014).

Our closest example is the G352 GMC, a 90-pc-long massive filament made famous by Herschel (Russeil et al. 2013). G352 hosts the two multi-MSFR complexes NGC 6357 and NGC 6334 (Persi \& Tapia 2008); such complexes are known as "clusters of clusters" (e.g., Bastian et al. 2007; Elmegreen 2008). Each of these sports a string of MSFRs with multiple bubbles and degree-sized "bowls" filled with hot plasma (MOXC1); they are situated on opposite sides of G352's main filament.

NGC 6334 is the best nearby example we have of the ongoing transfiguration of such a massive molecular filament into stars; it is thought to contain 175 O-B3 stars (Russeil et al. 2012) distributed among several MSFRs. It hosts a large number of Hit regions of various sizes and evolutionary states, spread across $>2^{\circ}$ of the Galactic Plane (Russeil et al. 2016). Many of its most massive clusters line up along a dense molecular ridge with a complicated filament morphology and many subfilaments. In some of these subfilaments, densities are high enough that gravity should dominate the energetics and they should be collapsing to form stars (Russeil et al. 2013). This is substantiated by a recent IR study (Willis et al. 2013) that identifies 2283 Class I and II young stellar objects in NGC 6334; these sources tend to cluster along the subfilaments and extend many parsecs beyond the main ridge. As shown in MOXC1 and earlier studies, the three ACIS pointings along the main ridge capture several collapsing subfilaments and add a large number of Class III (diskless) pre-MS stars to the census of young stars populating those regions (Broos et al. 2013; Feigelson et al. 2009; Ezoe et al. 2006).

Recent maser parallax measurements by the VLBA BeSSeL Survey (Wu et al. 2014) and VERA (Chibueze et al. 2014) both yield a distance of $\sim 1.3 \mathrm{kpc}$ for NGC 6334, which we adopt for MOXC2. Readers should note, however, that some recent papers (e.g., Russeil et al. 2016; Tigé et al. 2017) use a distance of $\sim 1.75 \mathrm{kpc}$.

NGC 6334 serves as a transition target for us, illustrating the changes in our ACIS data analysis code and procedures between the MOXC1 analysis (circa 2013) and the current analysis for MOXC2. The same three ObsIDs analyzed for MOXC1 are re-analyzed here, augmented by several surrounding archival ACIS-I and ACIS-S pointings and our recent Chandra Cycle 17 General Observer (GO) data on the western side of NGC 6334's main filament, including the MSFR GM 24 (GM 1-24 in SIMBAD) (Tapia \& Persi 2008; Tapia et al. 2009).

Our final $1.4^{\circ}$-wide ACIS mosaic is shown in Figure 1. Chandra typically characterizes diskless young stellar populations, both older pre-MS stars distributed across MSFRs and younger sources still embedded in their natal clouds. That generalization appears to hold true for NGC 6334; widelydistributed X-ray sources are seen across the entire ACIS mosaic, extending far from the main ridge of MSFRs typically studied in NGC 6334. 


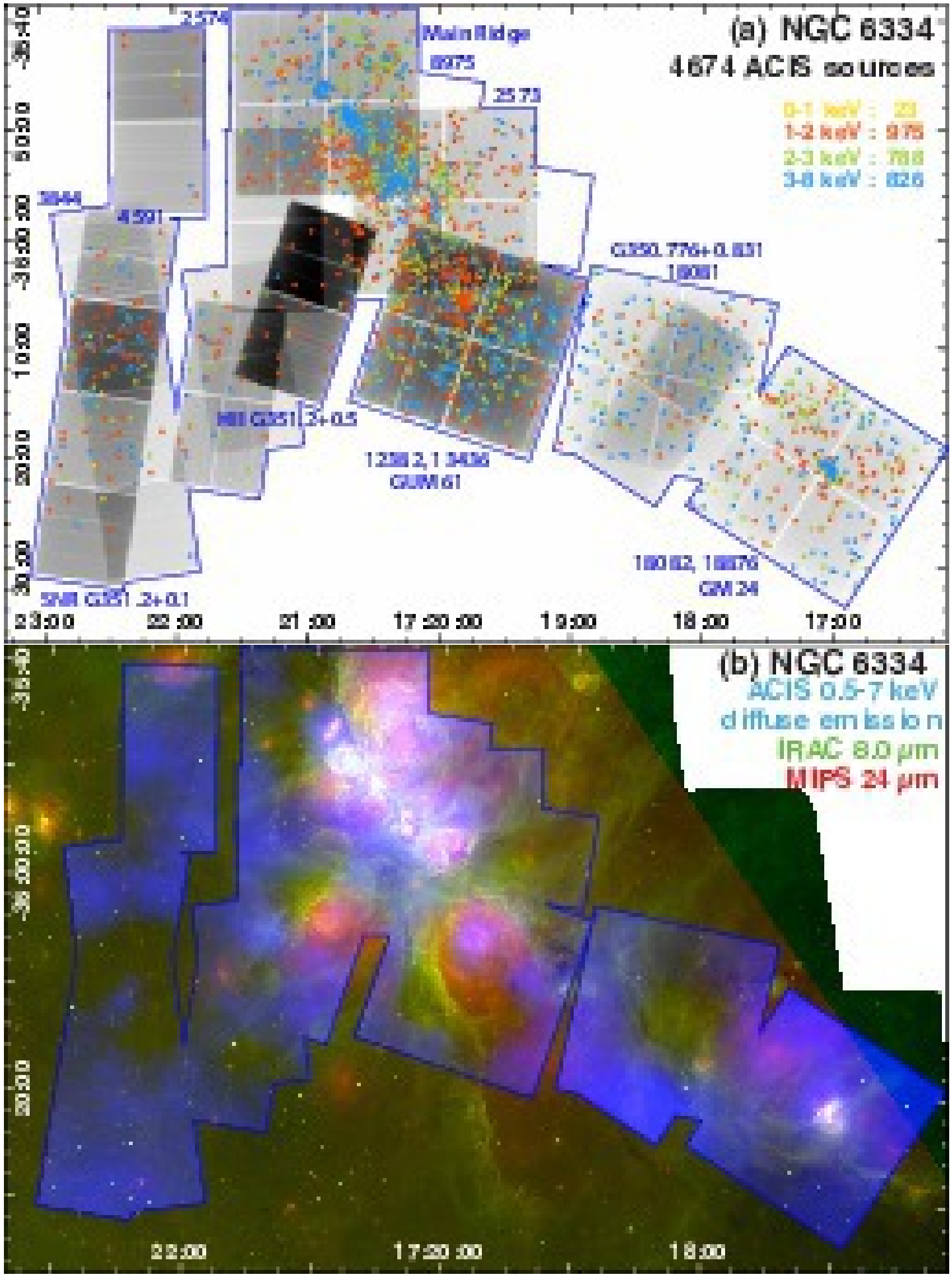

Figure 1. NGC 6334. (a) ACIS exposure map with brighter ( $\geq 5$ net counts) ACIS point sources overlaid; colors denote median energy for each source. ObsID numbers and regions named in the text are shown in blue. (b) ACIS diffuse emission in the Spitzer context. 
The original ACIS pointings on the main ridge have been described at length (Feigelson et al. 2009; Ezoe et al. 2006) and were presented in MOXC1, so we do not discuss them further here, except to note that they are pervaded by diffuse X-ray emission (Figure 2). Comparing the MOXC1 and MOXC2 catalogs in this region (where the two epochs of analysis used the same data), we find that both recover the same brighter sources ( $\geq 12$ net counts) at all off-axis angles. For faint sources, the catalogs differ in several ways. Sometimes one analysis split a group of counts into a close pair of sources while the other found a single source. Sometimes faint sources were deemed valid in one analysis but not the other; this is especially true at large $\left(>7^{\prime}\right)$ off-axis angles. These results are expected, given the strong interdependencies between faint candidate sources, the iterative nature of source validation, and the many decisions made, both algorithmic and by the analyst during visual reviews. Close examination of source positions shows that the inter-ObsID alignment is improved in MOXC2. Given this and the algorithmic improvements made over the years between MOXC1 and MOXC2, we recommend the use of the MOXC2 source list.

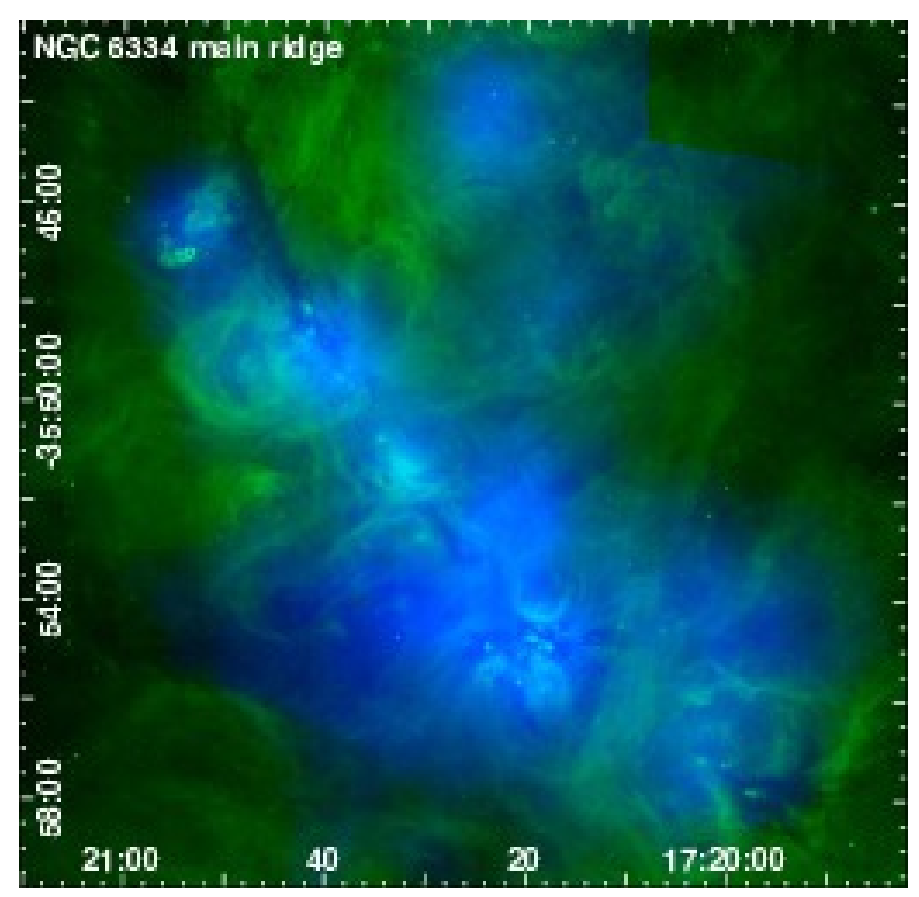

Figure 2. NGC 6334's main ridge of MSFRs. Zoomed version of Figure 1(b) for the central clusters on the main star-forming ridge, now with the MIPS data omitted because they are largely saturated. This figure is included to emphasize the extensive diffuse X-ray emission associated with this famous star-forming ridge.

\subsubsection{GUM 61}

Continuing down the G352 molecular filament southwest of the main ridge in NGC 6334, we find deep archival ACIS data on the large Hir region GUM 61 (Russeil et al. 2016) (Figure 3). This hosts a populous diffuse cluster, as shown by the large number of X-ray sources seen across the entire ACIS-I field in this pointing (Figure 1(a)).

At the center of the cluster (Figure 3(b)), the piled ACIS source c1934 (CXOU J171946.16-360552.2) matches HDE 319703A, a spectroscopic binary of type O7V((f)) + O9.5V (Maíz Apellániz et al. 2016). A spectral fit to the pileup-corrected spectrum of c1934 (from ObsID 13436) requires two thermal 

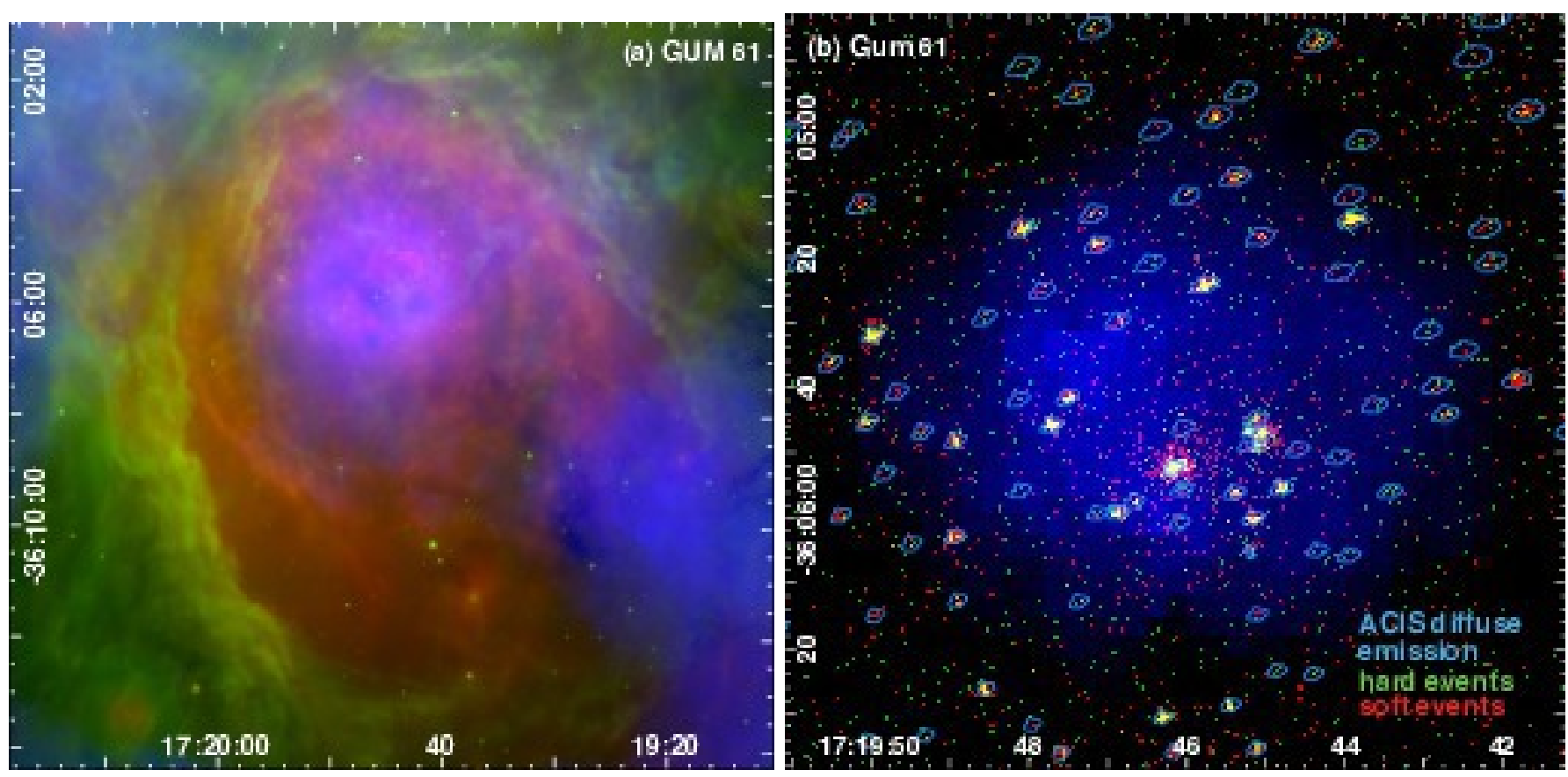

Figure 3. GUM 61. (a) Zoomed version of Figure 1(b) for the large Hir region GUM 61. (b) ACIS event data and diffuse emission for the center of GUM 61.

plasma components, TBabs(apec1 + apec2), for an acceptable fit. This yields $N_{H}=1.1 \times 10^{22} \mathrm{~cm}^{-2}$, $k T 1=0.7 \mathrm{keV}, k T 2=1.9 \mathrm{keV}$, and $L_{X}=1.6 \times 10^{32} \mathrm{erg} \mathrm{s}^{-1}$. The hard thermal plasma component dominates the spectrum. In ObsID 12382, obtained four days after ObsID 13436, c1934 shows slightly different spectral parameters $\left(N_{H}=1.4 \times 10^{22} \mathrm{~cm}^{-2}, k T 1=0.6 \mathrm{keV}, k T 2=2.5 \mathrm{keV}\right)$ and is brighter, with $L_{X}=2.0 \times 10^{32} \mathrm{erg} \mathrm{s}^{-1}$. The softer plasma component dominates the spectrum below $2 \mathrm{keV}$. A hard, variable X-ray spectrum and changing X-ray luminosity often indicate wind interactions in binary systems; this may be yet another CWB discovered by Chandra.

Nearby is HD 319703B, a visual binary of type O6V((f))z (Sana et al. 2014), matching ACIS source c1800 (CXOU J171945.05-360547.0). It has 432 net counts and a median energy of $1.3 \mathrm{keV}$. The spectral fit yields $N_{H}=1.4 \times 10^{22} \mathrm{~cm}^{-2}, k T=0.5 \mathrm{keV}$, and $L_{X}=9 \times 10^{31} \mathrm{erg} \mathrm{s}^{-1}$. Source c1800 has four close neighbors in the ACIS data. We also detect HD 319703D, spectral type O9.5: Vn (Sana et al. 2014), as ACIS source c2162 (CXOU J171948.94-360602.8), with 98 net counts and a median energy of $1.4 \mathrm{keV}$. For the spectral fit, we find $N_{H}=1.9 \times 10^{22} \mathrm{~cm}^{-2}, k T=0.6 \mathrm{keV}$, and $L_{X}=3 \times 10^{31} \mathrm{erg} \mathrm{s}^{-1}$.

There is prominent diffuse X-ray emission near these massive stars and at the southwest (open) edge of the IR bubble (Figure 3(a)), where Russeil et al. (2016) note outflow in the kinematics of this region. This is probably a good example of diffuse X-ray emission tracing hot gas from massive star feedback in an His region, flowing out of that region to enrich and heat the surrounding ISM. It might be compared to M17, a spectacular example of this phenomenon (Townsley et al. 2003, MOXC1).

\subsubsection{Southwest Pointings}

Extending the ACIS mosaic southwest along NGC 6334's natal molecular filament past GUM 61, our ACIS pointing named G350.776+0.831 (ObsID 18081) captures a complex network of subfilaments (Figure 4(a)) and known clumps of pre-MS stars (Russeil et al. 2013; Willis et al. 2013). It is centered 
on the base of a large "bowl" of bright $8 \mu \mathrm{m}$ emission seen opening to the northwest in Figure 1(b); the main molecular filament forming NGC 6334's backbone makes up the southern edge of this 30'diameter structure. This pointing shows substantial shadowing or displacement of hot plasma in a large oval region at the base of the bowl, but other parts of the bowl contain diffuse X-ray emission, as does the large elongated cavity on the southern side of the main filament, opening from Gum 61 to the southwest.

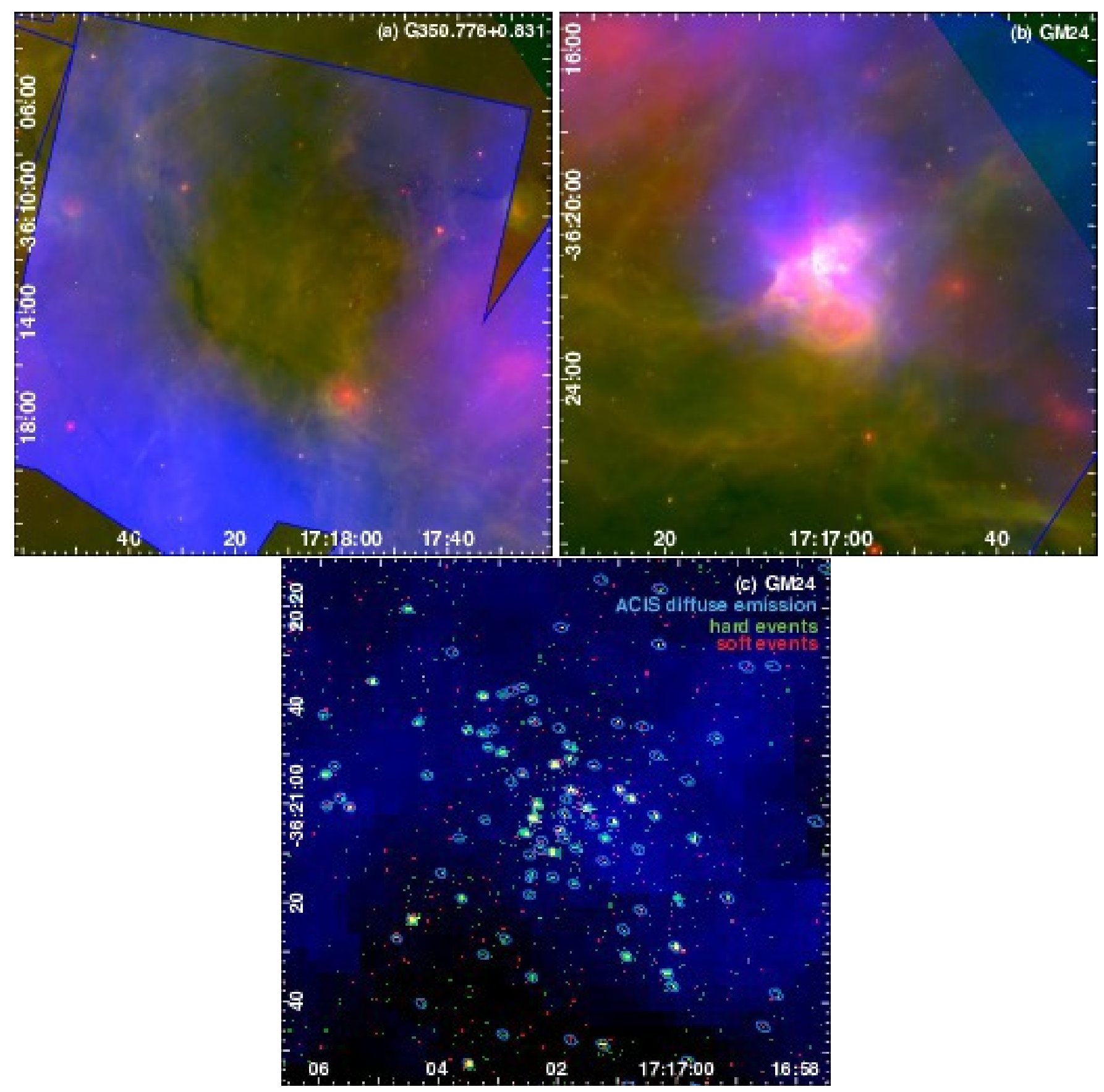

Figure 4. NGC 6334 southwest, along the giant filament. (a) Zoomed version of Figure 1(b) for the G350.776+0.831 region. (b) Zoomed version of Figure 1(b) for the isolated, very young MSFR GM 24. (c) ACIS event data and diffuse emission for the embedded cluster in GM 24. 
The ACIS mosaic continues down the main filament to the isolated, embedded IR cluster GM 24 (Figures 4(b) and (c)). This very young, midsized cluster $\left(\sim 250 \mathrm{M}_{\odot}\right)$ (Tapia et al. 2009$)$ is ionized by an O8 star (Bik et al. 2006); it sits at the southern edge of the Spitzer bowl, separated from the cluster-of-clusters complex by over 40'. GM 24 could have formed as a result of the bowl's dynamical influence on NGC 6334's main molecular filament. GM 24 appears to support a strong bipolar outflow that is carving cavities in the surrounding GMC (Tapia et al. 2009). ACIS finds a strong concentration of X-ray point sources coincident with GM 24, plus a large distributed population. Hot plasma clearly fills the western bowl above the main filament; this pointing also captures the edge of the southwestern elongated cavity and again finds diffuse X-ray emission there.

The piled ACIS source GM24_c717 (CXOU J171701.53-362100.6), near the center of GM 24, has a reduced extraction aperture due to a close, faint neighbor (c715) to the northwest. The position for the $\mathrm{O} 8$ star from Bik et al. (2006) is $3^{\prime \prime}$ north of GM24_c717, so it is not clear that this X-ray source is the counterpart of the $\mathrm{O} 8$ star. There is no X-ray source close to the O8 star's position.

We fit GM24_c717's pileup-corrected spectrum (from ObsID 18876) with the same simple spectral model used for other massive stars. Unlike the GUM 61 sources described above, GM24_c717 has a very hard spectrum, with $N_{H}=4 \times 10^{22} \mathrm{~cm}^{-2}, k T=9 \mathrm{keV}$, and $L_{X}=2 \times 10^{32} \mathrm{erg} \mathrm{s}^{-1}$. Any additional soft thermal plasma component could easily be absorbed away by this high column, so the luminosity we report is a lower limit. In the second GM 24 dataset taken three days later (ObsID 18082), the X-ray luminosity of GM24_c717 is lower by a factor of 20 but the spectrum remains very hard. Such extremely hard X-ray spectra and variability have certainly been seen in other young stars (e.g., W51 IRS2E in MOXC1 and two early-B stars in IRAS 20126+4104, Anderson et al. 2011), but this is still an exceptional source due to its high luminosity and extreme variability; it deserves further attention.

\subsubsection{HII 351.2+0.5}

East of GUM 61 lies another prominent Hir region south of NGC 6334's main ridge, called HiI $351.2+0.5$ by Russeil et al. (2016) (Figure 5(a)). Comparatively few X-ray point sources are found in this part of our ACIS mosaic, even though it is captured by three ACIS pointings, because it is only observed far off-axis in each of these pointings. This region does serve to illustrate the usefulness of including off-axis CCDs in our mosaics; diffuse X-ray emission is seen throughout this area. It appears at the center of Hir $351.2+0.5$ and at the bubble rim, perhaps indicating hot plasma flowing out its southeast side.

The brightest X-ray source in this part of the ACIS mosaic is c6709 (CXOU J172052.63-360420.6); it is the X-ray counterpart to the massive binary HD 156738 (Sana et al. 2014), spectral type O6III((f)) (Sota et al. 2014), located near the center of Hir 351.2+0.5. Source c6709 has 948 net counts and a median energy of $1.2 \mathrm{keV}$. As for ACIS source c1934 above, c6709 requires a two-temperature fit, TBabs(apec1 + apec2), although the plasmas are much softer here. Fit parameters are $N_{H}=$ $1.4 \times 10^{22} \mathrm{~cm}^{-2}, k T 1=0.2 \mathrm{keV}, k T 2=0.5 \mathrm{keV}$, and $L_{X}=5.5 \times 10^{32} \mathrm{erg} \mathrm{s}^{-1}$. These soft plasmas are consistent with instability-driven wind shocks in the individual stars.

\subsubsection{SNR G351.2+0.1}

Two archival ACIS-S observations of SNR G351.2+0.1 are included in our mosaic, totaling 53 ks exposure. This composite SNR sits to the southeast of NGC 6334 and was observed by Chandra to search for its neutron star and pulsar wind nebula; no clear detection was made. Radio observations 

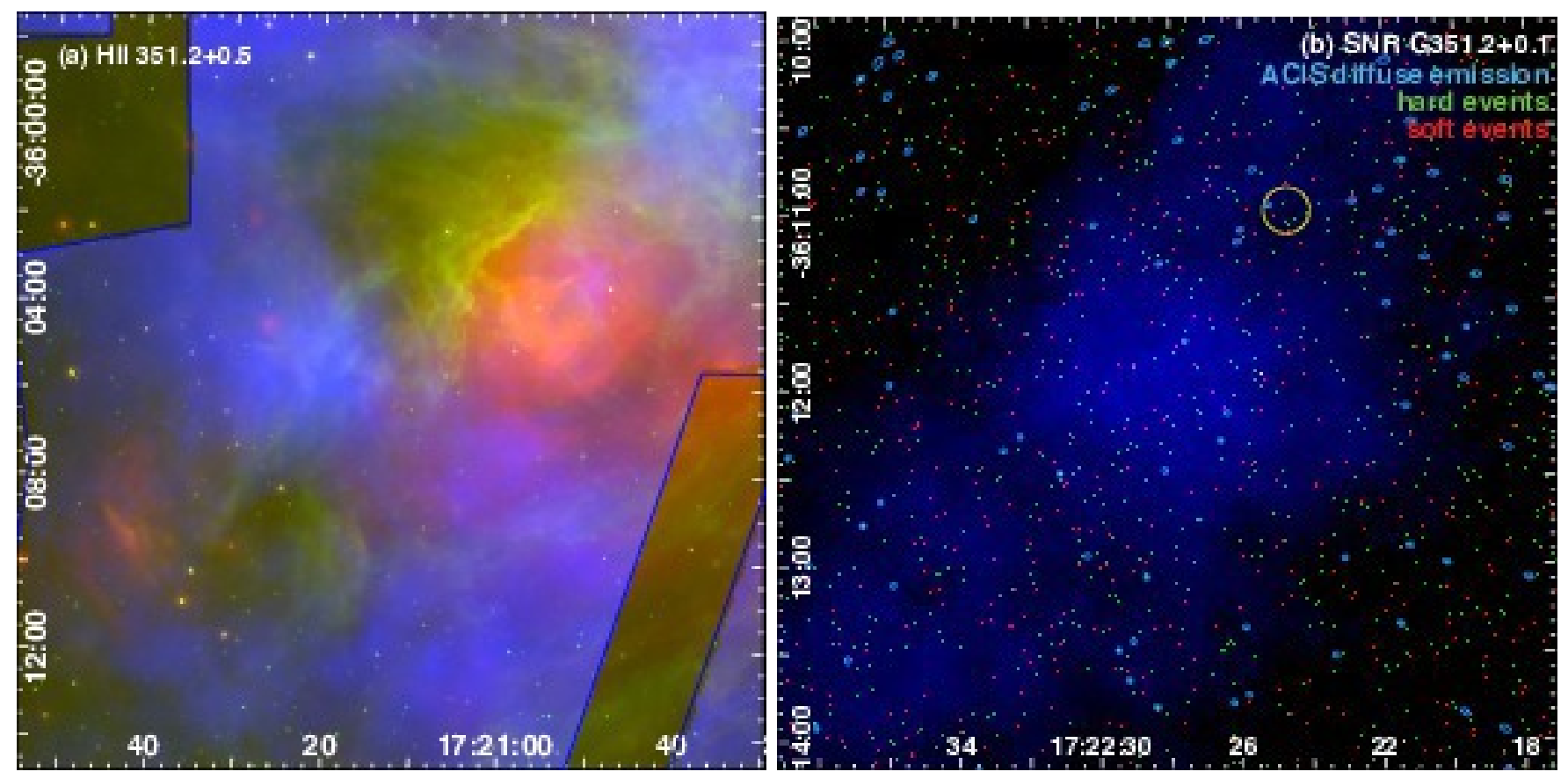

Figure 5. NGC 6334 southeast. (a) Zoomed version of Figure 1(b) for Hir 351.2+0.5. (b) ACIS event data and diffuse emission for the SNR G351.2+0.1 region. A yellow circle indicates the central radio object (Becker \& Helfand 1988).

show a $4^{\prime} \times 6^{\prime}$ shell, flattened along the northern side, with a $16^{\prime \prime}$-diameter central object (Becker \& Helfand 1988) (shown in (Figure 5(b)). Dubner et al. (1993) note that the flattened northern edge of the remnant may indicate that it is encountering dense molecular material that is slowing its expansion in this direction. Perhaps this is an indication that the SNR is associated with the G352 GMC, although the SNR distance is not well-determined.

There is no bright ACIS source that is clearly the counterpart of the central radio object. The wider field, however, shows a large number of X-ray sources concentrated in the vicinity of the SNR (Figure 1(a)). Perhaps these constitute another young cluster; this should be investigated at longer wavelengths. Excising those point sources leaves some faint, soft diffuse X-ray emission apparent in Figure 5(b); this may be the feeble X-ray signature of the SNR. A large region surrounding this faint diffuse emission is conspicuously lacking in diffuse X-rays (Figure 1(b)); cold material in the G352 GMC may be shadowing any hot plasma generated by the SNR or NGC 6334 there. 


\section{2. $W 75 N$}

W75N is part of the great concentration of star formation in the Cygnus X North molecular cloud complex (Reipurth \& Schneider 2008). It contains several UCHıRs ionized by early-B stars (Shepherd et al. 2004) and exhibits complicated dynamics from multiple molecular outflows (Shepherd et al. 2003; Davis et al. 2007). Persi et al. (2006) find at least 25 cluster members based on their IR excess; we find that several of these have X-ray counterparts. Kumar et al. (2007), also using near- and mid-IR data, find a distributed population of young stars across the region, tracing the molecular filaments.

The single ACIS observation of W75N (Figure 6) shows a concentration of hard sources near the aimpoint. ACIS source c202 (CXOU J203836.47+423733.7) appears to be the X-ray counterpart to VLA_3 (Bb), an UCHıR with Lyman continuum flux consistent with ionization by a B1 star (Shepherd et al. 2004). Source c202 has only five counts, but its median energy is $6.7 \mathrm{keV}$ and all five counts have energies $>6 \mathrm{keV}$. The spectral fit gives $N_{H} \sim 30 \times 10^{22} \mathrm{~cm}^{-2}, k T \sim 7 \mathrm{keV}$, and $L_{X} \sim 8 \times 10^{30} \mathrm{erg} \mathrm{s}^{-1}$ but the fit parameters are not well-constrained. This is just a rough characterization given the limited counts, but there is no doubt that this is an extreme X-ray source, exceptionally hard and highly obscured; such emission is not expected from a single B1 star.

Diffuse X-ray emission is seen on the west side of W75N despite the high obscuration and short $(<30 \mathrm{ks})$ observation. It is brightest in the southwest corner of the ACIS-I image, outside the large dark arc in the Spitzer images that runs vertically at RA 20:38:15. In Figure 6(b) we have scaled the ACIS image to show the fainter diffuse emission surrounding the central cluster and filling apparent cavities in the Spitzer emission. Bright diffuse emission is seen far off-axis on the ACIS-S CCDs; this may be associated with the wider Cygnus X star-forming complex. The central region also shows extensive diffuse X-ray emission (Figure 6(c)) extending all the way down to the cluster center (Figure 6(d)). 


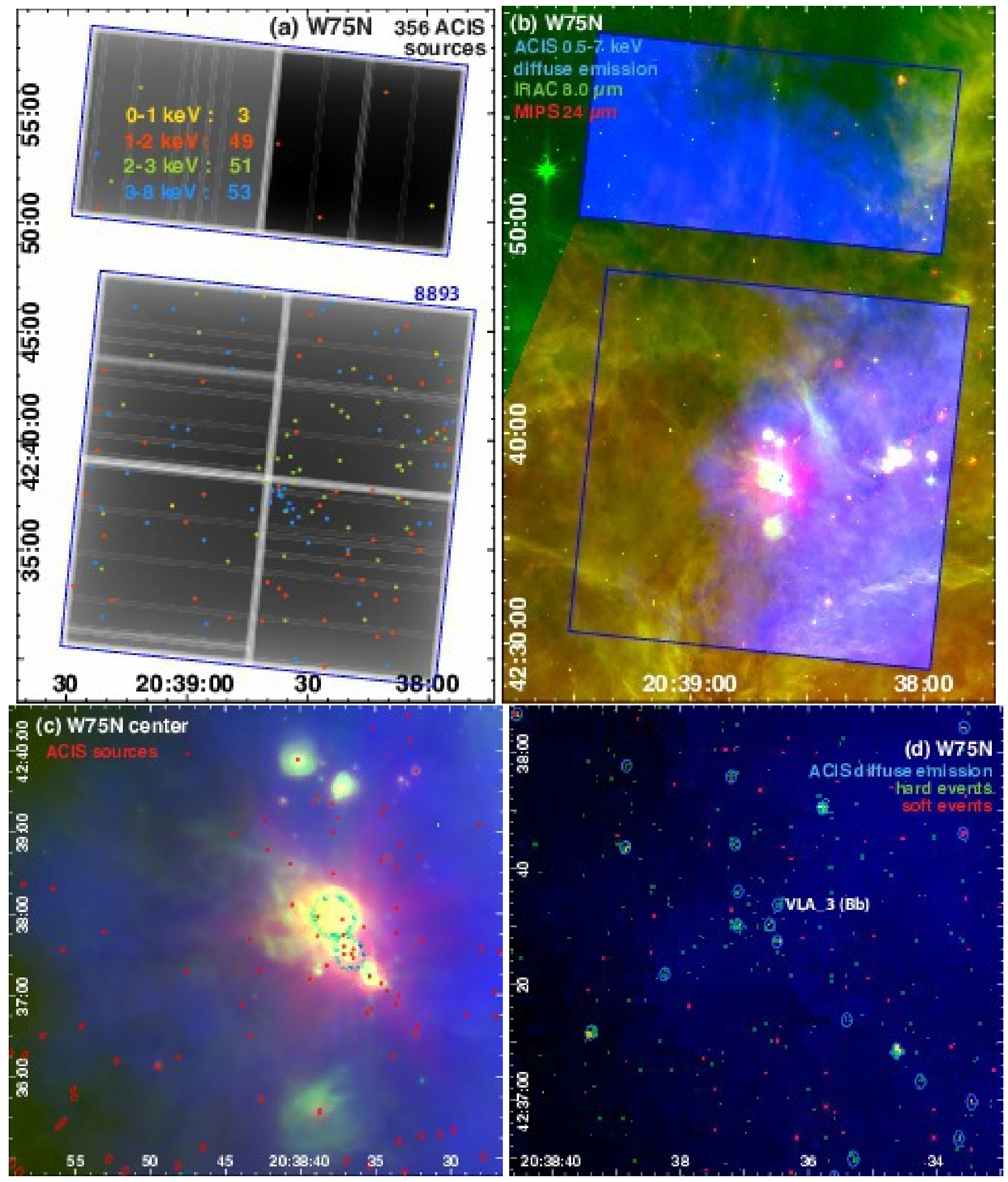

Figure 6. W75N. (a) ACIS exposure map with brighter ( $\geq 5$ net counts) ACIS point sources overlaid; colors denote median energy for each source. The ObsID number is shown in blue. (b) ACIS diffuse emission in the Spitzer context. (c) Zoom of (b) showing the center of W75N and surrounding diffuse X-ray emission. Green rings are artifacts due to MIPS saturation. Red polygons show extraction regions for ACIS sources. (d) ACIS event data and diffuse emission at the center of W75N. The hard X-ray counterpart to the radio UCHitR VLA_3 $(\mathrm{Bb})$ is marked. 


\section{3. $R C W 120$}

The innocuous visual Hir region RCW 120 was catapulted to fame by the Spitzer GLIMPSE survey (Churchwell et al. 2006; Zavagno et al. 2007), which revealed it to be a canonical example of a single, dusty bubble hosting massive star formation (Deharveng \& Zavagno 2008). It has since received much attention at long wavelengths, especially from Herschel, which detects several very young stars forming in dense, cold material around the edge of the bubble (Zavagno et al. 2010; Figueira et al. 2017). The bubble appears to be disrupted at its northern edge and is leaking photons at several points around its rim (Zavagno et al. 2007; Deharveng et al. 2009; Anderson et al. 2015). Such "leaky" Hir regions may demonstrate that ionizing radiation from massive stars extends well beyond classical His region boundaries, influencing star formation over a much wider area (Zavagno et al. 2007).

The Chandra observations of RCW 120 were presented in the SFiNCs (Star Formation in Nearby Clouds) survey (Getman et al. 2017). SFiNCs studies X-ray point sources in Chandra observations of nearby, typically lower-mass star-forming regions, so this is the only target that MOXC2 and SFiNCs have in common. We included RCW 120 in MOXC2 (Figure 7) primarily to facilitate study of the diffuse X-ray emission in this iconic Spitzer bubble, but this common target does give us the opportunity to compare our point source catalog with that of SFiNCs. This is a useful check, since the two projects use similar (but not identical) analysis methods. The SFiNCs analysis is a few years older than the MOXC2 analysis.

In MOXC2, we find 999 X-ray sources in the main ACIS-I array observations of RCW 120 itself; SFiNCs found 678 X-ray sources in the same data. Matching the two catalogs, we find 604 formal matches, 395 MOCX2 sources not matched, and 74 SFiNCs sources not matched. One goal of MOXC2 is to detect fainter sources, thus we expect to have many unmatched MOXC2 sources. In several cases, either MOXC2 or SFiNCs found a pair of sources while the other catalog found a single source. Many of the unmatched sources (in both catalogs) are far off-axis and faint. For such sources, the source validity metric ${ }^{20}$ can be sensitive to slight source position differences, algorithmic changes to the background calculation, and differences in the catalogs (neighboring sources affect the background calculation). Because source candidates are pruned by a simple threshold on this noisy validity metric, two reductions of the same data will not produce identical catalogs near the detection limit.

For our ACIS mosaic of the RCW 120 region, we included neighboring observations of SNRs to get a wider context for RCW 120's diffuse X-ray emission: ObsID 6721 on CTB 37A (Pannuti et al. 2014) and ObsID 6692 on CTB 37B (Aharonian et al. 2008) (Figure 7(a)). Large masks were applied to these SNRs to keep them from dominating our smoothed diffuse X-ray image of the mosaic (Figure $7(\mathrm{~b})$ ). For completeness, we include the point sources we found in these fields in our X-ray source list for RCW 120.

Extensive diffuse X-ray emission is seen in RCW 120 (Figure 7(c)). It appears to trace hot plasma through a breach in the northeast side of the bubble and out into the surrounding ISM. The whole bubble apparently shadows background diffuse X-ray emission.

Clumps of X-ray point sources are shown in Figures 7(d)-(f). Panels (d) and (f) show groups of sources on the bubble rim; panel (e) shows the central cluster. The main ionizing source for RCW 120

\footnotetext{
20 The source validity metric is a $p$-value for the no-source ("null") hypothesis, i.e., that all extracted counts are background.
} 


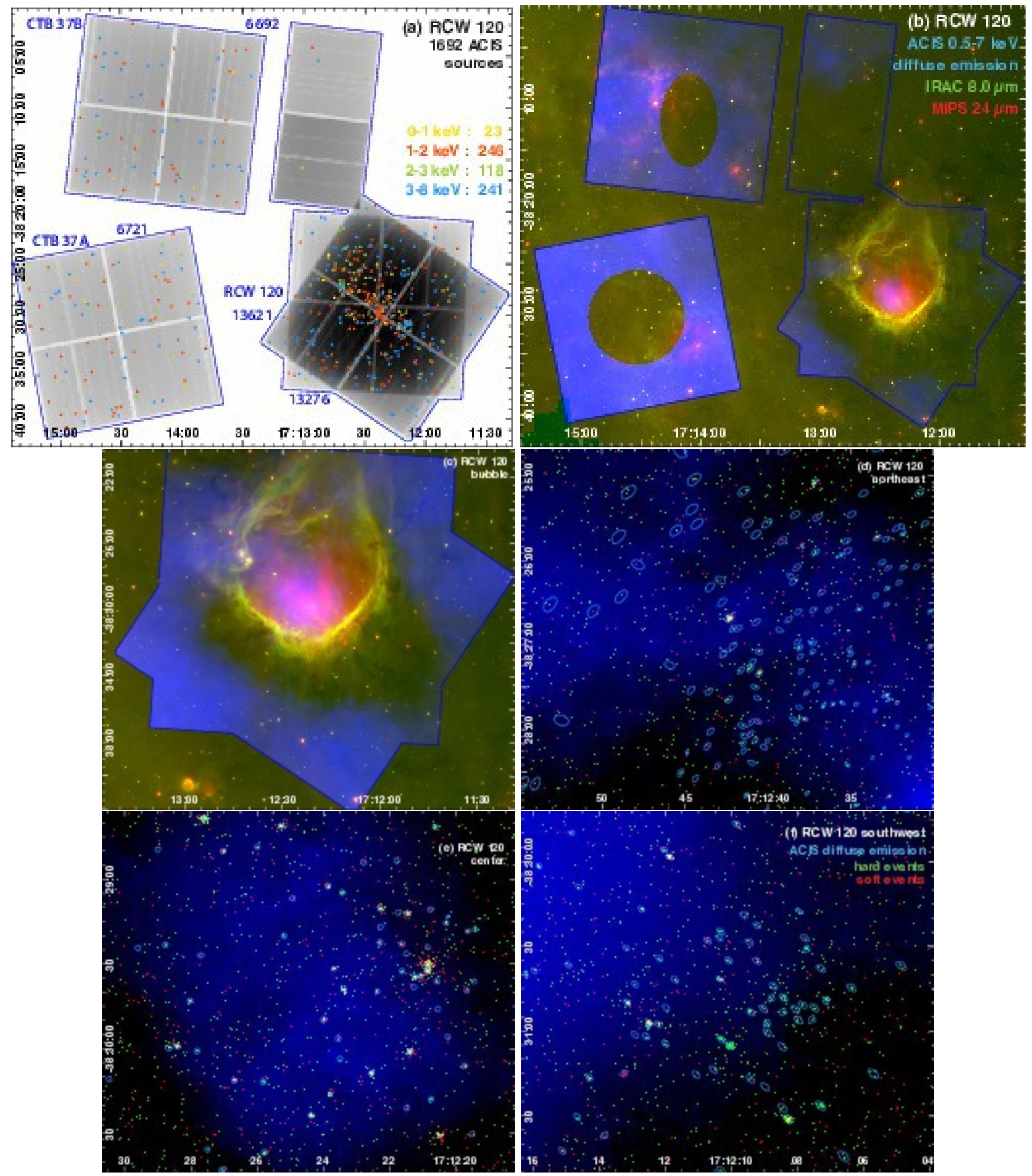

Figure 7. RCW 120. (a) ACIS exposure map with brighter ( $\geq 5$ net counts) ACIS point sources overlaid; colors denote median energy for each source. ObsID numbers and regions named in the text are shown in blue. (b) ACIS diffuse emission in the Spitzer context. (c) Zoomed version of (b) for the RCW 120 bubble. (d)-(f) ACIS event data and diffuse emission detailing clumps of X-ray point sources in RCW 120. (d) On the northeast rim of the bubble, where diffuse X-ray emission appears to breach the bubble wall. (e) In the south-central part of the bubble, associated with the brightest diffuse X-ray emission in the bubble. The ionizing $\mathrm{O} 8$ star is the bright X-ray source on the western side of this group. (f) On the southwest rim of the bubble. 
is LSS 3959 (CD-28 11636 in SIMBAD), an O8V star (Zavagno et al. 2007). Its X-ray counterpart is ACIS source c1121 (CXOU J171220.84-382930.3, prominent in Figure 7(e)), with 682 net counts and a median energy of $1.4 \mathrm{keV}$. The spectral fit requires two thermal plasma components, TBabs(apec1 + apec2); the softer plasma dominates the spectrum. Parameters are $N_{H}=1.4 \times 10^{22} \mathrm{~cm}^{-2}, k T 1=$ $0.5 \mathrm{keV}, k T 2=2 \mathrm{keV}$, and $L_{X}=1.4 \times 10^{32} \mathrm{erg} \mathrm{s}^{-1}$. The harder plasma component is not expected from a single late-O star and is perhaps suggesting magnetic activity or binarity.

\subsection{IRAS 20126+4104}

Like W75N described above, the IRAS 20126+4104 MSFR is another component of the extensive and diverse star formation complex in Cygnus (Reipurth \& Schneider 2008). Its distance is welldetermined by maser parallax (Moscadelli et al. 2011). IRAS 20126+4104 itself is a famous massive young stellar object (MYSO) with a prominent molecular outflow that has long been the subject of radio study (e.g., Cesaroni et al. 2005). Cesaroni et al. (2014) have imaged this MYSO at $1.4 \mathrm{~mm}$, finding a distorted disk and associated jet rotating around a proto-B star. De Buizer et al. (2017) presents a recent SOFIA detection of the MYSO; he finds that SOFIA and Herschel data show elongation in the direction of the outflow. The Chandra data were first presented by Anderson et al. (2011), with details on the X-ray counterparts to radio sources I20S and I20Var (Hofner et al. 2007). These authors note the detection of 150 point sources in the Chandra data and suggest that IRAS $20126+4104$ is surrounded by a young stellar cluster. Those sources are catalogued in a recent paper by the same group (Montes et al. 2015), who determine that 80 of the $150 \mathrm{X}$-ray sources are likely members of this MSFR. They find that the surface density of X-ray sources peaks on the MYSO, thus IRAS $20126+4104$ is a young cluster forming an early-B star at its center.

Our analysis of the same Chandra data found more faint point sources, as designed (Figure 8); we recovered all 150 X-ray sources reported by Montes et al. (2015). Many X-ray sources near the center of the cluster resolve into close pairs in our analysis (Figure 8(d)). Anderson et al. (2011) described the exceptional X-ray spectra of the ACIS counterparts to I20S and I20Var; we find similar spectral fit results. They concluded that I20S was an early-B MYSO and I20Var a likely IMPS. We concur with these findings and emphasize their implications: in some cases, stars that will become $\mathrm{X}$-ray-quiet on the main sequence are extraordinarily hard, bright X-ray emitters in their youth, perhaps due to magnetic activity similar to that found in lower-mass pre-MS stars (Povich et al. 2011; Gregory et al. 2016).

We find prominent diffuse X-ray emission in this ACIS field (Figure 8(b)-(d)), with a bright ridge running the length of the ACIS-I array to the east of the cluster (Figure 8(b)). The image center shows an arc of X-ray emission just to the east of the large pillar that hosts the embedded cluster (Figure 8(c)); between this and the eastern ridge is an absence of diffuse X-rays coincident with faint WISE $22 \mu \mathrm{m}$ emission. This may be material shadowing the diffuse X-rays. Faint diffuse X-ray emission pervades the pillar as far west as the central cluster (Figure 8(d)), then drops off sharply. The western side of the ACIS-I field shows a complex mix of fainter diffuse X-ray emission anticoincident with bright WISE structures; this fades to only minimal diffuse X-ray emission on the off-axis ACIS-S CCDs. Again shadowing or displacement of hot gas is likely here. 

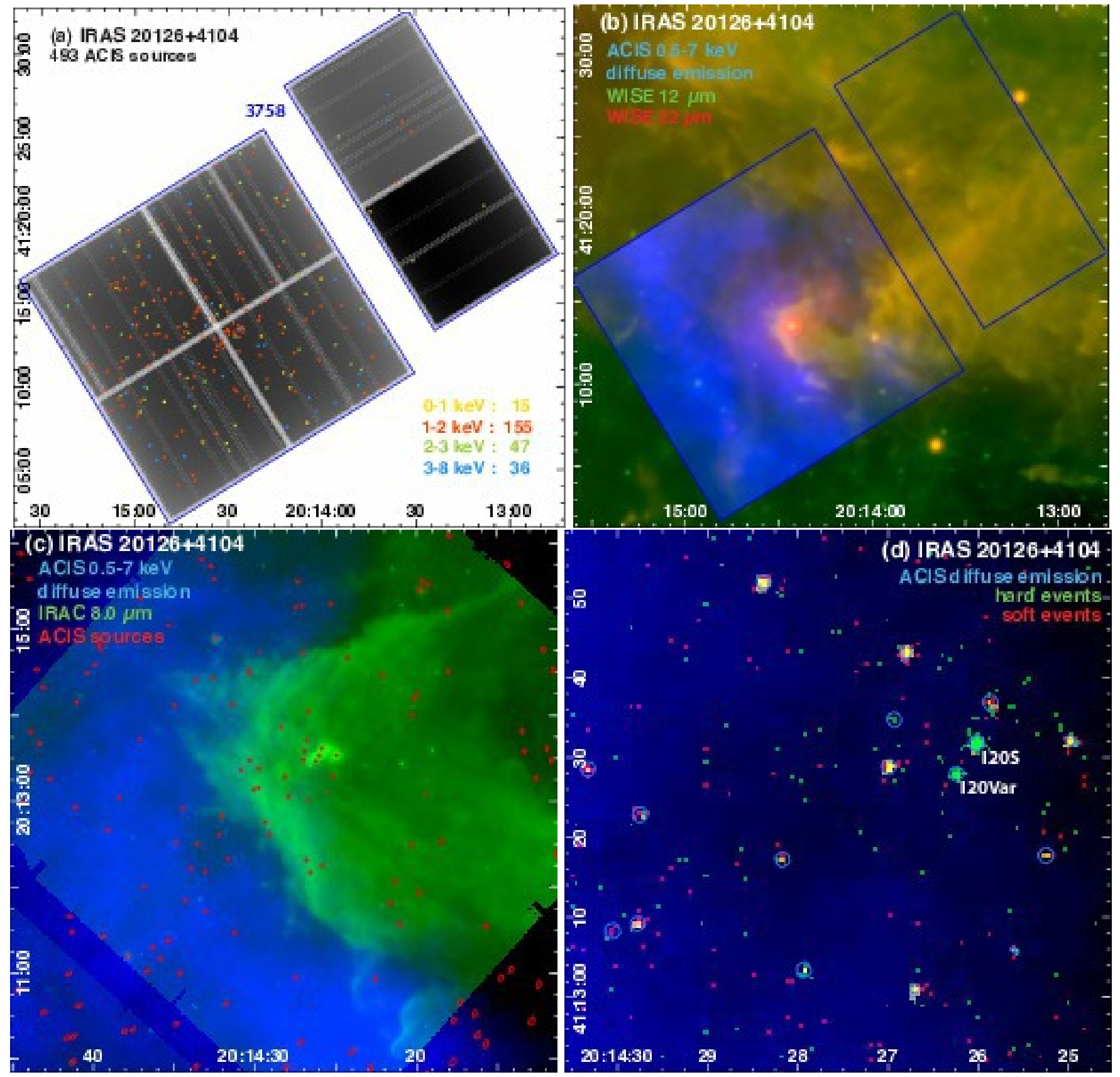

Figure 8. IRAS 20126+4104. (a) ACIS exposure map with brighter ( $\geq 5$ net counts) ACIS point sources overlaid; colors denote median energy for each source. The ObsID number is shown in blue. (b) ACIS diffuse emission in the WISE context. Spitzer data lack full field coverage for this target. (c) ACIS diffuse emission around the main pillar of IRAS 20126+4104, shown with Spitzer/IRAC data (badly saturated MIPS data are omitted). Red polygons show extraction regions for ACIS sources. (d) ACIS event data and diffuse emission at the center of IRAS 20126+4104. Zoom to see close pairs of X-ray sources. Hard X-ray counterparts to radio sources I20S and I20Var are marked. 


\section{5. $W 31 N$}

The vicinity of the famous giant HII region W31 has several Chandra/ACIS observations of a variety of targets at a wide range of distances. As for other MOXC2 targets, we have combined them into a wide-field mosaic (Figures 9(a) and (b)) to sample the X-ray point source populations and diffuse emission present in large Galactic Plane fields.

Deharveng et al. (2015) showed that the northern component of the W31 complex (W31N, which they call G010.32-0.15) is actually a foreground MSFR unassociated with the other major MSFRs along this sightline, at a distance of just $1.75 \mathrm{kpc}$. W31N (G10.3-0.1) is a bipolar HII region ionized by an O5-O6 star (Bik et al. 2005) and containing an IR cluster; it is triggering a menagerie of second-generation massive stars and their UCHıRs in the dense filament at its waist (Dewangan et al. 2015; Deharveng et al. 2015).

At this modest distance, it makes a rich Chandra target; we find $>50$ X-ray point sources in the central cluster and 490 sources across the ACIS-I field centered on W31N. As demonstrated below, with these data we can study both the first-generation ionizing cluster and the onset of X-ray emission in massive stars just formed. Figure 9(c) shows that W31N also contains diffuse X-ray emission and strongly shadows (or displaces) the diffuse emission seen in the wider W31 field.

The original $6.5 \mathrm{ks}$ ACIS-S observation (ObsID 10518) of W31N detected the ionizing O5 star (source ChI J180857-2004_2 in Anderson et al. 2014); these authors suggest that it could be a CWB. In our data (Figure 9(d)), this is source c1345 (CXOU J180859.12-200508.4), with 553 net counts and a median energy of $3.3 \mathrm{keV}$. A spectral fit gives $N_{H}=4.9 \times 10^{22} \mathrm{~cm}^{-2}, k T=3.8 \mathrm{keV}$, and $L_{X}=2.4 \times 10^{32} \mathrm{erg} \mathrm{s}^{-1}$. Once again, this high column could be hiding an additional soft plasma component, so this luminosity is a lower limit. We concur with Anderson et al. (2014); this hard spectrum suggests a CWB.

Our source c1447 (CXOU J180901.48-200507.5) is the MYSO (YSO \#4) in clump C2 of Deharveng et al. (2015). It has 43 net counts, a median energy of $4.6 \mathrm{keV}$, and is variable; fit parameters are $N_{H}=21 \times 10^{22} \mathrm{~cm}^{-2}, k T=5.5 \mathrm{keV}$, and $L_{X}=5 \times 10^{31} \mathrm{erg} \mathrm{s}^{-1}$. This extremely high column explains the high median energy. The hard thermal plasma and variability demonstrate that dramatic X-ray emission turns on early in the formation process of at least some massive stars.

Near the center of our ACIS mosaic of the W31 complex (Figure 9(b)), the conspicuous MSFR not yet observed by Chandra is G10.2-0.3, a giant Hiı region at a distance of $3.4 \mathrm{kpc}$ (Blum et al. 2001). It contains four O stars and four MYSOs in a very young (0.6 Myr) cluster (Furness et al. 2010), several UCHıRs (Ghosh et al. 1989), and XMM source detections (Nebot Gómez-Morán et al. 2015); it would make an excellent Chandra target. Southwest of G10.2-0.3, our mosaic includes the piled-up soft gamma repeater SGR 1806-20; we have masked its bright X-ray halo of dust-scattered light (Kaplan et al. 2002) in our image of diffuse X-ray emission.

At the southeast corner of our ACIS mosaic, we have included a 9.9-ks ACIS-I observation of the radio-bright SNR G9.95-0.81 (Brogan et al. 2006) (ObsID 10713). This field also includes the large GLIMPSE bubble CN139, with a near kinematic distance of $4.3 \mathrm{kpc}$ (Churchwell et al. 2007; Watson et al. 2009). Quite surprisingly (given the short observation), Figure 9(e) clearly shows diffuse X-ray emission coincident with this bubble, somewhat offset to the northwest from the center of the radio SNR. Spectral analysis to determine the absorbing column to this X-ray emission should help to determine whether it is really associated with the bubble. 


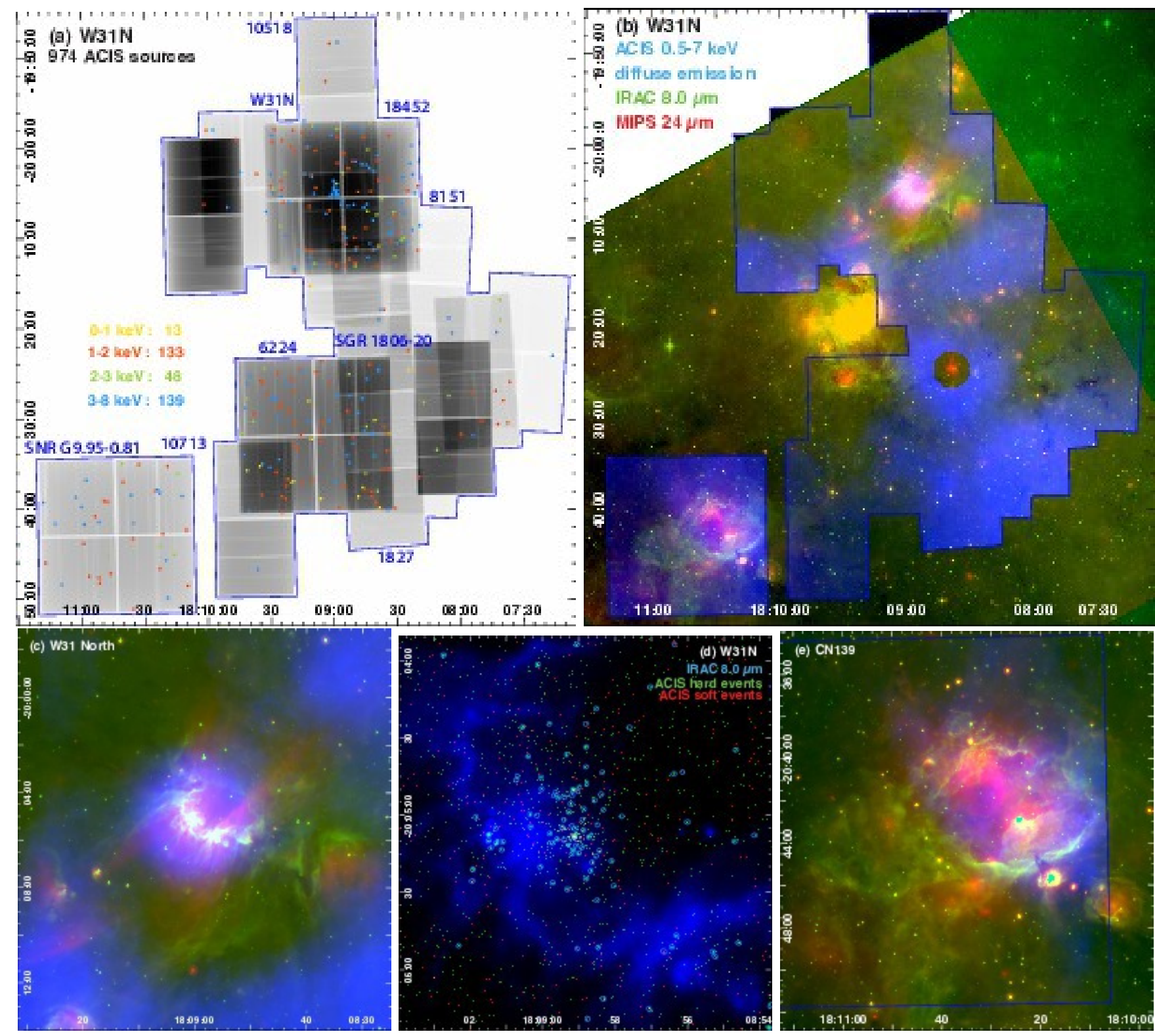

Figure 9. W31. (a) ACIS exposure map with brighter ( $\geq 5$ net counts) ACIS point sources overlaid; colors denote median energy for each source. ObsID numbers and regions named in the text are shown in blue. (b) A wide-field ACIS mosaic of the W31 region, featuring ACIS diffuse emission in the Spitzer context. A region around the bright X-ray source SGR 1806-20 has been masked so that fainter diffuse X-ray emission across the field can be displayed. (c) Zoomed version of (b) for the nearby MSFR W31N. (d) ACIS event data on W31N. For context, Spitzer/IRAC $8 \mu \mathrm{m}$ emission is shown in blue. (e) Zoomed version of (b) for the GLIMPSE bubble CN139 at the southeast corner of our W31 ACIS mosaic.

Through further serendipity, the globular cluster 2MASS GC02 is captured far off-axis on the S3 CCD in ObsID 10713, visible (by zooming) at the bottom of the Spitzer/IRAC $8 \mu$ m image in Figure 9(b). ACIS source c2200 (CXOU J180936.55-204645.1) is centered on 2MASS GC02. It has 38 net counts and a median energy of $4.0 \mathrm{keV}$. It is most likely a composite of emission from multiple members of the globular cluster, but its properties might help to establish the feasibility for a longer ACIS observation of this target. 


\subsection{IRAS $19410+2336$ and $N G C 6823$}

Starting with a 20-ks observation of the MSFR IRAS 19410+2336, we built a 4-pointing ACIS-S mosaic of overlapping short observations from archival Chandra data in this region (Figures 10(a) and (b)). This is the shallowest mosaic in MOXC2 (just 2-20 ks). This mosaic also features a second MSFR, NGC 6823, presumably not associated with IRAS 19410+2336 although at a similar distance. This is the first of three examples of ACIS mosaics in MOXC2 that feature two unrelated MSFRs that just happen to be projected near each other on the sky.

IRAS $19410+2336$ is a young, compact, embedded MSFR made up of two main star-forming clumps (Beuther et al. 2002; Rodón et al. 2012), both exhibiting masers (Beuther et al. 2002) and a large number of outflows (Beuther et al. 2003). The Chandra observation of IRAS 19410+2336 (ObsID 1868, $20 \mathrm{ks}$ ) was analyzed by Beuther et al. (2002), who tabulated 13 X-ray sources in the vicinity of the two clumps (Figure 10(d)). They find these sources to have hard X-ray spectra and to be heavily obscured, with fairly high intrinsic luminosities. They conclude that the X-ray sources are mainly IMPS; as noted above, subsequent work has confirmed that such sources can be strong X-ray emitters (Povich et al. 2011; Gregory et al. 2016).

Beuther et al. (2002) included the full $20 \mathrm{ks}$ exposure in their analysis; our standard processing of this dataset leaves just $8.7 \mathrm{ks}$ of usable data on the aimpoint CCD S3 and on S1, the other backsideilluminated device, due to space weather resulting in high backgrounds for these chips. The ACIS frontside-illuminated devices in operation for this observation (I2, I3, S2, and S4) were unaffected by these background flares, so they retain the full integration time in our analysis. Unfortunately, the two main embedded clumps in IRAS $19410+2336$ are imaged on CCD S3, so we report source detections in only $8.7 \mathrm{ks}$ of data for these clumps. As we will see below, this is comparable to the ACIS integration time we have on the second MSFR imaged in this multi-pointing ACIS-S mosaic, NGC 6823.

As shown in Figures 10(c) and (d), we find X-ray sources in the same regions as Beuther et al. (2002), although not always the same sources. The southern clump is well-populated with X-ray sources but the northern one is not; rather a second group of X-ray sources sits to the east of the northern clump. Diffuse X-ray emission appears between the two groups of X-ray sources.

About $26^{\prime}$ south of IRAS $19410+2336$ we find the other MSFR in our mosaic, NGC 6823 (Prato et al. 2008), captured on two short ACIS-S observations (Figure 10(e)). It features a tight grouping of massive stars at its center (Riaz et al. 2012; Shi \& Hu 1999), with pre-MS stars distributed out to the cluster radius of $16.2^{\prime}$ (Kharchenko et al. 2005). Its earliest star is HDE 344784 A, with a spectral type of O6.5 V((f))z (Arias et al. 2016). Anderson et al. (2014) detect its X-ray counterpart as their source ChI J194310+2318_5. In our analysis, this is Chandra source c228 (CXOU J194310.96+231745.5), with 72 net counts and a median energy of $1.1 \mathrm{keV}$. Our simple thermal plasma fit yields $N_{H}=$ $0.9 \times 10^{22} \mathrm{~cm}^{-2}, k T=0.6 \mathrm{keV}$, and $L_{X}=1.9 \times 10^{32} \mathrm{erg} \mathrm{s}^{-1}$.

Anderson et al. (2014) list several other X-ray sources in this region; we also find many other sources. Despite having just 7.9 ks total integration on this target and with the cluster center placed 8 ' off-axis on the 6-ks ObsID, ACIS still clearly detects this MSFR. Several of our X-ray sources are counterparts to young stellar objects tabulated in Riaz et al. (2012).

In the fourth pointing of this mosaic (ObsID 8164, $2.7 \mathrm{ks}$ ) we have two sources at the aimpoint, the piled-up source c19 (CXOU J194154.60+225112.3) and its close neighbor c20 (CXOU J194154.60+225112.8). Due to crowding, extraction apertures are reduced to $45 \%$ for 


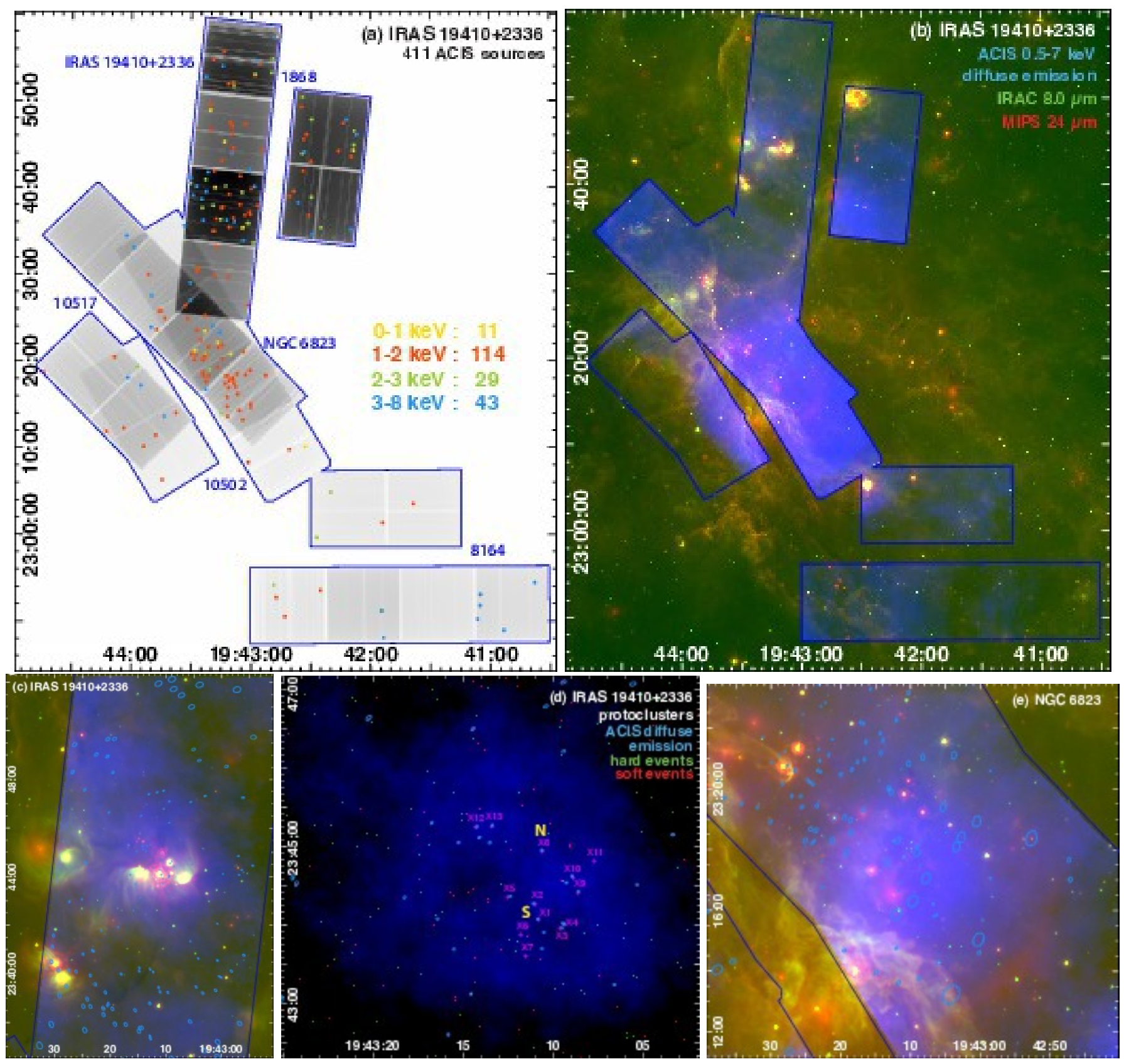

Figure 10. IRAS $19410+2336$. (a) ACIS exposure map with brighter ( $\geq 5$ net counts) ACIS point sources overlaid; colors denote median energy for each source. ObsID numbers and regions named in the text are shown in blue. (b) ACIS diffuse emission in the Spitzer context. (c) Zoomed version of (b) for IRAS 19410+2336, with MOXC2 point source extraction regions overlaid. (d) ACIS event data and diffuse emission at the center of IRAS 19410+2336. MOXC2 point source extraction regions are blue; X-ray sources from Beuther et al. (2002) are noted in magenta. Approximate centers of the northern and southern starforming clumps (Rodón et al. 2012) are marked with N and S respectively. (e) Zoomed version of (b) for NGC 6823, with MOXC2 point source extraction regions overlaid.

c19 (25 net counts, median energy $3.3 \mathrm{keV}$ ) and just 36\% for c20 (10 net counts, median energy $2.4 \mathrm{keV})$. With so few counts, we cannot correct c19 for pile-up. Rough spectral fits to these sources show them both to be very hard $(\mathrm{kT} \sim 10 \mathrm{keV})$ but with very different absorbing columns and 
absorption-corrected full-band fluxes: $N_{H}=4 \times 10^{22} \mathrm{~cm}^{-2}, F_{X} \sim 8 \times 10^{-13} \mathrm{erg} \mathrm{s}^{-1} \mathrm{~cm}^{-2}$ for c19 and $N_{H}=1 \times 10^{22} \mathrm{~cm}^{-2}, F_{X} \sim 2 \times 10^{-13} \mathrm{erg} \mathrm{s}^{-1} \mathrm{~cm}^{-2}$ for $\mathrm{c} 20$. Anderson et al. (2014) find a single X-ray source (ChI J194152+2251_2) at this location (a blend of our c19 and c20), using this same dataset; they fit it with a power law with $N_{H}=0.9 \times 10^{22} \mathrm{~cm}^{-2}$, slope $\Gamma \sim 0.4$, and absorption-corrected full-band flux $F_{X} \sim 7 \times 10^{-13} \mathrm{erg} \mathrm{s}^{-1} \mathrm{~cm}^{-2}$. They noted that the near-IR image of this source appears extended, consistent with a blend of sources.

Despite the very short integration times, diffuse X-ray emission is seen throughout this mosaic. It is brightest around NGC 6823 (Figure 10(e)), likely indicating a mix of unresolved pre-MS stars in the cluster and perhaps hot plasma from the winds of the O6 star. We see highly structured diffuse emission suffusing the deeply embedded IRAS 19410+2336 (Figure 10(d)) and pervading the surrounding field (Figure 10(c)). The wider field contains SNR G59.5+0.1 (Xu \& Wang 2012), which may also be contributing to diffuse X-ray emission in our mosaic.

\subsection{W42 and $R S G C 1$}

In a recent Chandra GO+GTO program, we targeted the newly-formed MSFRs W42 and W33, both offering rich samples of very young $(<0.2 \mathrm{Myr})$ massive stars ionizing UCHIIRs, to study the emergence of X-ray emission in these objects and their influence on the early evolution of MSFRs. At the same time we explored two of the Galaxy's extremely rare YMCs, with $>10^{4} \mathrm{M}_{\odot}$ (Portegies Zwart et al. 2010), Red Supergiant Cluster 1 (RSGC1), age 10 Myr (Froebrich \& Scholz 2013), and Cl 1813-178, age 4.5 Myr (Messineo et al. 2011). YMCs persist for $>10$ Myr because of their extraordinarily large masses; they allow us to explore changes in X-ray emission from massive stars as they approach the ends of their lives, along with the neutron stars and SNRs that they leave behind.

Here our ACIS-I mosaic (Figure 11) captures both the nearby MSFR W42 (Blum et al. 2000) and the unrelated background YMC RSGC1 (Figer et al. 2006), along with two pulsars and their PWNe (Gotthelf \& Halpern 2008). We recover all X-ray sources tabulated by Gotthelf \& Halpern (2008). We find a dense cluster of X-ray sources at the center of W42 (Figure 11(c)).

W42 hosts at least 4 UCHıRs (Urquhart et al. 2013) and contains over 500 candidate young stellar objects based on IR-excesses (Dewangan et al. 2015). The brightest near-IR source, W42 No. 1, is an O5-O6 star (Blum et al. 2000). W42's edge-on orientation illustrates the connection between molecular filaments and MSFRs: part of a massive filament has collapsed to form the MSFR that is now blowing a bipolar bubble normal to the filament's long axis (Deharveng et al. 2010; Dewangan et al. 2015). We find few X-ray sources along this filament south of the W42 cluster.

The bright yellow X-ray source slightly northeast of field center in Figure 11(c) is c1575 (CXOU J183815.27-064758.8), the counterpart to W42 No. 1 (Blum et al. 2000). It has 148 net counts and a median energy of $2.4 \mathrm{keV}$; the spectral fit gives $N_{H}=2.3 \times 10^{22} \mathrm{~cm}^{-2}, k T=3.3 \mathrm{keV}$, and $L_{X}=5.5 \times 10^{31} \mathrm{erg} \mathrm{s}^{-1}$. Again this thermal plasma temperature is too hard for a single mid-O star, although the luminosity is modest compared to many CWBs. Alternatively, the W42 complex may be more distant than the $2.2 \mathrm{kpc}$ (Blum et al. 2000) we have assumed; Dewangan et al. (2015) use a distance of $3.8 \mathrm{kpc}$. That distance increases the X-ray luminosity of source $\mathrm{c} 1575$ to $L_{X}=1.6 \times 10^{32} \mathrm{erg} \mathrm{s}^{-1}$.

RSGC1 (Figer et al. 2006) is a 10-Myr-old obscured cluster (Froebrich \& Scholz 2013; Davies et al. 2008), the fifth most massive Galactic YMC known (Richards et al. 2012). It has > 200 massive stars, far more than the other known RSGCs (Froebrich \& Scholz 2013), that form a compact cluster. The X-ray-bright pulsar AX J1838.0-0655 is thought to be a member of RSGC1 (Gotthelf \& Halpern 

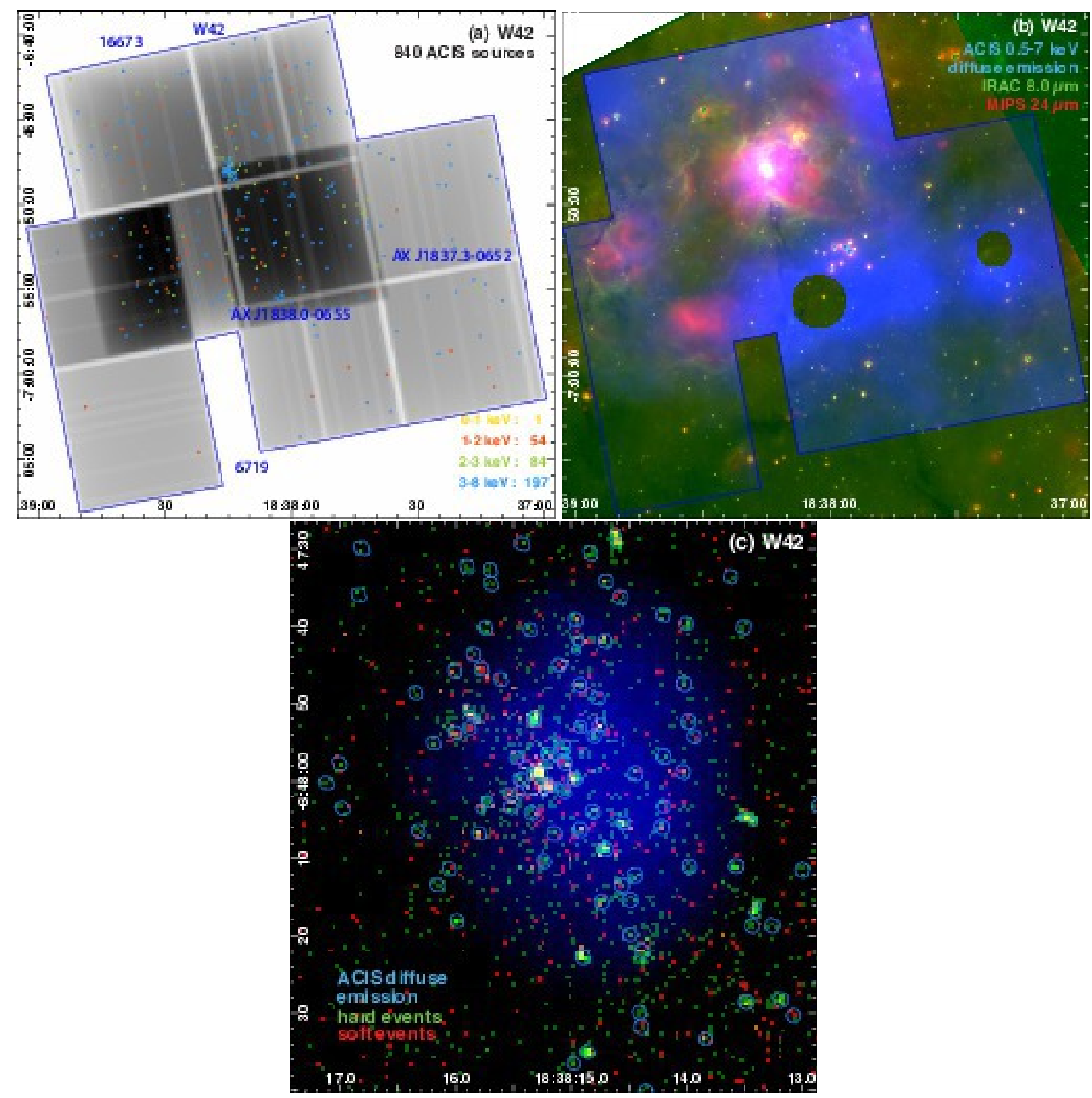

Figure 11. W42 and RSGC1. (a) ACIS exposure map with brighter ( $\geq 5$ net counts) ACIS point sources overlaid; colors denote median energy for each source. ObsID numbers and regions named in the text are shown in blue. (b) ACIS diffuse emission in the Spitzer context. The two pulsars in the field, AX J1838.0-0655 and AX J1837.3-0652 (Gotthelf \& Halpern 2008), along with the brightest parts of their associated diffuse X-ray emission, have been masked so that fainter diffuse X-ray emission across the field can be displayed. (c) ACIS event data and diffuse emission at the center of W42.

2008). Despite almost 74 ks of ACIS-I integration, we still do not detect any of the red supergiants 
in RSGC1 (Table 1 in Figer et al. 2006). There are many X-ray sources in this region of the mosaic, however, and several are quite hard. These highly-absorbed sources may well be members of RSGC1.

Gotthelf \& Halpern (2008) analyzed ObsID 6719 and noted that their soft X-ray source \#12 is the high-proper-motion spectroscopic binary HD 171999. Our source c747 (CXOU J183758.77-064822.2) is their source \#12. Nearby in our data is the similarly soft X-ray source c738 (CXOU J183758.67064826.0). ObsID 16673 was obtained 9.5 years after ObsID 6719. Using SIMBAD proper motions for HD 171999, we calculate that this source should have moved about $-1.2^{\prime \prime}$ in RA, $-3.8^{\prime \prime}$ in Dec, in the 9.5 years between ACIS observations. Separations between c747 and c738 are about $-1.5^{\prime \prime}$ in RA, $-3.8^{\prime \prime}$ in Dec. Thus we conclude that our sources c747 and c738 are in fact the same X-ray source, the counterpart to HD 171999.

Diffuse X-ray emission around the pulsars AX J1838.0-0655 and AX J1837.3-0652 (Gotthelf \& Halpern 2008) extends far beyond the brightest regions that we masked and dominates our ACIS mosaic of this field (Figure 11b). The bright PWN around pulsar AX J1838.0-0655 (our piled source c989) may have been over-reconstructed into point sources by our machinery, thus the six sources surrounding c989 may be spurious.

Fainter X-ray emission pervades much of the wider field, filling bubbles and voids in the Spitzer images. The brightest unresolved emission at the center of the W42 cluster (Figure 11c) undoubtedly includes a substantial contribution from faint cluster members; spectral analysis will be required to establish if any of this emission is truly diffuse.

\subsection{W33 and $\mathrm{Cl} 1813-178$}

The other targets in our recent GO program to study MSFRs at a range of ages were the very young W33 complex and the older, more distant YMC Cl 1813-178. As we found in the ACIS mosaic of W42 and RSGC1, here again we have a superposition of unrelated MSFRs separated by just a few arcminutes on the sky (Figure 12). In this case, $\mathrm{Cl}$ 1813-178 is so big and so well-populated with X-ray sources that it will be a challenge to assign membership in the part of the field where it overlaps with the foreground W33 complex.

The W33 MSFR consists of a chain of very young stellar clumps and sparse clusters. It appears that these clusters formed separately and nearly simultaneously (Beck et al. 1998); this may indicate that they formed at sausage-instability density enhancements along a massive molecular filament (Jackson et al. 2010; Chandrasekhar \& Fermi 1953). The distance to W33 has recently been firmly established at $2.4 \mathrm{kpc}$ via maser trigonometric parallax (Immer et al. 2013).

The ACIS-I aimpoint for our study of W33 was centered on W33 Main (Figure 13), which contains at least 5 mid-O stars (Beck et al. 1998) ionizing at least 3 distinct UCHıRs, separated by dense material. We find a clear concentration of X-ray sources consistent with the cluster position as given by Immer et al. (2014) (Figure 13(b)), but no counterparts to the infrared sources IRS1, IRS2, or IRS3 from Dyck \& Simon (1977) (using SIMBAD positions).

W33A, a deeply-embedded MSFR that features a well-studied MYSO (Davies et al. 2010), is captured at the northeast corner of the ACIS-I field. W33A may be forming due to the intersection of two massive molecular filaments (Galván-Madrid et al. 2010). We find five faint X-ray sources in a clump at the location of W33A; all have median energies above $3 \mathrm{keV}$, indicating that they are highly obscured. If these sources are associated with star formation in W33A, they indicate either that X-ray emission turns on very early in the star formation process or that W33A has experienced 


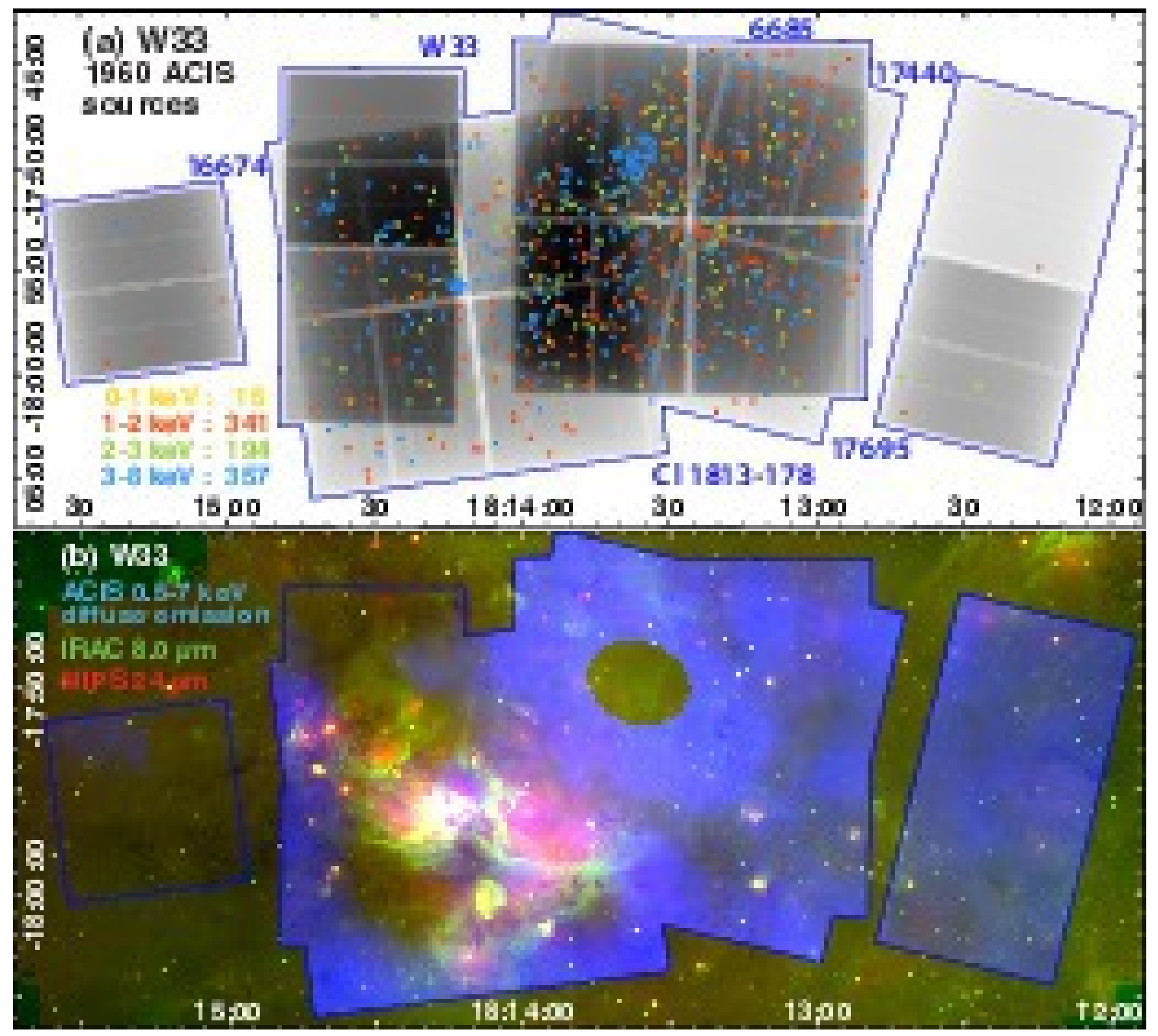

Figure 12. W33. (a) ACIS exposure map with brighter ( $\geq 5$ net counts) ACIS point sources overlaid; colors denote median energy for each source. ObsID numbers and regions named in the text are shown in blue. (b) ACIS diffuse emission in the Spitzer context. The bright SNR G12.82-0.02 has been masked.

multiple epochs of star formation, with today's MYSO and dusty cores representing current and future star formation while the X-ray sources represent an older population.

The ACIS data cover four more clusters associated with Hir regions across the W33 complex: radio region W33B and IR clusters cl1, cl2, and Mercer 1 (Messineo et al. 2011). While all of these regions contain X-ray sources, none of them shows a clear central concentration indicative of an X-ray clump, as we saw in W33 Main and W33A.

The monolithic YMC Cl 1813-178 was discovered serendipitously (Messineo et al. 2008) just 13' west of W33 Main, but Messineo et al. (2011) estimate that it is much more distant (3.8-4.8 kpc), 

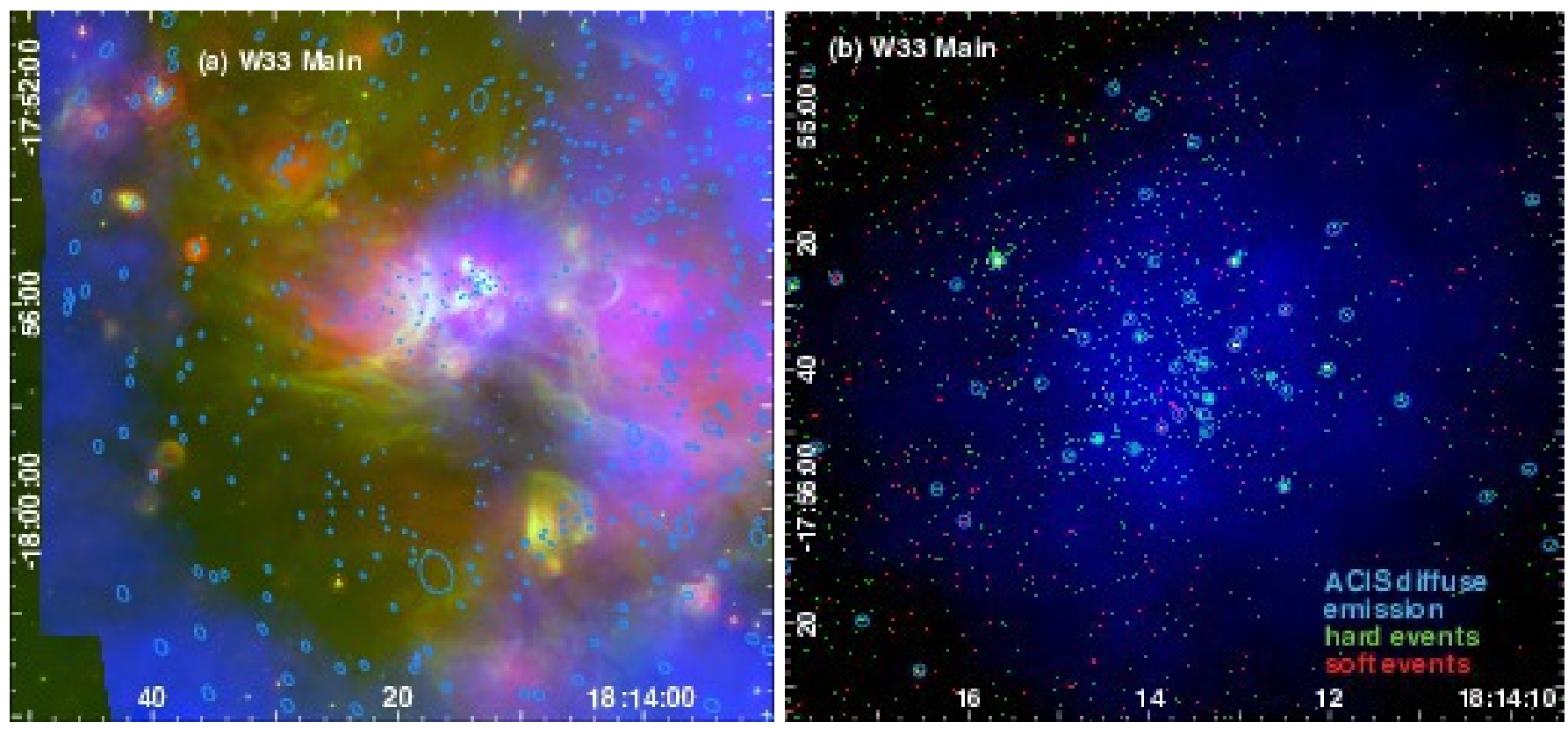

Figure 13. W33 Main. (a) Zoomed version of Figure 12(b) for W33 Main, with point source extraction regions. (b) ACIS event data and diffuse emission on W33 Main.

hence unrelated to W33. Cl 1813-178 is one of fewer than 20 YMCs known in the Milky Way (Clark et al. 2013), with a mass of $10^{4} \mathrm{M}_{\odot}$ and many known massive stars (Messineo et al. 2011).

Helfand et al. (2007) analyzed the original 30-ks ACIS-I observation of this field (which targeted SNR G12.82-0.02) and catalogued 75 X-ray sources; we recover all of those sources in our analysis. Our combined (ACIS GTO + archival) 59-ks Chandra dataset provides a wealth of candidate members of $\mathrm{Cl}$ 1813-178 (Figure 14), with 572 X-ray sources within the reported cluster radius of 3.5' (Messineo et al. 2011) and hundreds more beyond that.

We have X-ray counterparts to at least 16 of the 25 massive stars listed in Messineo et al. (2011, Table 1). They find two Wolf-Rayet (WR) stars in this cluster: their \#4 and \#7, both of type WN7. The first of these, \#4, is our source c807 (CXOU J181314.20-175343.4), with 348 net counts and a median energy of $3.6 \mathrm{keV}$; a spectral fit gives $N_{H}=8 \times 10^{22} \mathrm{~cm}^{-2}, k T=2.8 \mathrm{keV}$, and $L_{X}=1.2 \times 10^{33} \mathrm{erg} \mathrm{s}^{-1}$. The second of these, \#7, is our source c1281 (CXOU J181322.48-175350.2), with 68 net counts and a median energy of $2.8 \mathrm{keV}$. It is crowded with the faint source c1286 (CXOU J181322.56-175350.1), with 6 net counts and median energy $3.7 \mathrm{keV}$. A spectral fit to c1281 gives $N_{H}=4 \times 10^{22} \mathrm{~cm}^{-2}, k T=2.6 \mathrm{keV}$, and $L_{X}=1.3 \times 10^{32} \mathrm{erg} \mathrm{s}^{-1}$. Based on the high thermal plasma temperatures and X-ray luminosities for these WR stars, we concur with Messineo et al. that they are likely CWBs.

We also fit the X-ray spectrum of Messineo et al. (2011) source \#5, which they find to be a late-O star but with an unusually hard X-ray spectrum. This is our source c1346 (CXOU J181323.71175040.5), with 265 net counts and a median energy of $3.0 \mathrm{keV}$; fit parameters are $N_{H}=2.0 \times$ $10^{22} \mathrm{~cm}^{-2}, k T>10 \mathrm{keV}$, and $L_{X}=3.5 \times 10^{32} \mathrm{erg} \mathrm{s}^{-1}$. This spectrum is equally well fit by a power law $\left(\right.$ TBabs ${ }^{*}$ pow in XSPEC) with $N_{H}=2.0 \times 10^{22} \mathrm{~cm}^{-2}$, slope $\Gamma=1.5$, and $L_{X}=3.6 \times 10^{32} \mathrm{erg} \mathrm{s}^{-1}$. As Messineo et al. suggest, this source may be another CWB. Its spectrum is so hard that it might be compared to that found for source I20S in IRAS 20126+4104 (Anderson et al. 2011). 

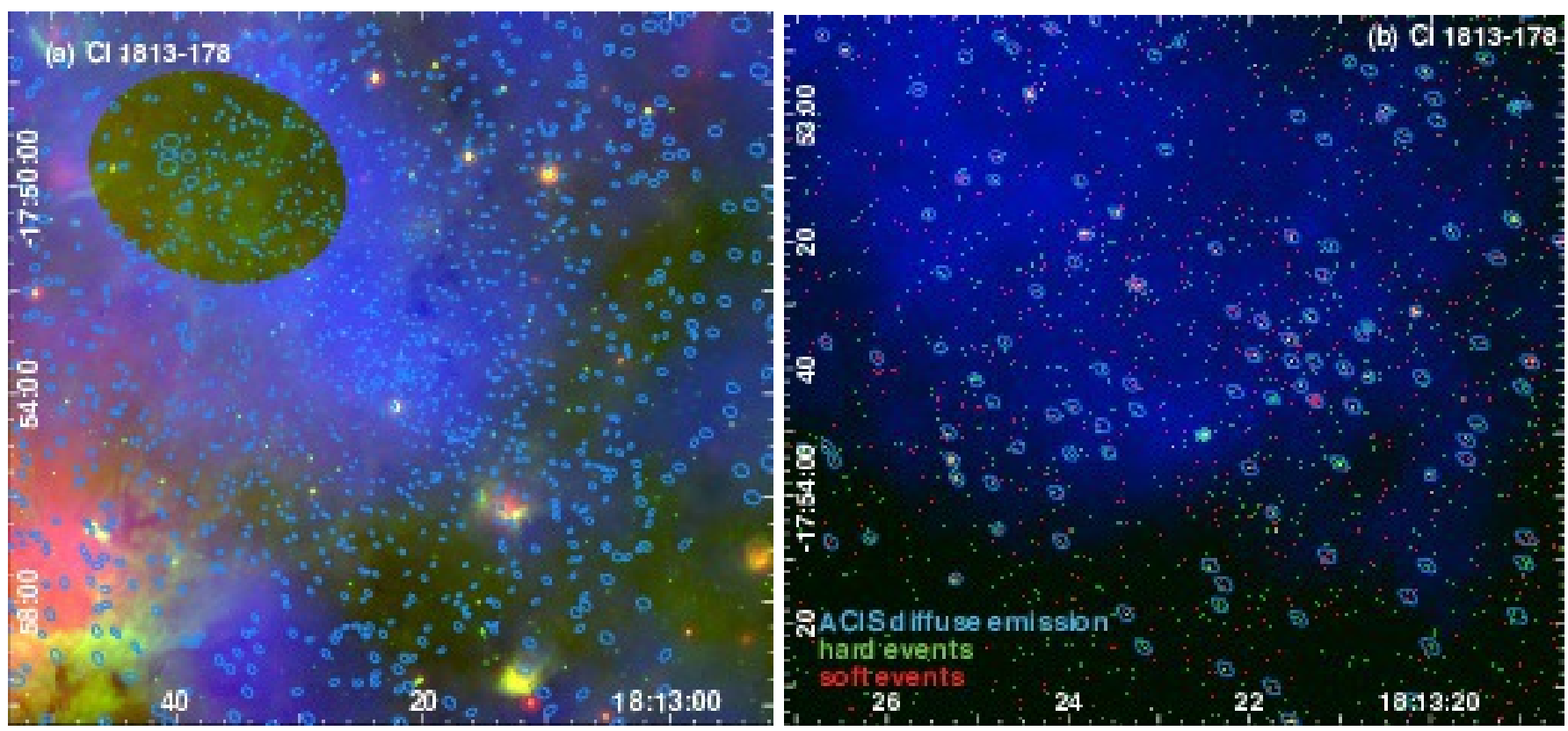

Figure 14. Cl 1813-178. (a) Zoomed version of Figure 12(b) for $\mathrm{Cl} 1813-178$, with point source extraction regions. (b) ACIS event data and diffuse emission for the center of $\mathrm{Cl}$ 1813-178.

Our ACIS mosaic reaches approximately the same sensitivity in both W33 and Cl 1813-178; W33 is closer but more obscured. As shown in all figures in this section, diffuse X-ray emission pervades the entire W33 mosaic. As we saw in our W42 mosaic, the brightest diffuse emission here is associated with the PWN around a pulsar (PSR J1813-1749) and its SNR (G12.82-0.02) (Funk et al. 2007; Helfand et al. 2007; Gotthelf \& Halpern 2009). Some of our catalogued point sources that are superposed on this bright diffuse emission could be artifacts of image reconstruction; identifying counterparts to these X-ray sources at longer wavelengths is the best way to set aside such a concern. Fainter diffuse emission extends to the west across the entire $\mathrm{Cl}$ 1813-178 ACIS pointing. It appears at the W33 Main and Cl 1813-178 cluster centers and around the edges of the W33 ACIS-I field. It is faint but present on the easternmost ACIS-S CCD. The W33 MSFR probably shadows background diffuse emission across most of the W33 pointing.

\section{9. $N G C 7538$}

NGC 7538 is a clumpy Outer Galaxy MSFR, made up of several separate star-forming sites with a range of ages (McCaughrean et al. 1991; Balog et al. 2004; Ojha et al. 2004), similar to W33. It hosts the O3V star IRS6 and the O9V star IRS5 (Puga et al. 2010), along with a variety of MYSOs that are well-studied in the radio (e.g., Beuther et al. 2017).

The Chandra data were analyzed recently as part of two multiwavelength studies (Mallick et al. 2014; Sharma et al. 2017), finding 182 and 190 X-ray point sources respectively, but neither of these papers gives full catalogs for their Chandra sources. Mallick et al. tabulate 27 X-ray sources with NIR counterparts in the IRS1-3 and IRS9 regions; they provide some X-ray properties for that subset of their X-ray detections. We recover all of these sources in our analysis and go on to find almost 500 X-ray sources and diffuse X-ray emission in this single 30-ks ACIS-I observation (Figures 15(a) and $(b))$. 


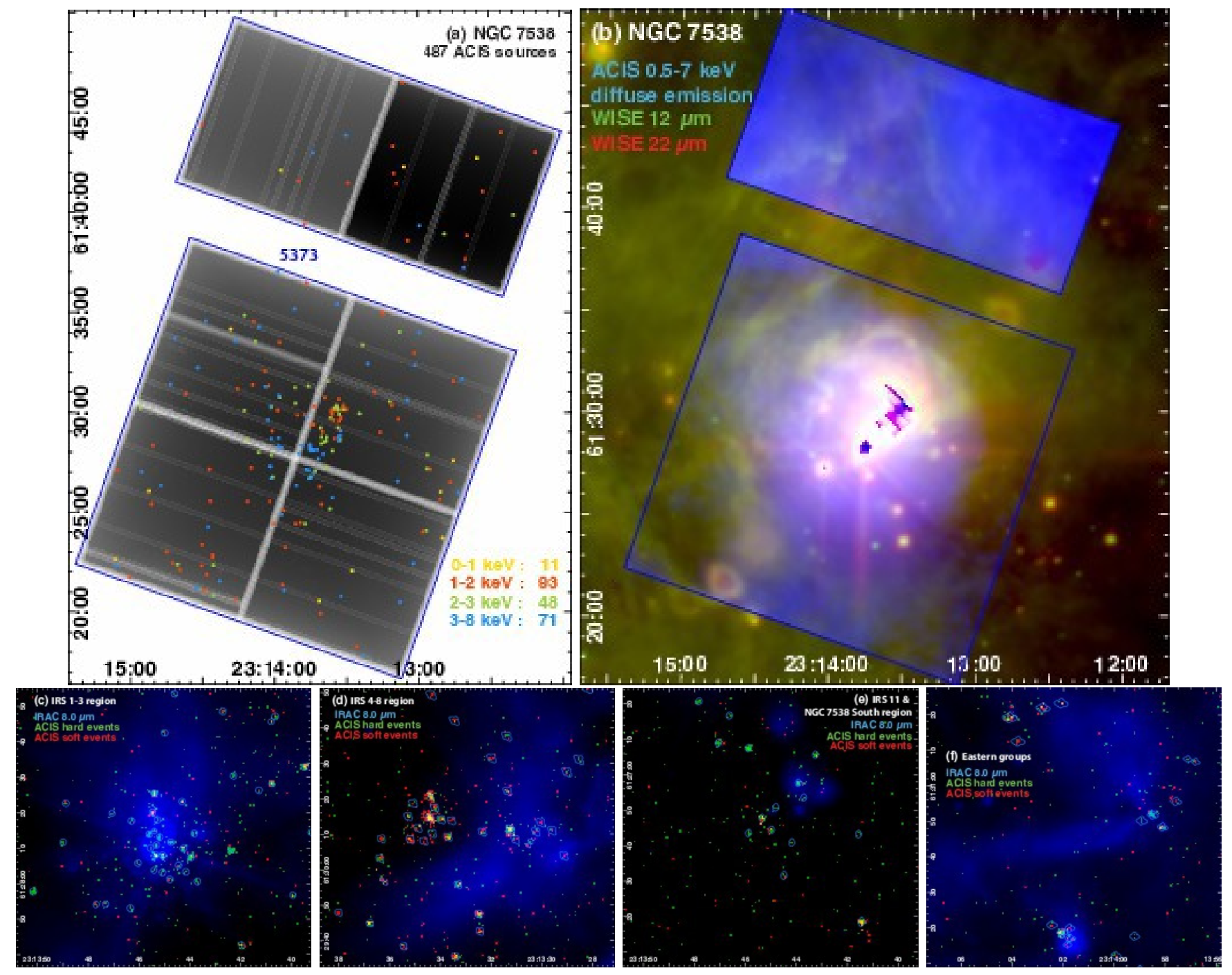

Figure 15. NGC 7538. (a) ACIS exposure map with brighter ( $\geq 5$ net counts) ACIS point sources overlaid; colors denote median energy for each source. The ObsID number is shown in blue. (b) ACIS diffuse emission in the WISE context. Spitzer data have incomplete coverage of this area. (c)-(f) ACIS event images for select star-forming clumps, with Spitzer IRAC $8 \mu$ m images included for orientation. (c) The IRS1-3 region. Saturated sources dominate the center of the IRAC image. (d) The IRS4-8 region. (e) The IRS11 and NGC 7538 South region. (f) The globules noted by Sharma et al. (2017).

In fact we find clumps of X-ray sources associated with all the well-known star-forming regions in NGC 7538: IRS1-3, IRS4-8, IRS9, IRS11/NGC 7538S, and the globules described by Sharma et al. (2017). ACIS images of most of these regions are presented in Figures 15(c)-(f); the IRS9 region is omitted because it has few X-ray sources and the IRAC $8 \mu \mathrm{m}$ image is dominated by the single bright source IRS9 itself. Sharma et al. (2017) give short summaries of these star-forming regions; they all harbor massive stars or MYSOs, many ionizing various types of His regions. Here we briefly describe X-ray counterparts to the famous IRS sources.

IRS1-3 (Figure 15(c)). We find many X-ray sources in this young MSFR. Chandra source c343 (CXOU J231345.32+612810.9) is the counterpart to the MYSO IRS1; it has just 5 X-ray events but all have energies $>3 \mathrm{keV}$, with a median energy of $4.4 \mathrm{keV}$. This indicates that the X-ray source is highly 
obscured, as expected for this massive protostar. Chandra source c351 (CXOU J231345.47+612820.1) is the counterpart to IRS2, an $09.5 \mathrm{~V}$ star; its 8 counts have a median energy of $1.6 \mathrm{keV}$. IRS3 has no X-ray counterpart in our data.

IRS4-8 (Figure 15(d)). This is the main star-forming complex in NGC 7538; it is well-populated with X-ray sources. Chandra source cc228 (CXOU J231330.22+613010.7) is the counterpart to the O9V star IRS5, with 9 counts and a median energy of $1.4 \mathrm{keV}$, similar to IRS2. Chandra source c222 (CXOU J231334.40+613014.6) is the counterpart to the O3V star IRS6, with 400 counts and a median energy of $1.4 \mathrm{keV}$. A spectral fit yields $N_{H}=1.7 \times 10^{22} \mathrm{~cm}^{-2}, k T=0.8 \mathrm{keV}$, and $L_{X}=8 \times 10^{32} \mathrm{erg} \mathrm{s}^{-1}$. Sources IRS4, IRS7, and IRS8 have no X-ray counterparts.

IRS9. The faint Chandra source c460 (CXOU J231401.78+612718.8) is the counterpart to the MYSO IRS9. It has just 8 counts and an extreme median energy of $5.0 \mathrm{keV}$; event energies span the wide range of $1.5-7.5 \mathrm{keV}$. Sandell et al. (2005) find multiple outflows in this region, suggesting an embedded cluster rather than a single MYSO, but we find just a few X-ray sources here.

IRS11/NGC 7538S (Figure 15(e)). Chandra source c322 (CXOU J231343.92+612657.6) may be a match to the MYSO IRS11. It has 17 counts and a median energy of $4.0 \mathrm{keV}$, with event energies ranging over 2.6-5.0 keV. The spectral fit is not well-constrained, but the source is certainly highly obscured, with $N_{H}>20 \times 10^{22} \mathrm{~cm}^{-2}, k T \sim 1 \mathrm{keV}$ plasma with $L_{X} \sim 1 \times 10^{33} \mathrm{erg} \mathrm{s}^{-1}$. We do not detect an X-ray counterpart to NGC 7538 S.

Globules (Sharma et al. 2017) (Figure 15(f)). These host small groups of X-ray sources at the eastern edge of NGC 7538. Sharma et al. noted that they resemble bright-rimmed clouds and point back to the O3 star IRS6.

The brightest diffuse X-ray emission in the ACIS observation is far off-axis on the ACIS-S chips. On-axis, a diffuse enhancement is coincident with the central cavity around the cluster. Faint patches of diffuse X-rays extend north and south to the edges of the ACIS-I field, anticoincident with the WISE mid-IR emission. Luisi et al. (2016) find ionizing radiation leaking past the primary photodissociation region (PDR) to a second PDR northeast of the main bubble, demonstrating the influence of MSFRs on the surrounding ISM. Diffuse X-ray emission extends at least as far as the second PDR to the northeast. It also spreads to the south and southwest, past the main NGC 7538 bubble seen in the WISE data, once again demonstrating that the hot ISM around MSFRs is more complex and extensive than might be predicted from IR data alone.

\subsection{0. $\quad$ G333}

The elongated G333 GMC hosts several obscured MSFRs, spread over 80 pc across its long axis (e.g., Russeil et al. 2005; Nguyên et al. 2015). G333 has formed several massive young clumps and clusters, all at nearly the same time and with nearly regular spacing along the cloud. These clusters are too young to have hosted supernovae and their wind-blown bubbles do not overlap significantly, so it is unlikely that we are witnessing a "sequential triggering" process (Elmegreen \& Lada 1977).

G333 may be a more evolved version of the 80-pc-long massive molecular filament and IRDC "Nessie" (Jackson et al. 2010). Near-IR dust extinction maps (Kainulainen et al. 2011) show that IRDCs are often surrounded by molecular clouds with $>10$ times more mass; thus a massive (but not extraordinary) IRDC of $10^{4} \mathrm{M}_{\odot}$ can easily represent just the densest part of a typical $10^{5} \mathrm{M}_{\odot}$ GMC. So G333 may be a rare example of a very massive IRDC with an unusually favorable sky orientation that has nearly completely collapsed into regularly-spaced sites of coeval massive star formation. Soon supernovae in these embedded clusters will destroy the last vestiges of the original IRDC and 
start blowing superbubbles to feed the hot ISM, as we see in Carina (Townsley et al. 2011a,b) and NGC 6357 (MOXC1). But since the underlying elongated structure still remains in G333, we can study the transformation of a massive molecular filament into a cluster of clusters. Similar to G352 described above but more distant (so the entire GMC can be captured in a few ACIS-I pointings), G333 provides an important observational and evolutionary link between giant molecular filaments such as Nessie and cluster-of-clusters star-forming complexes such as Carina.
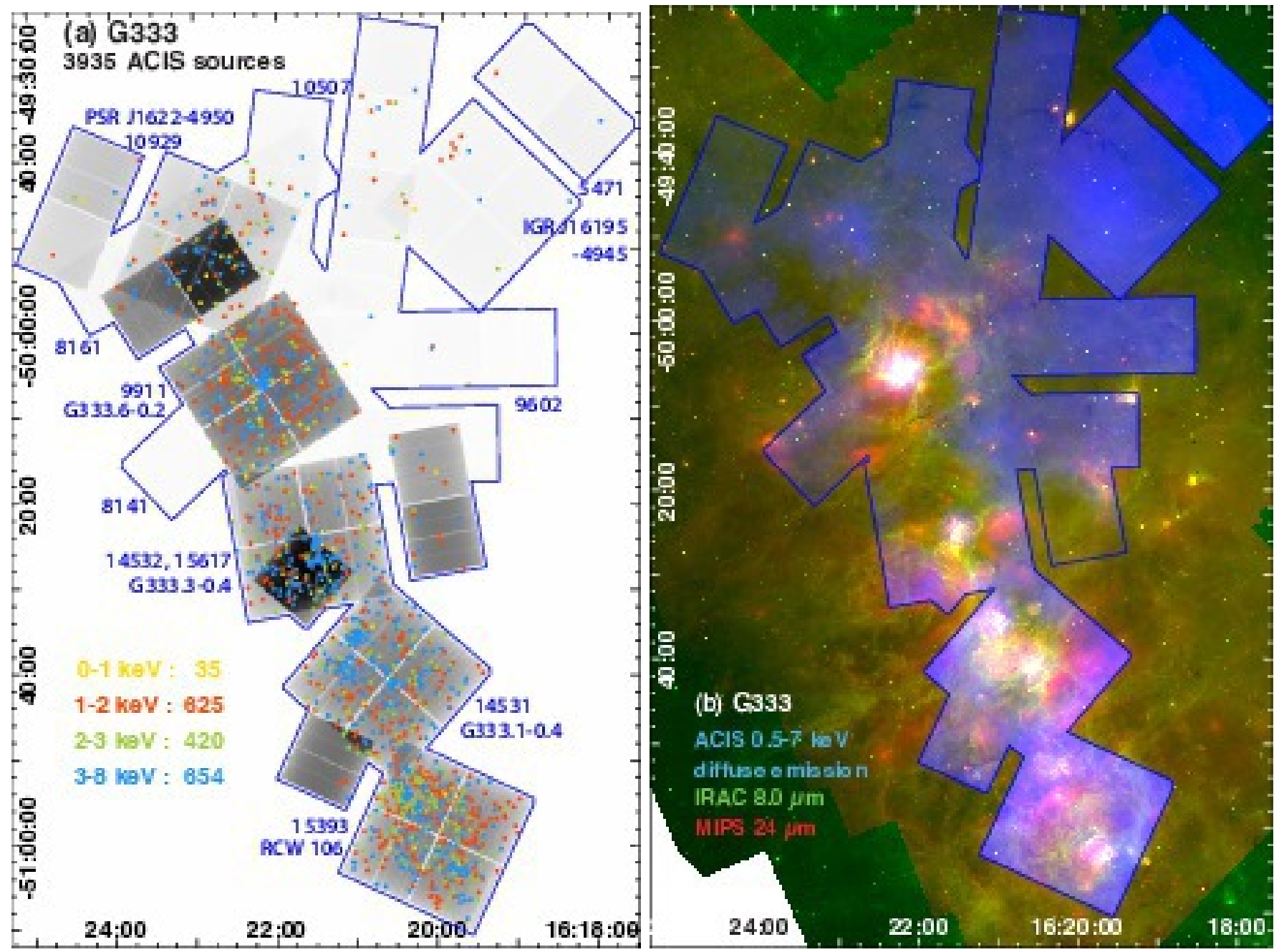

Figure 16. G333. (a) ACIS exposure map with brighter ( $\geq 5$ net counts) ACIS point sources overlaid; colors denote median energy for each source. ObsID numbers and regions named in the text are shown in blue. The image appears white for regions with $<5$ ks exposure. (b) ACIS diffuse emission in the Spitzer context.

This study required four 60-ks ACIS-I pointings to mosaic MSFRs across the entire G333 GMC. We included additional archival Chandra data on surrounding pointings to create a wide-field, highresolution X-ray mosaic of the entire G333 GMC (Figure 16). The young SNR RCW 103 lies just off the southwest edge of our G333 mosaic. We have omitted its ACIS data from our mosaic because there is a recent analysis of those data (Frank et al. 2015). It is also extremely bright and swamps the fainter diffuse X-ray emission in our mosaic. As in MOXC1, we continue to assume a distance 
of 2.6 kpc to G333 (Figuerêdo et al. 2005; Roman-Lopes et al. 2009). Recent papers (e.g., Lo et al. 2015; Nguyên et al. 2015) use a distance of 3.6 kpc to this GMC; assuming this larger distance would increase our X-ray source luminosities by nearly a factor of two. Below we describe each of our four main ACIS-I pointings on G333 MSFRs.

\subsubsection{G333.6-0.2}

The most prominent MSFR in G333 is the bipolar bubble and giant Hit region G333.6-0.2 (Figure $17(\mathrm{a}))$. We presented a single-ObsID (60.1 ks) ACIS-I study of this region in MOXC1. The MOXC2 analysis used slightly more data $(61.6 \mathrm{ks})$. In both epochs of analysis ACIS reveals over 100 $\mathrm{X}$-ray sources in a central compact cluster powering the giant His region, a distributed population of over 500 more point sources across this ACIS-I pointing, and soft diffuse X-ray emission tracing hot plasma apparently leaking from the embedded cluster (Figure 17(b)). As we found for the main ridge in NGC 6334, MOXC1 and MOXC2 catalogs are the same for brighter sources but find different sources at the faint limit. As before, we favor the MOXC2 results because they reflect improvements in our analysis methodologies over the years.
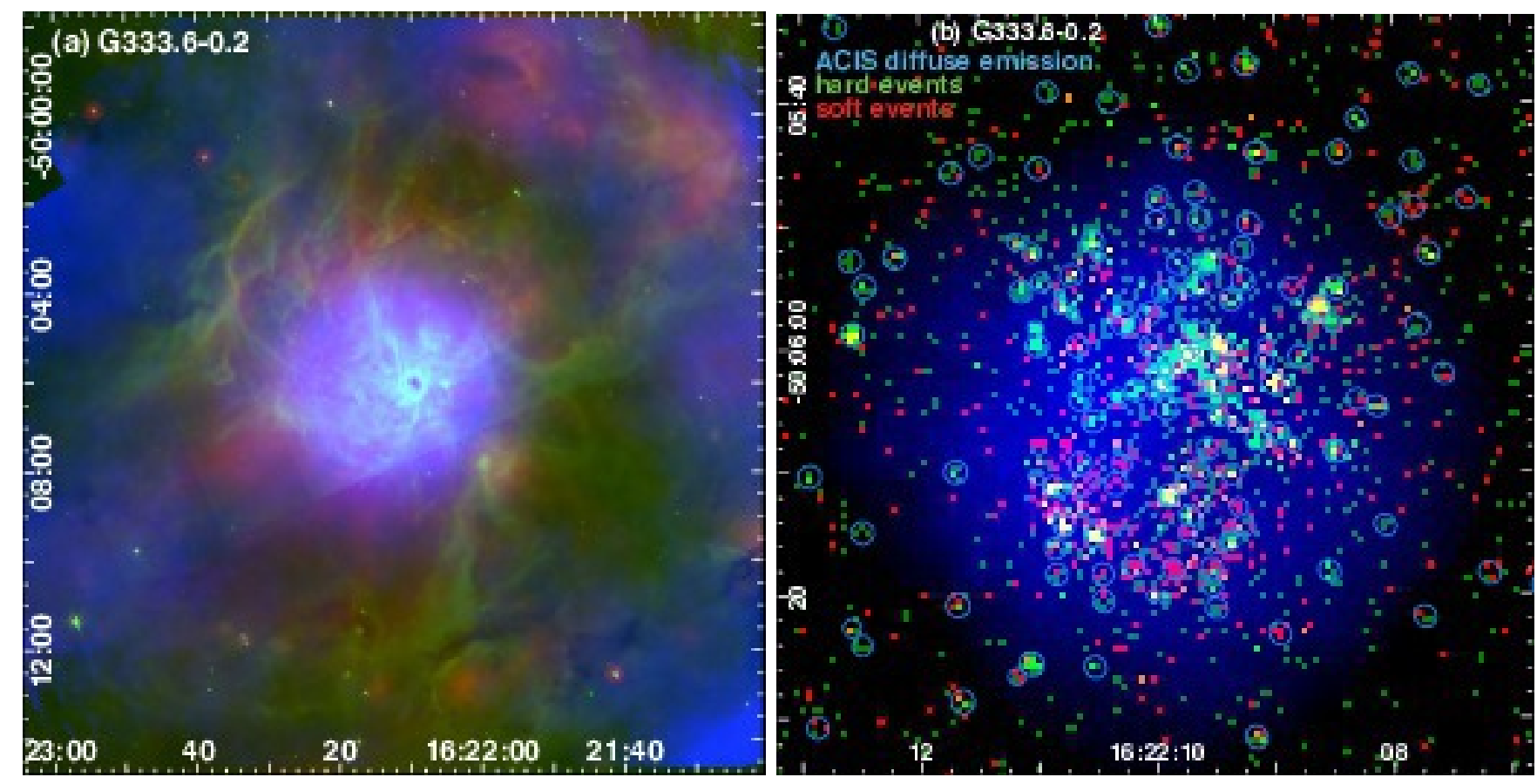

Figure 17. G333.6-0.2. (a) Zoomed version of Figure 16(b) for G333.6-0.2. (b) MOXC2 ACIS event data and diffuse emission for the center of G333.6-0.2.

\subsubsection{G333.3-0.4}

This pointing (Figure 18) contrasts an IR-bright MSFR with the only part of the G333 GMC that is not bright at $24 \mu \mathrm{m}$. The upper half of Figure 18(a) shows this dark bay; we see no diffuse X-ray emission from this region. Water masers are found even in the IR-dark part of the field, indicating ongoing massive star formation (Breen et al. 2007). A near-IR study of G333.3-0.4 (Roman-Lopes et al. 2003) found 41 reddened massive stars in a $1^{\prime}$ cluster behind $\left\langle A_{V}\right\rangle \sim 28$ mag, with spectral types 
$\sim$ O5.5-B5. We find X-ray counterparts to at least 13 of those massive stars, sometimes resolving two or three X-ray sources at the location of the IR source.
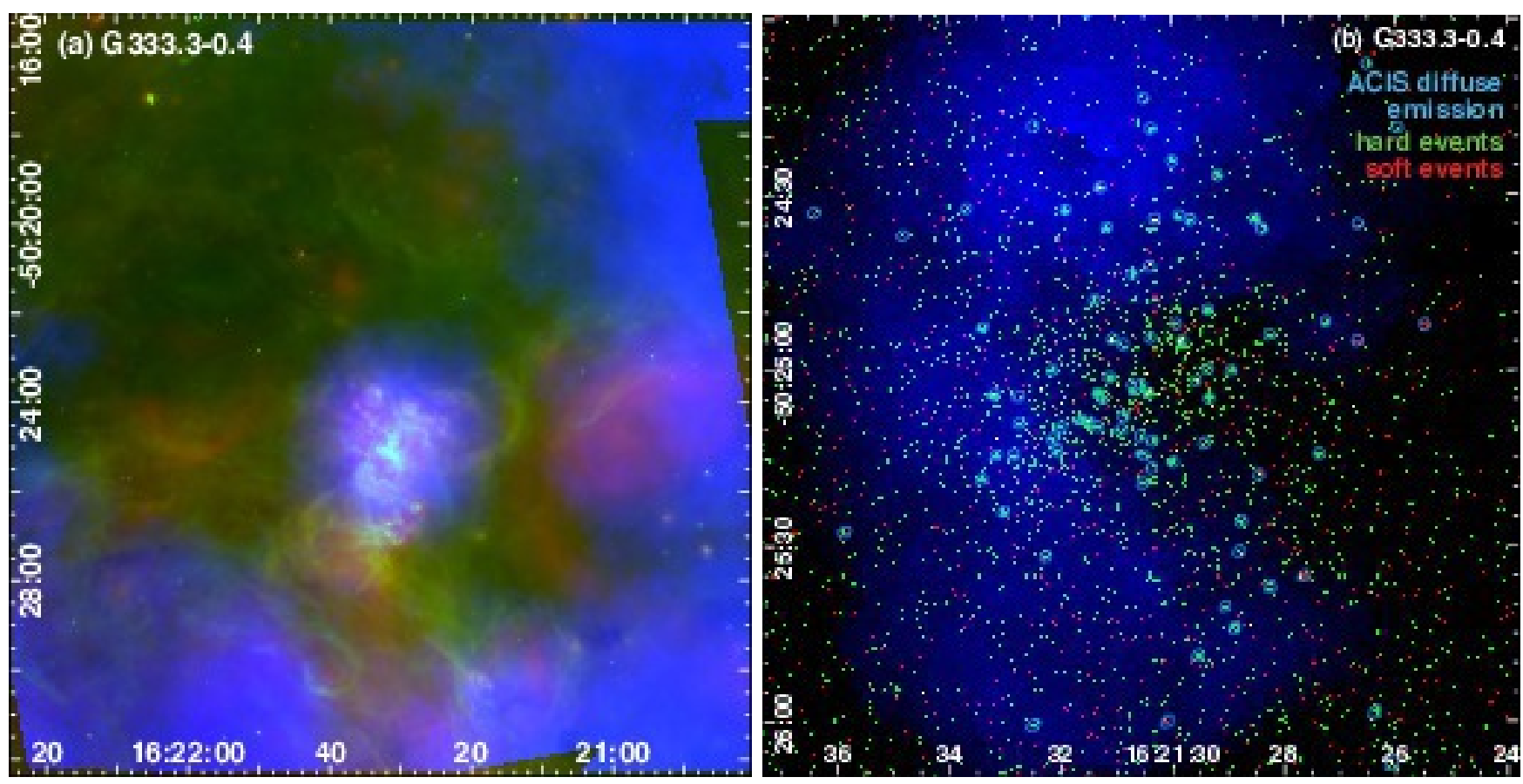

Figure 18. (a) Zoomed version of Figure 16(b) for G333.3-0.4. (b) ACIS event data and diffuse emission for the center of G333.3-0.4.

The MSFR G333.3-0.4 is associated with IRAS 16177-5018, powered by an O3If ${ }^{+}$star at $2.6 \pm$ $0.7 \mathrm{kpc}$ (Roman-Lopes et al. 2009). Our X-ray counterpart to that star is c5623 (CXOU J162131.60502508.3 ), with 13 net counts and a median energy of $3.2 \mathrm{keV}$; it has two close neighbors (each $<1.2^{\prime \prime}$ away). With so few counts, spectral fit parameters for c5623 are not well-constrained, but they are roughly $N_{H} \sim 13 \times 10^{22} \mathrm{~cm}^{-2}, k T \sim 0.9 \mathrm{keV}$, and $L_{X} \sim 4 \times 10^{32} \mathrm{erg} \mathrm{s}^{-1}$.

In Figure 18(a) we do see diffuse emission from the G333.3-0.4 cluster and around the edges of this field, sometimes filling Spitzer bubbles. The center of the cluster (Figure 18(b)) contains a substantial number of X-ray sources; the overdensity of hard events not captured by detected sources implies that there are many more X-ray sources just below the detection limit. Diffuse X-ray emission is seen around the cluster but is fainter in the center, perhaps shadowed by the high absorbing column in this region. The X-ray sources are typically hard and faint, again probably due to high absorption. No other major grouping of X-ray sources is found in this pointing.

\subsubsection{G333.1-0.4}

This pointing (Figure 19) captures two powerful His regions, G333.12-0.45 and G333.03-0.45 (GAL 333.1-00.4 and GAL 333.0-00.4 in SIMBAD). A near-IR study of G333.12-0.45 also finds a cluster distance of $2.6 \mathrm{kpc}$, a range of extinctions $\left(A_{V} \sim 12-32 \mathrm{mag}\right)$, several MYSOs with spectral types $\mathrm{O} 4-\mathrm{B} 4$, and a total cluster mass of $>10^{3} \mathrm{M}_{\odot}$ (Figuerêdo et al. 2005), but no source positions are provided. G333.12-0.45 shows direct evidence for large-scale infall (Lo et al. 2015), implying that it is very young. 


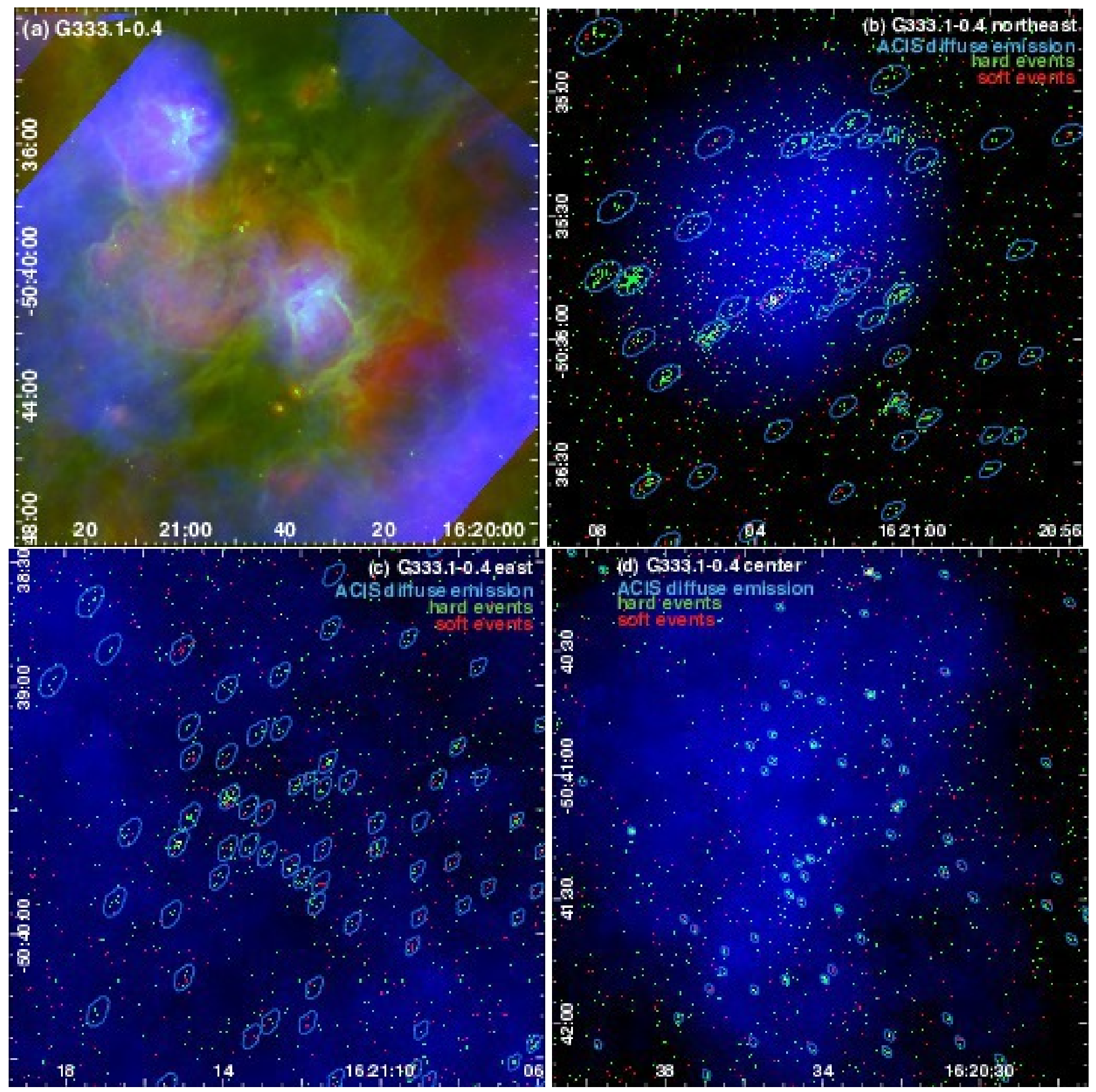

Figure 19. (a) Zoomed version of Figure 16(b) for G333.1-0.4. (b)-(d) ACIS event data and diffuse emission for source concentrations in G333.1-0.4. (b) G333.1-0.4 northeast, with the brightest diffuse emission in the G333.1-0.4 pointing. (c) G333.1-0.4 east. (d) G333.1-0.4 center.

The grouping of X-ray sources closest to G333.12-0.45 is shown in Figure 19(b), in the northeast region of this pointing. Bright diffuse X-ray emission is seen here. This region is several arcminutes off-axis where ACIS PSFs are large and sensitivity is reduced, thus point sources are typically faint; the diffuse emission may be due partially to unresolved pre-MS stars from this obscured cluster. 
Another group of X-ray sources is shown in Figure 19(c), in the eastern part of this ACIS pointing. Faint diffuse X-ray emission pervades the field. There is an UCHıR candidate here (Mookerjea et al. 2004). These X-ray sources may be part of GLIMPSE IR cluster \#78 (Mercer et al. 2005).

The Hir region G333.03-0.45 is not as well-studied as G333.12-0.45 but shows a large number of dust cores and UCHirR candidates (Mookerjea et al. 2004). It is as bright in Lyman continuum as the other G333 MSFRs (Conti \& Crowther 2004). G333.03-0.45 is at the center of this ACIS pointing. It shows diffuse X-ray emission distributed throughout a fairly loose grouping of point sources (Figure 19(d)).

\subsection{4. $R C W 106$}

The final pointing (Figure 20) in our G333 mosaic of MSFRs again shows diffuse X-ray emission at the periphery and in a Spitzer bubble, with strong shadowing at the center (Figure 20(a)). We find two main clumps of X-ray sources. The first is on the northeast side of the pointing (Figure 20(b)). Two bright X-ray sources are found here: c2691 (CXOU J162026.75-505417.1; 398 net counts, median energy $1.7 \mathrm{keV}$ ) and c2412 (CXOU J162022.75-505420.4; 110 net counts, median energy $4.2 \mathrm{keV}$, variable). A spectral fit to c2691 requires two thermal plasma components, with $N_{H}=2.2 \times 10^{22} \mathrm{~cm}^{-2}$, $k T 1=0.5 \mathrm{keV}, k T 2>3 \mathrm{keV}$, and $L_{X}=9.0 \times 10^{32} \mathrm{erg} \mathrm{s}^{-1}$. The harder source, c2412, can be fit with a one-temperature thermal plasma, although that temperature is so high that it is not well-constrained by the ACIS data: $N_{H}=10 \times 10^{22} \mathrm{~cm}^{-2}, k T>10 \mathrm{keV}$, and $L_{X}=1 \times 10^{32} \mathrm{erg} \mathrm{s}^{-1}$.

The second clump of X-ray sources is on the southwest side of the pointing, in a prominent Spitzer bubble (Figure 20(c)). These sources may be associated with GLIMPSE IR cluster \#76 (Mercer et al. 2005). 


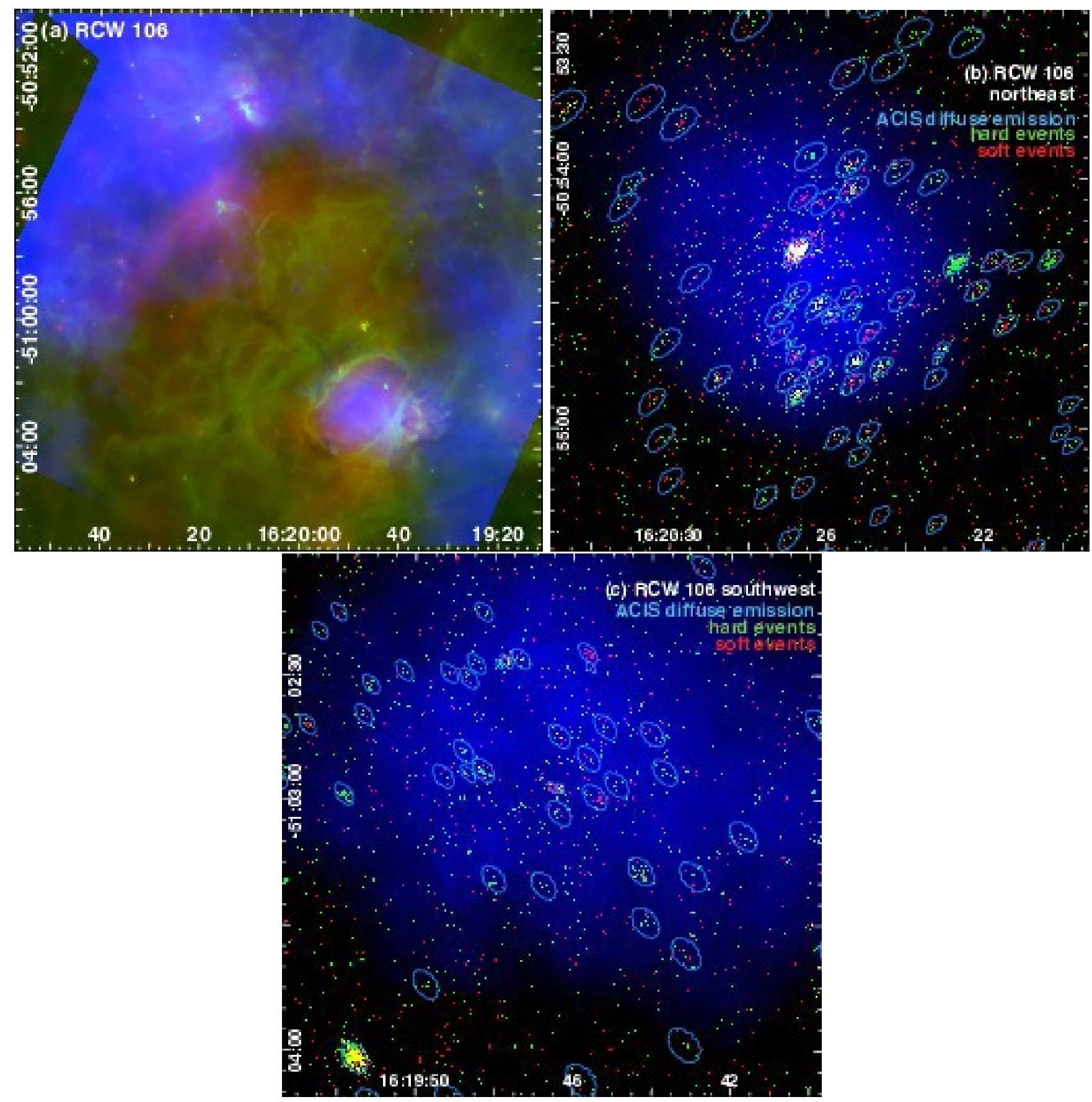

Figure 20. (a) Zoomed version of Figure 16(b) for RCW 106. (b)-(c) ACIS event data and diffuse emission for source concentrations in RCW 106. (b) RCW 106 northeast. (c) RCW 106 southwest. 


\subsubsection{Archival Pointings}

We included several archival Chandra datasets in the G333 mosaic, to broaden the field and provide a wider context for the diffuse X-ray emission. ObsIDs 8141, 8161, 9602, and 10507 were from the ChIcAGO project (Anderson et al. 2014). ObsID 10929 re-observed a bright ChIcAGO source, which was determined to be the magnetar PSR J1622-4950 (Levin et al. 2010; Anderson et al. 2012). This is our source c8972 (CXOU J162244.91-495052.8); it is piled up in ObsID 8161. We find prominent diffuse X-ray emission surrounding the magnetar (Figure 21(a)). This could be the X-ray signature of its SNR; Anderson et al. (2012) found a radio arc just north of this region (G333.9+0.0) and suggested that it might be a SNR associated with PSR J1622-4950. Our diffuse X-ray emission could be tracing hot plasma filling that radio-bright partial shell. Additionally, Anderson et al. showed PSR J1622-4950 to be coincident with a potentially nonthermal diffuse radio source ("Source A") that may be a PWN associated with the magnetar. Since the diffuse X-ray emission is concentrated in the vicinity of PSR J1622-4950 and the diffuse radio source, this adds further support for the existence of a magnetar wind nebula.
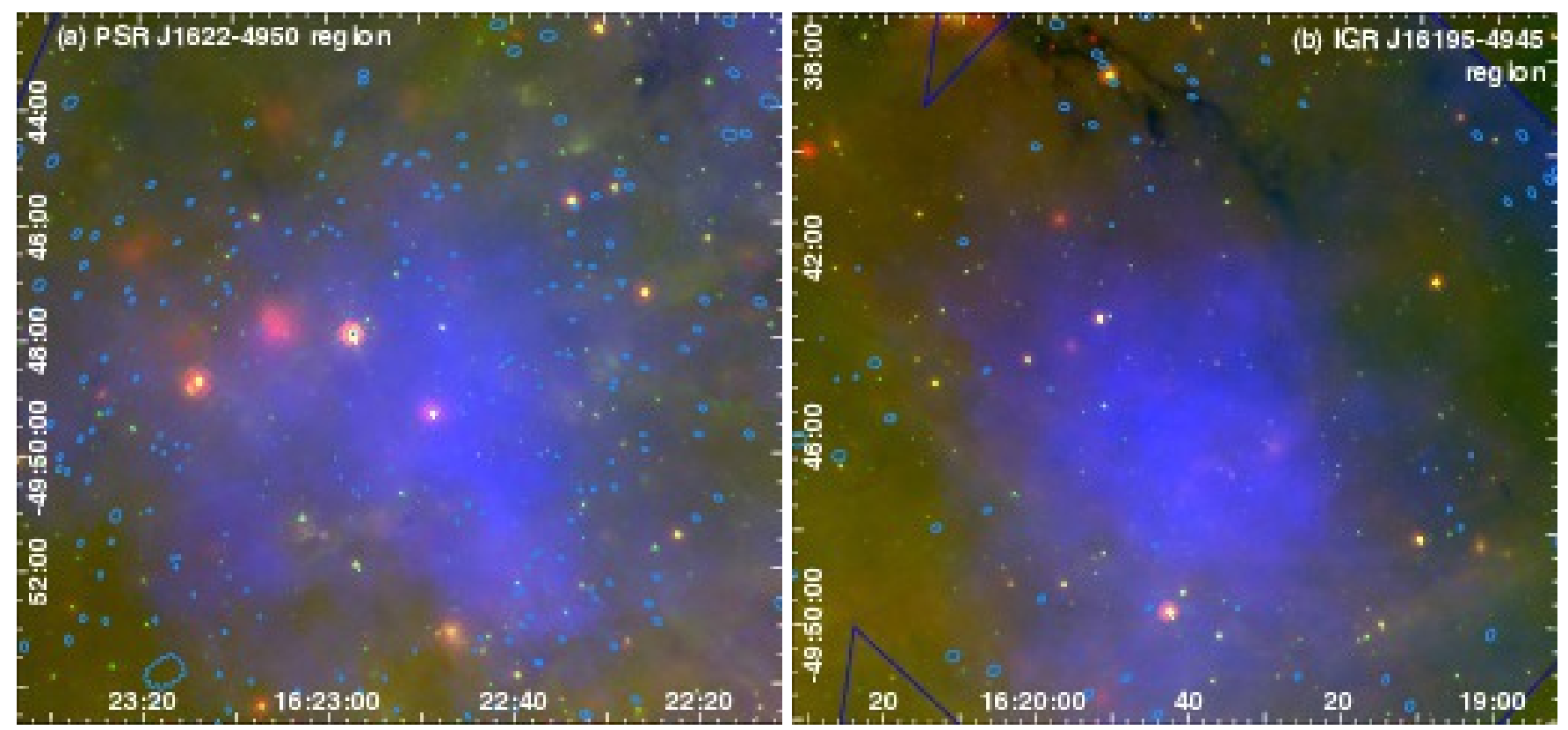

Figure 21. Interesting diffuse X-ray features in archival pointings of the G333 mosaic. (a) Zoomed version of Figure 16(b) for the PSR J1622-4950 pointing, with ACIS point source extraction regions. (b) Zoomed version of Figure 16(b) for the IGR J16195-4945 pointing, with ACIS point source extraction regions.

ObsID 5471 targeted IGR J16195-4945, which is a likely high-mass X-ray binary (Tomsick et al. 2006). This is our source c439 (CXOU J161932.20-494430.6); it is piled up, but there are too few counts to perform pile-up correction. There is bright diffuse X-ray emission in this region (Figure 21(b)). Pandey et al. (2006) note extended radio emission in the vicinity of IGR J16195-4945, so perhaps we are once again seeing X-ray emission from a SNR. 


\subsection{AFGL 2591}

AFGL 2591 is an embedded MYSO with prominent outflows and maser emission, surrounded by a small cluster (Trinidad et al. 2003); it is seen in the direction of the Cygnus X star-forming complex (Reipurth \& Schneider 2008) but it is now believed to be substantially behind Cygnus X (at 3.33 kpc), from parallax of its water masers (Rygl et al. 2012). The radio-bright source VLA-3 provides most of the IR emission and is thought to be a late-O-type MYSO (Sanna et al. 2012).

Parkin et al. (2009) analyzed the Chandra data on AFGL 2591, finding 62 sources on the S3 CCD; our analysis of the same data (Figures 22) yields 152 sources on S3 and 288 sources in the entire observation. In the vicinity of VLA-3, Parkin et al. find 4 X-ray sources but do not detect the MYSO. We recover those 4 X-ray sources and add several more in our analysis (Figure 22(d)), but again we do not detect VLA-3. Parkin et al. interpreted a small excess of counts close to VLA-3 as possible diffuse emission from the winds of the proto-O star. We find a few of those counts to be consistent with a point source east of VLA-3; the remaining small number of counts could come from other point sources (including VLA-3) fainter than our detection limit, from the background, or from other sources of diffuse emission. Firmly establishing diffuse X-ray emission from VLA-3 wind interactions would require much more Chandra data.

Extensive diffuse X-ray emission is seen in this ACIS field, including off-axis on the I2 and I3 CCDs. Figure 22(c) demonstrates that diffuse emission surrounds AFGL 2591; Figure 22(d) shows that faint structures extend down to the embedded core. Some of this diffuse emission could be associated with the foreground Cygnus X complex; future X-ray spectral fitting to infer the absorption across the field may help us locate it along the line of sight. 


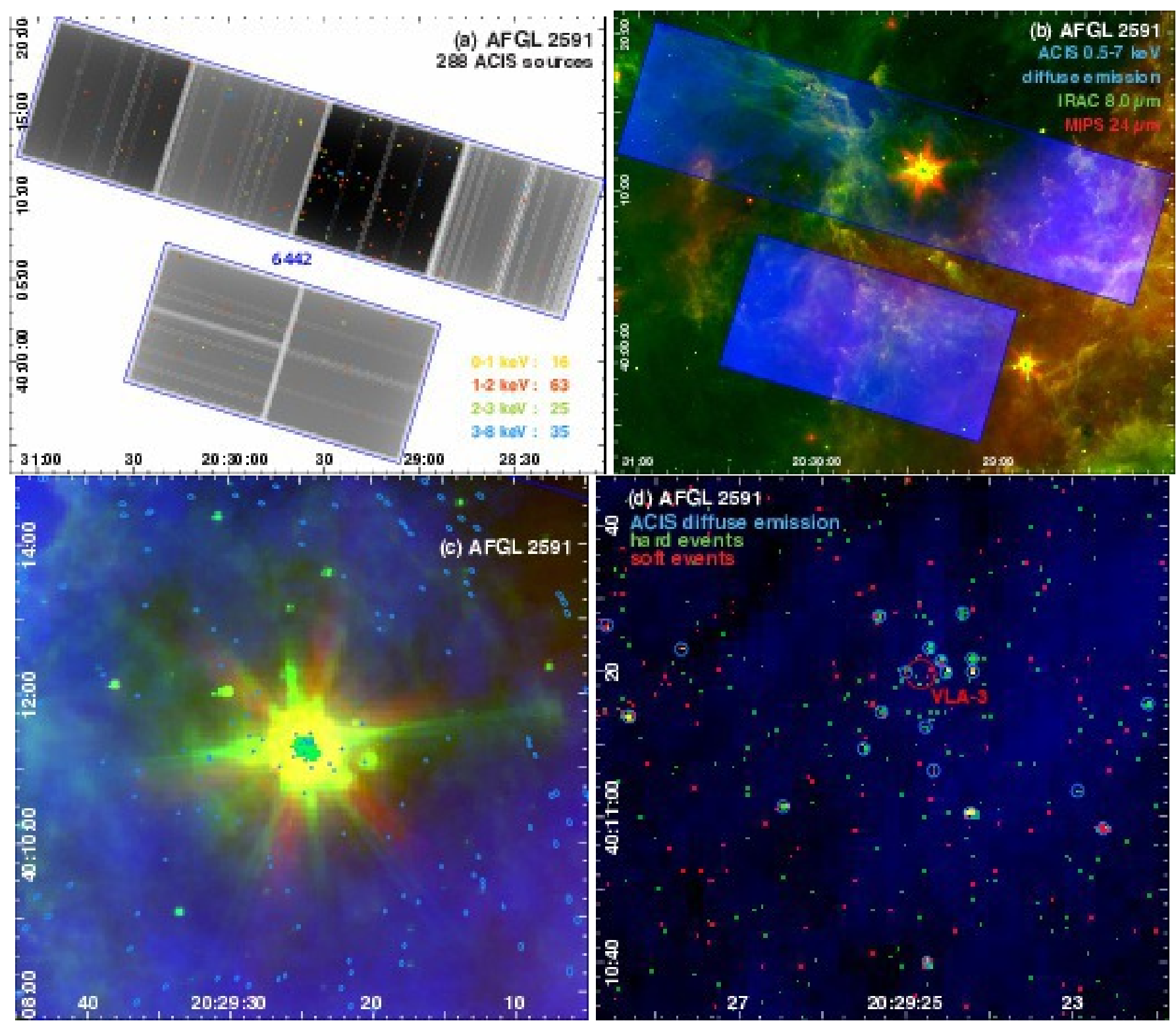

Figure 22. AFGL 2591. (a) ACIS exposure map with brighter ( $\geq 5$ net counts) ACIS point sources overlaid; colors denote median energy for each source. The ObsID number is shown in blue. (b) ACIS diffuse emission in the Spitzer context. (c) Zoom of (b) for the central region around AFGL 2591, with point source extraction regions. (d) ACIS event data and diffuse emission at the core of AFGL 2591. The position of VLA-3 from Sanna et al. (2012) is shown as a red $2^{\prime \prime}$-radius circle, representing the region they searched for masers. 


\subsection{2. $634.4+0.23$}

The first compact IRDC targeted by Chandra was G014.225-00.506 (Povich \& Whitney 2010), part of a large molecular cloud complex associated with the M17 giant Hir region known as the M17 Southwest Extension (Elmegreen et al. 1979), located at a distance of $2.0 \mathrm{kpc}$ (Xu et al. 2011). Using the same analysis methods as those presented here, we catalogued 840 X-ray sources in a 99-ks ACIS-I observation of G014.225-00.506 (Povich et al. 2016). The second IRDC observed by Chandra was the more well-studied object G34.4+0.23 (Rathborne et al. 2005; Shepherd et al. 2007), observed with ACIS-I for just $63 \mathrm{ks}$ under the assumption that it lies at a maser parallax distance of $1.56 \mathrm{kpc}$ (Kurayama et al. 2011), substantially closer than G014.225-00.506. This distance was soon questioned, however (Foster et al. 2012), and recent papers (Foster et al. 2014; Xu et al. 2016) favor the kinematic distance of around $3.7 \mathrm{kpc}$ (Rathborne et al. 2005).

This Chandra observation (Figure 23) reveals few X-ray sources along the G34.4+0.23 IRDC compared to G014.225-00.506 (Povich et al. 2016), again suggesting that the kinematic distance is correct and that the 63-ks ACIS exposure was too short to detect much of the young stellar population embedded in this IRDC. Nevertheless, we do find a large number of X-ray point sources in this field (Figure 23(c)). Some will be foreground and background contaminants, but many hundreds are likely pre-MS stars associated in some way with the star formation going on in this region.

We have X-ray counterparts to a few of the young stellar objects listed in Shepherd et al. (2007). In particular, we find three faint, very hard X-ray sources in close proximity to source \#11 from Shepherd et al., at the center of the UCHıR (Figure 23(d)): c284 (CXOU J185318.71+012449.1, 8 net counts, median energy $4.1 \mathrm{keV}$ ); c283 (CXOU J185318.71+012446.3, 6 net counts, median energy $4.5 \mathrm{keV}$ ); cc554 (CXOU J185318.54+012445.2, 4 net counts, median energy $4.2 \mathrm{keV}$ ).

Foster et al. (2014) found a distributed population of low-mass protostars associated with the IRDC but located outside the densest parts of the filament. Perhaps our X-ray sources sample an even older and more distributed stellar population. Further multiwavelength analysis is necessary to understand this X-ray source population and the history of star formation across this region.

The G34.4+0.23 Chandra mosaic shows abundant diffuse X-ray emission. A small patch at field center may contain unresolved point sources from the central proto-cluster (Figure 23(d)). This is largely surrounded by dark regions, perhaps due to shadowing by the IRDC and surrounding molecular material. Diffuse emission is seen again at the field edges, especially at the east and west corners of the ACIS-I image. As often demonstrated in other MOXC2 targets, the off-axis ACIS-S CCDs hint at more diffuse X-ray structures far off-axis. This emission is anticoincident with moderately bright Spitzer $8 \mu \mathrm{m}$ emission to the southeast of G34.4+0.23.

The more developed MSFR G34.26+0.15 was partially imaged off-axis in this observation, at the southern corner of the ACIS-I array (Figure 23(b)). A few X-ray point sources are found there (likely limited by low sensitivity and source confusion) and the region is surrounded by diffuse X-ray emission. A Chandra observation centered on this MSFR would probably reveal a rich young stellar population and an interesting network of hot plasma from massive star feedback in G34.26+0.15. 

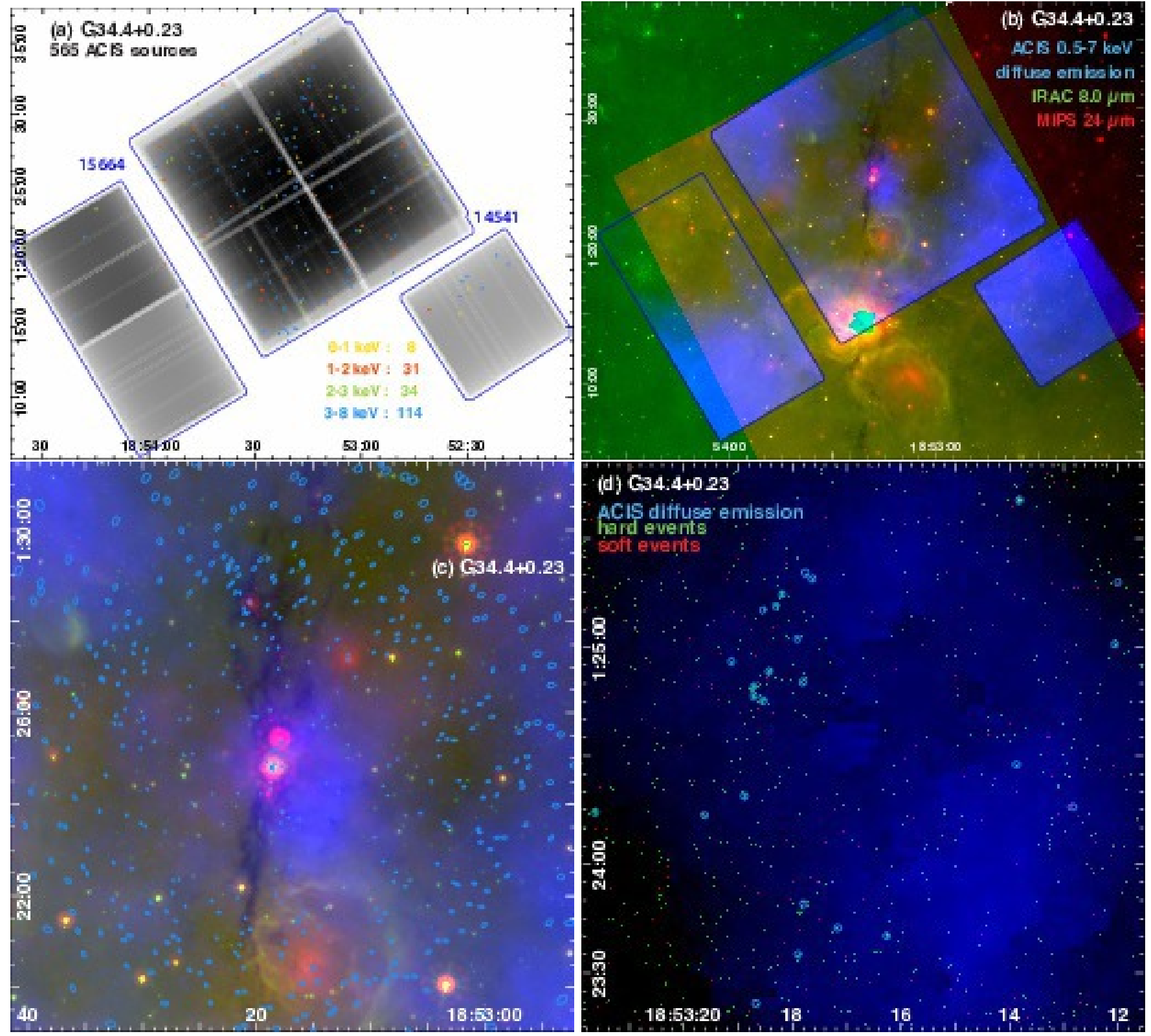

Figure 23. G34.4+0.23. (a) ACIS exposure map with brighter ( $\geq 5$ net counts) ACIS point sources overlaid; colors denote median energy for each source. ObsID numbers are shown in blue. (b) ACIS diffuse emission in the Spitzer context. (c) Zoom of (b) centered on G34.4+0.23, with point source extraction regions. (d) ACIS event data and diffuse emission at the center of G34.4+0.23. 


\subsection{Westerlund 1}

We finish our tour of MOXC2 MSFRs with Westerlund 1 (Wd1), the most massive YMC known in the Galaxy (Andersen et al. 2017). It is a monolithic cluster, not part of a complex of multiple MSFRs, as we have seen in many MOXC2 targets. The center of Wd1 is known to be elongated (Gennaro et al. 2011), but recent work (Gennaro et al. 2017) finds that its massive stars are not strongly concentrated towards the center (mass-segregated); these authors conclude that Wd1 has changed little in size or density since its formation. Its combination of mass and age explains its uniquely large population of evolved massive stars, including red supergiants, yellow hypergiants, and WRs; Wd1 may have weathered 100 supernovae already (Clark et al. 2008). Twelve WRs (Skinner et al. 2006) and a magnetar (Muno et al. 2006) were among the 241 point sources detected in the original 57-ks ACIS-S observation (Clark et al. 2008).

We recover all but six of the X-ray sources tabulated in Clark et al. (2008). We find many hundreds more X-ray sources and diffuse emission across our ACIS mosaic (Figure 24). The magnetar (CXOU J164710.2-455216) suffers from photon pile-up in these observations. Clark et al. (2008) presented X-ray spectral fits for the brightest ACIS sources, so we will not report further spectral fitting results here.

Wd1 is four times as massive as NGC 3603 (featured in MOXC1) but eight times lower in central density (Figer 2008). This and the relatively short Chandra exposure on Wd1 means that our X-ray point source list suffers much less confusion than we found in NGC 3603 (MOXC1). After excising resolved point sources, the ACIS event data still show a substantial overdensity of counts at the center of Wd1 (Figure 24(c)); a longer Chandra observation would undoubtedly resolve out a large number of additional X-ray point sources in the cluster core.

The second pointing, to the south of Wd1 in our ACIS mosaic (ObsID 11836, $10 \mathrm{ks}$ ), is an ACIS-I GTO observation of 0FGL J1648.1-4606, part of a snapshot survey of unidentified Fermi/LAT GeV sources. PSR J1648-4611 in this field is listed as a non-detection in Table 2 of Kargaltsev et al. (2012). This source remains undetected in our analysis.

Diffuse X-ray emission from Wd1 was studied by Muno et al. (2006) (Chandra) and Kavanagh et al. (2011) (XMM). In our ACIS mosaic (Figure 24(b)), we see diffuse emission across much of the field, including the cluster center (Figure 24(c)). The unresolved X-ray emission at the northwest corner of the mosaic is due in large part to the bright LMXB GX340+0, located off the field. We have masked the brightest of this scattered light from our diffuse images. Northeast of Wd1, far off-axis on the S0 chip of ObsID 6283, we have another serendipitous detection of diffuse X-ray emission apparently associated with a bubble structure in the Spitzer data (Figure 24(d)). 


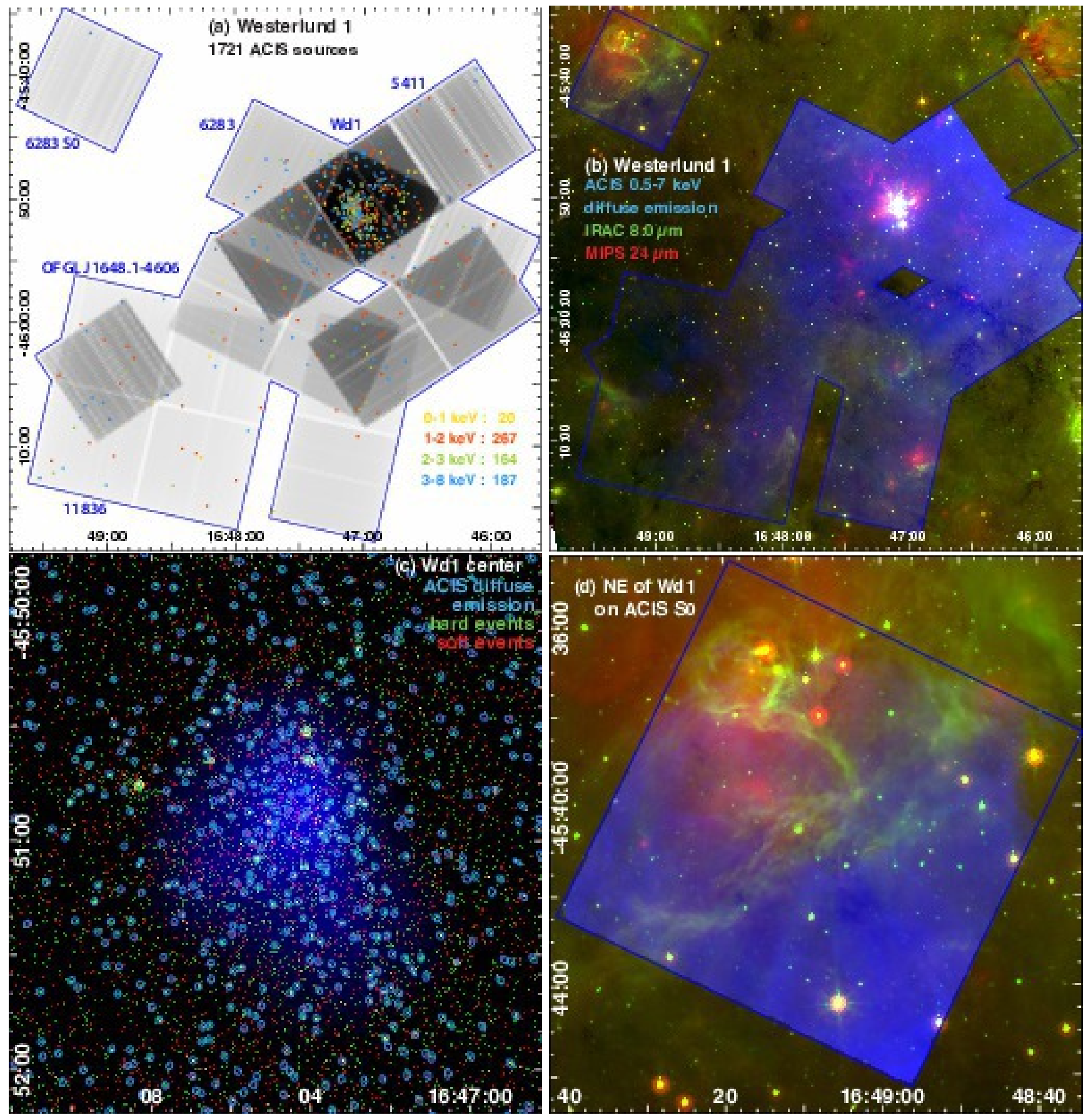

Figure 24. Wd1. (a) ACIS exposure map with brighter ( $\geq 5$ net counts) ACIS point sources overlaid; colors denote median energy for each source. ObsID numbers and regions named in the text are shown in blue. (b) ACIS diffuse emission in the Spitzer context. (c) ACIS event data and diffuse emission on the Wd1 cluster core. (d) Zoomed version of (b) for the far off-axis S0 chip in the 19-ks ObsID 6283. 


\section{SUMMARY}

Expanding on the results presented in MOXC1 (Townsley et al. 2014), with MOXC2 we have assembled and analyzed 13 Chandra/ACIS Galactic Plane mosaics featuring 16 MSFRs. These mosaics combine Chandra archival, GO, and GTO datasets to form the most complete high-resolution X-ray picture possible of the MSFRs and their surrounding environments. Major software and procedural changes for MOXC2 have made it possible to include far off-axis data from ACIS-I observations and archival data using the ACIS-S imaging configuration. This broadens the fields covered by our mosaics and the MSFRs we can access; most importantly, these changes reveal the ubiquity of hot plasmas pervading the Plane and Chandra's amazing ability to trace their diffuse X-ray emission.

We present properties for more than 18,000 X-ray point sources found in these fields. From past experience, most of these sources will turn out to be pre-MS MSFR members; Chandra will increase the census of young stars in every MOXC2 MSFR. We find likely CWBs, IMPS, and the ionizing sources of well-known radio UCHıRs; these sources are rare and stand out because of their extremes in X-ray luminosity, variability, and hard spectra. They demonstrate that X-ray emission turns on early in the star formation process, at least in some cases, bombarding cold molecular environments with hard radiation and shocks.

We excised the X-ray point sources from each mosaic and made adaptively-smoothed images from the remaining X-ray emission. We find that this unresolved emission appears to be truly diffuse in many cases, tracing hot plasma in and around these MSFRs. This hot plasma appears to pervade cluster centers, fill Spitzer bubbles, and leak out of perforated Hı region boundaries. Although MOXC2 MSFRs display a wide variety of diffuse X-ray morphologies and surface brightnesses, none lacks diffuse X-ray emission. With detailed spectral studies, pressures and densities of these hot plasmas will give physical insights into the birth of the hot ISM.

We hope that our X-ray point source catalogs will be useful to the wider star formation community for understanding young stellar populations in and around MSFRs. The next step is to match these $\mathrm{X}$-ray source lists to longer-wavelength datasets in order to deduce which X-ray sources are cluster members and, in turn, to study multiwavelength properties of those cluster members. For diffuse X-ray emission, bright fields need to be tessellated, spectra from each tessellate extracted and fitted, and maps generated of spectral fit parameters (Townsley et al. 2011b). Even for faint fields, a rough spectral characterization should be possible for the diffuse emission pervading every clump and cluster of young stars in MOXC2.

The Chandra Cycle 18 archival program MSFRs Across the Galaxy in X-rays (MAGiX) is underway; it will provide ACIS source lists and map diffuse X-ray emission for another 16 Galactic MSFRs. MAGiX2 is a new Chandra Cycle 19 archival program that will perform similar ACIS data analysis on a further 13 Galactic MSFRs plus three more in the Magellanic Clouds. With these and other ongoing Chandra programs, by the end of the decade we will add 40 MSFRs analyzed with similar methods to the body of work amassed already by COUP, CCCP, MYStIX, MOXC1, SFiNCs, MOXC2, and other Chandra projects. We hope that these efforts demonstrate the benefit of Chandra observations to MSFR studies and facilitate the use of Chandra data by the multiwavelength star formation community.

Finally, MOXC2 shows that there are still many important observations for Chandra to make on MSFRs. Coverage is shallow or absent for a surprising number of famous MSFRs that are mainstays of longer-wavelength star formation science. We need to explore younger regions (IRDCs) and 
older regions (red supergiant clusters), rich MSFR complexes near (G352) and far (W31), molecular filaments anchoring the spiral arms (Nessie) and cauldrons of massive star evolution (Wd1). Much discovery awaits us.

Acknowledgments: We appreciate the time and effort donated by our anonymous referee to comment on this paper. This work was supported by Chandra X-ray Observatory general observer grants GO314002X, GO5-16015X, and GO6-17132X (PI: L. Townsley), and GO9-0155X (PI: Bryan Gaensler), and by the Penn State ACIS Instrument Team Contract SV4-74018. All of these were issued by the Chandra X-ray Center, which is operated by the Smithsonian Astrophysical Observatory for and on behalf of NASA under contract NAS8-03060. The ACIS Guaranteed Time Observations included here were selected by the ACIS Instrument Principal Investigator, Gordon P. Garmire, of the Huntingdon Institute for X-ray Astronomy, LLC, which is under contract to the Smithsonian Astrophysical Observatory; Contract SV2-82024. M.S.P. is supported by the National Science Foundation through grant CAREER-1454333. T.N. was supported for the final part of this work through a Leverhulme Trust Research Project Grant. This research used data products from the Chandra Data Archive, software provided by the Chandra X-ray Center in the application package CIAO, and SAOImage $D S 9$ software developed by the Smithsonian Astrophysical Observatory. This research also used data products from the Spitzer Space Telescope, operated by the Jet Propulsion Laboratory (California Institute of Technology) (JPL/CalTech) under a contract with NASA, and data products from the Wide-field Infrared Survey Explorer (WISE), which is a joint project of the University of California, Los Angeles and JPL/CalTech, funded by NASA. This research used NASA's Astrophysics Data System Bibliographic Services, and the SIMBAD database and VizieR catalog access tool provided by CDS, Strasbourg, France.

Facilities: CXO (ACIS), Spitzer (IRAC, MIPS), WISE.

Software: ACIS Extract (Broos et al. 2010, 2012; Broos \& Townsley 2016), CIAO (Fruscione et al. 2006), DS9 (Joye \& Mandel 2003), MARX (Davis et al. 2012). 


\section{REFERENCES}

Aharonian, F., Akhperjanian, A. G., Barres de Almeida, U., et al. 2008, A\&A, 486, 829

Andersen, M., Gennaro, M., Brandner, W., et al. 2017, A\&A, 602, A22

Anderson, C. N., Hofner, P., Shepherd, D., \& Creech-Eakman, M. 2011, AJ, 142, 158

Anderson, G. E., Gaensler, B. M., Slane, P. O., et al. 2012, ApJ, 751, 53

Anderson, G. E., Gaensler, B. M., Kaplan, D. L., et al. 2014, ApJS, 212, 13

Anderson, L. D., Deharveng, L., Zavagno, A., et al. 2015, ApJ, 800, 101

André, P., Di Francesco, J., Ward-Thompson, D., et al. 2014, Protostars and Planets VI, 27

Arias, J. I., Walborn, N. R., Simón Díaz, S., et al. 2016, AJ, 152, 31

Arnaud, K. A. 1996, in ASP Conf. Ser. 101, Astronomical Data Analysis Software \& Systems V, ed. G. H. Jacoby \& J. Barnes (San Francisco, CA: ASP), 17

Babel, J., \& Montmerle, T. 1997, A\&A, 323, 121

Balog, Z., Kenyon, S. J., Lada, E. A., et al. 2004, AJ, 128, 2942

Bastian, N., Ercolano, B., Gieles, M., et al. 2007, MNRAS, 379, 1302

Beck, S. C., Kelly, D. M., \& Lacy, J. H. 1998, AJ, 115,2504

Becker, R. H., \& Helfand, D. J. 1988, AJ, 95, 883

Beuther, H., Kerp, J., Preibisch, T., Stanke, T., \& Schilke, P. 2002, A\&A, 395, 169

Beuther, H., Schilke, P., Menten, K. M., et al. 2002, ApJ, 566, 945

Beuther, H., Walsh, A., Schilke, P., et al. 2002, A\&A, 390, 289

Beuther, H., Schilke, P., \& Stanke, T. 2003, A\&A, 408, 601

Beuther, H., Linz, H., Henning, T., Feng, S., \& Teague, R. 2017, A\&A, 605, A61

Bik, A., Kaper, L., Hanson, M. M., \& Smits, M. 2005, A\&A, 440, 121

Bik, A., Kaper, L., \& Waters, L. B. F. M. 2006, A\&A, 455, 561

Blum, R. D., Conti, P. S., \& Damineli, A. 2000, AJ, 119, 1860

Blum, R. D., Damineli, A., \& Conti, P. S. 2001, AJ, 121, 3149

Breen, S. L., Ellingsen, S. P., Johnston-Hollitt, M., et al. 2007, MNRAS, 377, 491
Brogan, C. L., Gelfand, J. D., Gaensler, B. M., Kassim, N. E., \& Lazio, T. J. W. 2006, ApJL, 639, L25

Broos, P. S., Townsley, L. K., Feigelson, E. D., et al. 2010, ApJ, 714, 1582

Broos, P. S., Townsley, L. K., Feigelson, E. D., et al. 2011, ApJS, 194, 2

Broos, P. S., Townsley, L. K., Getman, K. V., Bauer, F. E. 2012, Astrophysics Source Code Library, 1203.001

Broos, P. S., Getman, K. V., Povich, M. S., et al. 2013, ApJS, 209, 32

Broos, P. S., \& Townsley, L. K. 2016, ACIS Extract software package, Zenodo, https://doi.org/10.5281/zenodo.781433

Cesaroni, R., Neri, R., Olmi, L., et al. 2005, A\&A, 434, 1039

Cesaroni, R., Galli, D., Neri, R., \& Walmsley, C. M. 2014, A\&A, 566, A73

Chandrasekhar, S., \& Fermi, E. 1953, ApJ, 118, 116

Chibueze, J. O., Omodaka, T., Handa, T., et al. 2014, ApJ, 784, 114

Churchwell, E., Povich, M. S., Allen, D., et al. 2006, ApJ, 649, 759

Churchwell, E., Watson, D. F., Povich, M. S., et al. 2007, ApJ, 670, 428

Clark, J. S., Muno, M. P., Negueruela, I., et al. 2008, A\&A, 477, 147

Clark, J. S., Ritchie, B. W., \& Negueruela, I. 2013, A\&A, 560, A11

Conti, P. S., \& Crowther, P. A. 2004, MNRAS, 355,899

Davies, B., Figer, D. F., Law, C. J., et al. 2008, ApJ, 676, 1016

Davies, B., Lumsden, S. L., Hoare, M. G., Oudmaijer, R. D., \& de Wit, W.-J. 2010, MNRAS, 402, 1504

Davis, C. J., Kumar, M. S. N., Sandell, G., et al. 2007, MNRAS, 374, 29

Davis, J. E., Bautz, M. W., Dewey, D., et al. 2012, Proc. SPIE, 8443, 84431A

De Buizer, J. M., Liu, M., Tan, J. C., et al. 2017, ApJ, 843, 33

Deharveng, L., \& Zavagno, A. 2008, Handbook of Star Forming Regions, Volume II, 5, 437

Deharveng, L., Zavagno, A., Schuller, F., et al. 2009, A\&A, 496, 177 
Deharveng, L., Schuller, F., Anderson, L. D., et al. 2010, A\&A, 523, A6

Deharveng, L., Zavagno, A., Samal, M. R., et al. 2015, A\&A, 582, A1

Dewangan, L. K., Luna, A., Ojha, D. K., et al. 2015, ApJ, 811, 79

Dewangan, L. K., Ojha, D. K., Grave, J. M. C., \& Mallick, K. K. 2015, MNRAS, 446, 2640

Dubner, G. M., Moffett, D. A., Goss, W. M., \& Winkler, P. F. 1993, AJ, 105, 2251

Dyck, H. M., \& Simon, T. 1977, ApJ, 211, 421

Elmegreen, B. G., \& Lada, C. J. 1977, ApJ, 214, 725

Elmegreen, B. G., Lada, C. J., \& Dickinson, D. F. 1979, ApJ, 230, 415

Elmegreen, B. G. 2008, ApJ, 672, 1006

Evans, I. N., Primini, F. A., Glotfelty, K. J., et al. 2010, ApJS, 189, 37

Ezoe, Y., Kokubun, M., Makishima, K., Sekimoto, Y., \& Matsuzaki, K. 2006, ApJ, 638, 860

Feigelson, E. D., Martin, A. L., McNeill, C. J., Broos, P. S., \& Garmire, G. P. 2009, AJ, 138, 227

Feigelson, E. D., Townsley, L. K., Broos, P. S., et al. 2013, ApJS, 209, 26

Figer, D. F., MacKenty, J. W., Robberto, M., et al. 2006, ApJ, 643, 1166

Figer, D. F. 2008, "Massive Stars as Cosmic Engines," IAU Symp. 250, 247

Figueira, M., Zavagno, A., Deharveng, L., et al. 2017, A\&A, 600, A93

Figuerêdo, E., Blum, R. D., Damineli, A., \& Conti, P. S. 2005, AJ, 129, 1523

Foster, J. B., Stead, J. J., Benjamin, R. A., Hoare, M. G., \& Jackson, J. M. 2012, ApJ, 751, 157

Foster, J. B., Arce, H. G., Kassis, M., et al. 2014, ApJ, 791, 108

Frank, K. A., Burrows, D. N., \& Park, S. 2015, ApJ, 810, 113

Frau, P., Girart, J. M., Zhang, Q., \& Rao, R. 2014, A\&A, 567, A116

Froebrich, D., \& Scholz, A. 2013, MNRAS, 436, 1116

Fruscione, A., et al. 2006, Proc. SPIE, 6270

Funk, S., Hinton, J. A., Moriguchi, Y., et al. 2007, A\&A, 470, 249

Furness, J. P., Crowther, P. A., Morris, P. W., et al. 2010, MNRAS, 403, 1433

Gagné, M., Fehon, G., Savoy, M. R., et al. 2011, ApJS, 194, 5
Galván-Madrid, R., Zhang, Q., Keto, E., et al. 2010, ApJ, 725, 17

Garmire, G. P., Bautz, M. W., Ford, P. G., Nousek, J. A., \& Ricker, G. R., Jr. 2003, Proc. SPIE, 4851, 28

Gennaro, M., Brandner, W., Stolte, A., \&

Henning, T. 2011, MNRAS, 412, 2469

Gennaro, M., Goodwin, S. P., Parker, R. J., Allison, R. J., \& Brandner, W. 2017, MNRAS, 472,1760

Getman, K. V., Flaccomio, E., Broos, P. S., et al. 2005, ApJS, 160, 319

Getman, K. V., Feigelson, E. D., Broos, P. S., Townsley, L. K., \& Garmire, G. P. 2010, ApJ, 708,1760

Getman, K. V., Broos, P. S., Kuhn, M. A., et al. 2017, ApJS, 229, 28

Ghosh, S. K., Iyengar, K. V. K., Rengarajan, T. N., et al. 1989, ApJ, 347, 338

Goodman, A. A., Alves, J., Beaumont, C. N., et al. 2014, ApJ, 797, 53

Gotthelf, E. V., \& Halpern, J. P. 2008, ApJ, 681, 515

Gotthelf, E. V., \& Halpern, J. P. 2009, ApJL, 700, L158

Gregory, S. G., Adams, F. C., \& Davies, C. L. 2016, MNRAS, 457, 3836

Güdel, M., \& Nazé, Y. 2009, A\&A Rv, 17, 309

Helfand, D. J., Gotthelf, E. V., Halpern, J. P., et al. 2007, ApJ, 665, 1297

Hofner, P., Cesaroni, R., Olmi, L., et al. 2007, A\&A, 465, 197

Immer, K., Reid, M. J., Menten, K. M., Brunthaler, A., \& Dame, T. M. 2013, A\&A, 553, A117

Immer, K., Galván-Madrid, R., König, C., Liu, H. B., \& Menten, K. M. 2014, A\&A, 572, A63

Jackson, J. M., Finn, S. C., Chambers, E. T., Rathborne, J. M., \& Simon, R. 2010, ApJL, 719, L185

Joye, W. A., \& Mandel, E. 2003, Astronomical Data Analysis Software and Systems XII, 295, 489

Kainulainen, J., Alves, J., Beuther, H., Henning, T., \& Schuller, F. 2011, A\&A, 536, A48

Kaplan, D. L., Fox, D. W., Kulkarni, S. R., et al. 2002, ApJ, 564, 935

Kargaltsev, O., Durant, M., Pavlov, G. G., \& Garmire, G. 2012, ApJS, 201, 37 
Kavanagh, P. J., Norci, L., \& Meurs, E. J. A. 2011, NewA, 16, 461

Kharchenko, N. V., Piskunov, A. E., Röser, S., Schilbach, E., \& Scholz, R.-D. 2005, A\&A, 438, 1163

Kuhn, M. A., Getman, K. V., Broos, P. S., Townsley, L. K., \& Feigelson, E. D. 2013, ApJS, 209, 27

Kumar, M. S. N., Davis, C. J., Grave, J. M. C., Ferreira, B., \& Froebrich, D. 2007, MNRAS, 374,54

Kurayama, T., Nakagawa, A., Sawada-Satoh, S., et al. 2011, PASJ, 63, 513

Levin, L., Bailes, M., Bates, S., et al. 2010, ApJL, 721, L33

Lo, N., Wiles, B., Redman, M. P., et al. 2015, MNRAS, 453, 3245

Luisi, M., Anderson, L. D., Balser, D. S., Bania, T. M., \& Wenger, T. V. 2016, ApJ, 824, 125

Maíz Apellániz, J., Sota, A., Arias, J. I., et al. 2016, ApJS, 224, 4

Mallick, K. K., Ojha, D. K., Tamura, M., et al. 2014, MNRAS, 443, 3218

Massey, P., Johnson, K. E., \& Degioia-Eastwood, K. 1995, ApJ, 454, 151

McCaughrean, M., Rayner, J., \& Zinnecker, H. 1991, Mem. Soc. Astron. Italiana, 62, 715

Mercer, E. P., Clemens, D. P., Meade, M. R., et al. 2005, ApJ, 635, 560

Messineo, M., Figer, D. F., Davies, B., et al. 2008, ApJL, 683, L155

Messineo, M., Davies, B., Figer, D. F., et al. 2011, ApJ, 733, 41

Messineo, M., Clark, J. S., Figer, D. F., et al. 2015, ApJ, 805, 110

Montes, V. A., Hofner, P., Anderson, C., \& Rosero, V. 2015, ApJS, 219, 41

Mookerjea, B., Kramer, C., Nielbock, M., \& Nyman, L.-A. 2004, A\&A, 426, 119

Moscadelli, L., Reid, M. J., Menten, K. M., et al. 2009, ApJ, 693, 406

Moscadelli, L., Cesaroni, R., Rioja, M. J., Dodson, R., \& Reid, M. J. 2011, A\&A, 526, A66

Motte, F., Bontemps, S., \& Louvet, F. 2017, arXiv:1706.00118

Muno, M. P., Clark, J. S., Crowther, P. A., et al. 2006, ApJL, 636, L41

Muno, M. P., Law, C., Clark, J. S., et al. 2006, ApJ, 650, 203
Nazé, Y., Broos, P. S., Oskinova, L., et al. 2011, ApJS, 194, 7

Nebot Gómez-Morán, A., Motch, C., Pineau, F.-X., et al. 2015, MNRAS, 452, 884

Nguyên, H., Nguyên Lu'o'ng, Q., Martin, P. G., et al. 2015, ApJ, 812, 7

Ochsenbein, F., Bauer, P., \& Marcout, J. 2000, A\&AS, 143, 23

Ojha, D. K., Tamura, M., Nakajima, Y., et al. 2004, ApJ, 616, 1042

Pandey, M., Rao, A. P., Manchanda, R., Durouchoux, P., \& Ishwara-Chandra, C. H. 2006, A\&A, 453, 83

Pannuti, T. G., Rho, J., Heinke, C. O., \& Moffitt, W. P. 2014, AJ, 147, 55

Parkin, E. R., Pittard, J. M., Hoare, M. G., Wright, N. J., \& Drake, J. J. 2009, MNRAS, 400, 629

Persi, P., Tapia, M., \& Smith, H. A. 2006, A\&A, 445, 971

Persi, P., \& Tapia, M. 2008, Handbook of Star Forming Regions, Volume II, 5, 456

Portegies Zwart, S. F., McMillan, S. L. W., \& Gieles, M. 2010, ARA\&A, 48, 431

Povich, M. S., \& Whitney, B. A. 2010, ApJL, 714, L285

Povich, M. S., Smith, N., Majewski, S. R., et al. 2011, ApJS, 194, 14

Povich, M. S., Townsley, L. K., Robitaille, T. P., et al. 2016, ApJ, 825, 125

Prato, L., Rice, E. L., \& Dame, T. M. 2008, Handbook of Star Forming Regions, Volume I, 4, 18

Preibisch, T., Kim, Y.-C., Favata, F., et al. 2005, ApJS, 160, 401

Puga, E., Marín-Franch, A., Najarro, F., et al. 2010, A\&A, 517, A2

Rathborne, J. M., Jackson, J. M., Chambers, E. T., et al. 2005, ApJL, 630, L181

Rathborne, J. M., Jackson, J. M., \& Simon, R. 2006, ApJ, 641, 389

Rauw, G., \& Nazé, Y. 2016, Advances in Space Research, 58, 761

Reipurth, B., \& Schneider, N. 2008, Handbook of Star Forming Regions, Volume I, 4, 36

Riaz, B., Martín, E. L., Tata, R., et al. 2012, MNRAS, 419, 1887

Richards, E. E., Lang, C. C., Trombley, C., \& Figer, D. F. 2012, AJ, 144, 89 
Rodón, J. A., Beuther, H., \& Schilke, P. 2012, A\&A, 545, A51

Roman-Lopes, A., Abraham, Z., \& Lépine, J. R. D. 2003, AJ, 126, 1896

Roman-Lopes, A., Abraham, Z., Ortiz, R., \& Rodriguez-Ardila, A. 2009, MNRAS, 394, 467

Russeil, D., Adami, C., Amram, P., et al. 2005, A\&A, 429, 497

Russeil, D., Zavagno, A., Adami, C., et al. 2012, A\&A, 538, A142

Russeil, D., Schneider, N., Anderson, L. D., et al. 2013, A\&A, 554, A42

Russeil, D., Tigé, J., Adami, C., et al. 2016, A\&A, 587, A135

Rygl, K. L. J., Brunthaler, A., Sanna, A., et al. 2012, A\&A, 539, A79

Sana, H., Le Bouquin, J.-B., Lacour, S., et al. 2014, ApJS, 215, 15

Sandell, G., Goss, W. M., \& Wright, M. 2005, ApJ, 621, 839

Sanna, A., Reid, M. J., Carrasco-González, C., et al. 2012, ApJ, 745, 191

Sharma, S., Pandey, A. K., Ojha, D. K., et al. 2017, MNRAS, 467, 2943

Shepherd, D. S., Testi, L., \& Stark, D. P. 2003, ApJ, 584, 882

Shepherd, D. S., Kurtz, S. E., \& Testi, L. 2004, ApJ, 601, 952

Shepherd, D. S., Povich, M. S., Whitney, B. A., et al. 2007, ApJ, 669, 464

Shi, H. M., \& Hu, J. Y. 1999, A\&AS, 136, 313

Skinner, S. L., Simmons, A. E., Zhekov, S. A., et al. 2006, ApJL, 639, L35

Skrutskie, M. F., Cutri, R. M., Stiening, R., et al. 2006, AJ, 131, 1163

Sota, A., Maíz Apellániz, J., Morrell, N. I., et al. 2014, ApJS, 211, 10

Tackenberg, J., Beuther, H., Henning, T., et al. 2014, A\&A, 565, A101

Tapia, M., \& Persi, P. 2008, Handbook of Star Forming Regions, Volume II, 5, 449
Tapia, M., Rodríguez, L. F., Persi, P., Roth, M., \& Gómez, M. 2009, AJ, 137, 4127

Tigé, J., Motte, F., Russeil, D., et al. 2017, A\&A, 602, A77

Tomsick, J. A., Chaty, S., Rodriguez, J., et al. 2006, ApJ, 647, 1309

Townsley, L. K., Feigelson, E. D., Montmerle, T., et al. 2003, ApJ, 593, 874

Townsley, L. K., Broos, P. S., Corcoran, M. F., et al. 2011, ApJS, 194, 1

Townsley, L. K., Broos, P. S., Chu, Y.-H., et al. 2011, ApJS, 194, 15

Townsley, L. K., Broos, P. S., Garmire, G. P., et al. 2014, ApJS, 213, 1 (MOXC1)

Townsley, L. K., \& Broos, P. S. 2017, MOXC2 data products, Zenodo, https://doi.org/10.5281/zenodo.1067749

Trinidad, M. A., Curiel, S., Cantó, J., et al. 2003, ApJ, 589, 386

Urquhart, J. S., Thompson, M. A., Moore, T. J. T., et al. 2013, MNRAS, 435, 400

Watson, C., Corn, T., Churchwell, E. B., et al. 2009, ApJ, 694, 546

Weisskopf, M. C., Wu, K., Trimble, V., et al. 2007, ApJ, 657, 1026

Willis, S., Marengo, M., Allen, L., et al. 2013, ApJ, 778, 96

Wright, N. J., Drake, J. J., Guarcello, M. G., et al. 2014, arXiv:1408.6579

Wu, Y. W., Sato, M., Reid, M. J., et al. 2014, A\&A, 566, A17

Xu, J.-L., \& Wang, J.-J. 2012, A\&A, 543, A24

Xu, J.-L., Li, D., Zhang, C.-P., et al. 2016, ApJ, 819, 117

Xu, Y., Reid, M. J., Menten, K. M., et al. 2009, ApJ, 693, 413

Xu, Y., Moscadelli, L., Reid, M. J., et al. 2011, ApJ, 733, 25

Zavagno, A., Pomarès, M., Deharveng, L., et al. 2007, A\&A, 472, 835

Zavagno, A., Russeil, D., Motte, F., et al. 2010, A\&A, 518, L81 